\title{
WestVirginiaUniversity
}

THE RESEARCH REPOSITORY @ WVU

Graduate Theses, Dissertations, and Problem Reports

2017

\section{A Study of the Fast Radio Burst Population}

\author{
Akshaya Rane
}

Follow this and additional works at: https://researchrepository.wvu.edu/etd

\section{Recommended Citation}

Rane, Akshaya, "A Study of the Fast Radio Burst Population" (2017). Graduate Theses, Dissertations, and Problem Reports. 7120.

https://researchrepository.wvu.edu/etd/7120

This Dissertation is protected by copyright and/or related rights. It has been brought to you by the The Research Repository @ WVU with permission from the rights-holder(s). You are free to use this Dissertation in any way that is permitted by the copyright and related rights legislation that applies to your use. For other uses you must obtain permission from the rights-holder(s) directly, unless additional rights are indicated by a Creative Commons license in the record and/ or on the work itself. This Dissertation has been accepted for inclusion in WVU Graduate Theses, Dissertations, and Problem Reports collection by an authorized administrator of The Research Repository @ WVU.

For more information, please contact researchrepository@mail.wvu.edu. 


\title{
A study of the Fast Radio Burst population
}

\author{
Akshaya Rane \\ Dissertation submitted to the \\ Eberly College of Arts and Sciences \\ at West Virginia University \\ in partial fulfillment of the requirements for the degree of \\ Doctor of Philosophy \\ in \\ Physics \\ Duncan Lorimer, Ph.D., Chair \\ Maura McLaughlin, Ph.D. \\ D. J. Pisano, Ph.D. \\ Paul Cassak, Ph.D. \\ Kevin Bandura, Ph.D. \\ Department of Physics and Astronomy \\ Morgantown, West Virginia \\ 2017
}

Keywords: Radio transients, population synthesis

Copyright 2017 Akshaya Rane 


\title{
ABSTRACT \\ A study of the Fast Radio Burst population
}

\author{
Akshaya Rane
}

Fast Radio Bursts (FRBs) are a new class of astrophysical phenomena discovered a decade ago that are characterized by extremely bright millisecond duration radio bursts originating in external galaxies. We have processed archival pulsar survey data obtained with the 64-m Parkes radio telescope in Australia. We used the single-pulse search method to search for pulsars, rotating radio transients (RRATs), and FRBs. RRATs are Galactic pulsars that emit sporadic pulses. We detected 20 known pulsars and one RRAT in this analysis but we did not find any FRBs. We combined this null result with the previous searches carried out with Parkes to constrain an all-sky event rate of FRBs. We used a total of ten surveys and found an event rate of $R=3.3_{-2.2}^{+3.7} \times 10^{3}$ events per day per sky above a fluence (observed flux measured in Jansky $\times$ observed width of the pulse in milliseconds) limit of 4.0 Jy ms at the $99 \%$ confidence level.

We also carried out a search for host galaxies for RRATs that are at the edge of our Galaxy and therefore present a possibility of being an FRB. We did not find any host galaxy which is within the search beam radius of each of these RRATs. This study suggested that a search for host galaxies should be carried out for future RRAT discoveries for which the dispersion measure (DM) is within the uncertainty of the Galactic free electron density model since these RRATs could be residing in nearby galaxies and could therefore be FRBs.

We also investigated the FRB population by carrying out Monte Carlo simulations of FRBs which have different energy and luminosity distributions. From the maximum likelihood analysis, we found that the known population of FRBs can be best expressed with having Gaussian distributed energy values and has a luminosity distribution that is a power law. The mean energy and luminosity of this distribution were determined to be $4.7 \times 10^{41} \mathrm{ergs}$ and $1.1 \times 10^{45} \mathrm{ergs} \mathrm{s}^{-1}$ respectively. We concluded that the need to have a power law luminosity distribution implies that FRBs are unlikely to be standard candles. Our main assumption in this analysis was that FRBs are uniformly distributed in the sky (model 1). For the best-fit model, we simulated a distribution that followed star formation history (model 2). We performed Kolmogorov-Smirnov test comparing these two models with each other and with the known sample. From these tests, we conclude that the DM distributions indicate that FRB progenitors do follow the cosmic star formation history. We demonstrate that the two models are distinguishable if the known sample contains at least 100 FRBs. 


\section{Acknowledgements}

This dissertation would not have been possible without the support and encouragement of many people. Firstly, I would like to thank my advisor, Dr. Duncan Lorimer, for all his guidance from the time I joined Department of Physics and Astronomy at West Virginia University and for all the time and effort he put into helping me with my research which made Grad school an enriching experience for me. I consider myself very lucky to have him as my advisor. Over the years he kept me motivated with his encouragement and positive feedback, which enabled the successful completion of this work. I got to learn a lot of concepts in radio astronomy, pulsars from him through classes and meetings. He patiently helped in developing my programming skills in which I had little experience, data analysis skills, presentation skills, and good scientific writing skills which I am still trying to achieve. All the valuable discussions and the training he provided will benefit me throughout my career.

I would like to thank my dissertation committee members, Dr. Maura McLaughlin, Dr. D. J. Pisano, Dr. Paul Cassak, and Dr. Kevin Bandura for their useful feedback and comments on this dissertation. Special thanks to Maura with whom I worked in a couple of projects. During that time and even otherwise, she has been a great mentor too and I have learned a lot from her about pulsars and astronomy in general. I would also like to thank other faculty members who taught me physics classes and specially the Astro faculty for maintaining a friendly atmosphere in the department which I found extremely encouraging for the last 6 years.

I would like to thank the department Chair Dr. Earl Scime and Graduate student advisor Dr. Mark Koepke for being very helpful and all the department office staff, especially Sherry Puskar and Viola Bryant for their efficient handling of all the necessary paperwork. A big thank you to Nate for fixing all the computer issues, helping me with parallel processing, and always willing to answer all my silly questions. I would also like to thank Dr. Sam Bates for helping me with shell scripting to optimize the pipeline for data processing in the first year.

I am thankful to Prof. Avi Loeb for giving me an opportunity to work at the Harvard-Smithsonian Center for Astrophysics which resulted in one of the chapters of this dissertation. All of the daily interesting discussions about pulsars and fast radio bursts were really insightful. I would also like to thank my other collaborators, Dr. Richard Prestage, Dr. Jeffrey Newman, and Dr. Bing Zhang for useful teleconferences. I am truly grateful to Dr. Chayanika Shah and Dr. Shashikumar Chitre Sir for pursuing me to go to Grad school.

I am very much grateful to my fellow graduate students Nipuni Palliyaguru, Lucas Hunt, Pete Gentile, Joe Swiggum, Fernando Cardoso, Will Armentrout, Gary Lander, Amy Sardone, Kaustubh Rajwade, Golnoosh Golpayegani, Belinda Cheeseboro, Ashok Choudhary, and Nihan Pol for great company, useful conversations on research projects and tolerating me in tough times. You all made coming to office 
everyday more exciting and I will miss the long, non-productive but entertaining lunch conversations.

A big thank you to my dear friends at WVU, Rutika Bhapkar, Siddhita Shirsat, Saurabh Chaudhari, Pratik Pednekar, Sanket Joshi, and Kiran Chaudhari who were like family away from home. Special thanks to my closest friends Bipasha Chakraborty, Anshulee Palkar, Asmita Redij, Milind Kawli, and Anuja Parker who managed to be there for me when I needed to talk to them in spite of being on different continents. I would also like to thank my family in the US, my uncle Nandkishor Muley and cousin Anagha Bock who made sure I was doing fine every now and then.

I owe my sincere thanks to my parents, Ashok Rane and Asmita Rane for everything they have done to bolster my interest in Astronomy since childhood and for all the love and support throughout this long journey in Grad school. I would like to thank my brother-in-law Amaury Groc for the support and for having the enthusiasm in what I do, my sister Anuya Rane and my cutest niece and nephew Paloma and Marius for making my weekends relaxing through skype calls. And last but not the least, many thanks to my dearest husband Mukut Ranjan Kalita who went on a ride along with me with many ups and downs while being in Grad school himself. He kept me pushing to overcome day-to-day difficulties in Grad school, helped me in understanding physics concepts when I needed and provided all the moral support until the finish line. 


\section{Table of Contents}

List of Tables $\quad$ viii

List of Figures $\quad$ ix

List of Abbreviations and Symbols $\quad$ xi

1 Introduction 1

1.1 Radio transient sky . . . . . . . . . . . . . . . . . . . 1

1.2 Pulsars . . . . . . . . . . . . . . . . . . . 3

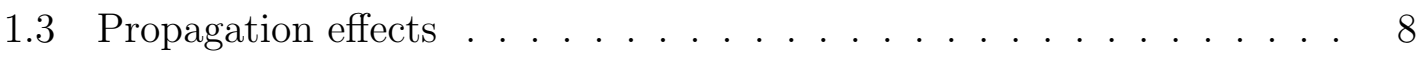

1.3.1 Dispersion . . . . . . . . . . . . . . . . 8

1.3 .2 Scattering . . . . . . . . . . . . . . . 11

1.4 Searching for pulsars . . . . . . . . . . . . . . . . . . . . . . . . . . . . . . . . . 13

1.4.1 De-dispersion . . . . . . . . . . . . . . . 13

1.4 .2 Periodicity search . . . . . . . . . . . . . . . . 14

1.5 Rotating Radio Transients . . . . . . . . . . . . . . . . . 15

1.6 Fast Radio Bursts . . . . . . . . . . . . . . . . . . . . . . . . . . . . . . . . . . . . 17

1.6.1 History . . . . . . . . . . . . . . . . . 17

1.6.2 Repeating FRB . . . . . . . . . . . . . . . . 24

1.6.3 Dispersion measure . . . . . . . . . . . . . . . . 26

1.6.4 Scattering . . . . . . . . . . . . . . 27

1.6.5 Polarization . . . . . . . . . . . . . . . 31

1.6.6 Progenitor models . . . . . . . . . . . . . . . . 34

1.6.7 FRBs as cosmological tools . . . . . . . . . . . . . . 40

1.7 Dissertation Outline . . . . . . . . . . . . . . . 47

2 A search for rotating radio transients and fast radio bursts in the Parkes high-latitude pulsar survey 48

2.1 Introduction . . . . . . . . . . . . . . . . . . . . . 48

2.2 Survey parameters . . . . . . . . . . . . . . . . . . . . . . . . . . . . . . . . . . 49

2.3 Single-pulse search method . . . . . . . . . . . . . 50

2.3.1 Dedispersion . . . . . . . . . . . . . 50

2.3.2 Single-pulse search .................. 51

2.3.3 Detection criteria .................. 53

2.3.4 Manual inspection ... . . . . . . . . . . . . 54

2.4 Results . . . . . . . . . . . . . . . . . . . 55

2.5 Energy measurements . . . . . . . . . . . . . . . 56

2.6 Intermittency measure . . . . . . . . . . . . . . . . . . . . 58

2.7 Conclusions .......................... 61 
3 Event rate of Fast Radio Bursts 62

3.1 Introduction . . . . . . . . . . . . . . . . . . . . 62

3.2 Bayesian Approach . . . . . . . . . . . . . . . . . 63

3.3 Discussion . . . . . . . . . . . . . . . . . . . 64

3.4 All-sky event rate of FRBs . . . . . . . . . . . . . . . . . 66

3.5 Additional Parkes surveys and modified event rate . . . . . . . . . . 71

3.6 Conclusions . . . . . . . . . . . . . . . . . 73

4 Searching for host galaxies of potentially extragalactic RRATs 76

4.1 Introduction . . . . . . . . . . . . . . . . . . . 76

4.2 Galactic free electron density models . . . . . . . . . . . . . . 79

4.3 RRAT sample ...................... . . . 83

4.4 Results ....................... 83

4.4.1 RRAT J1332-03 . . . . . . . . . . . . . . . . 85

4.4.2 RRAT J0156+04 . . . . . . . . . . . . . . . 86

4.4.3 RRAT J1603+18 .................. . . 87

4.4.4 RRAT J1354+24 . . . . . . . . . . . . . . . . . . . 87

4.4.5 RRAT J0837-24 .................. . . . . 87

4.4.6 RRAT J1433+00 . . . . . . . . . . . . . . . . . 88

4.5 Discussion . . . . . . . . . . . . . . . . . . . . . 90

4.6 Summary and conclusions . . . . . . . . . . . . . . . . 90

5 Empirical Population Models for Fast Radio Bursts 92

5.1 Introduction . . . . . . . . . . . . . . . . . . . . . . 92

5.2 Comparison sample . . . . . . . . . . . . . . . . . 96

5.3 Energy and luminosity distributions . . . . . . . . . . . . . . . . 98

5.4 Redshift distribution . . . . . . . . . . . . . . . . . . . . . 99

5.5 DM contributions . . . . . . . . . . . . . . . . 101

5.5.1 Galactic contribution ................... 101

5.5.2 IGM contribution . . . . . . . . . . . . . . . . 102

5.5.3 Host Galaxy contribution . . . . . . . . . . . . . 103

5.6 Contributions to FRB widths . . . . . . . . . . . . . . . 104

5.6.1 Dispersive delay . . . . . . . . . . . . . . . . 105

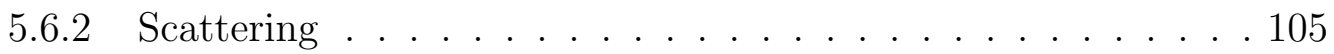

5.7 Peak fluxes of FRBs . . . . . . . . . . . . . . . . . . . . . . . . . . . . . . . . . . . . . . . .

5.8 Signal-to-noise ratio . . . . . . . . . . . . . . . . . . . . 108

5.9 Simulation procedure . . . . . . . . . . . . . . . . . 110

5.10 Maximum likelihood analysis . . . . . . . . . . . . . . . . . . . . . . . . . . . . . . . . . . . . . . .

5.11 Results . . . . . . . . . . . . . . . . . . . 115

5.12 Discussion . . . . . . . . . . . . . . . . . . . . 117

5.12.1 The $\log N-\log S$ relation of FRB events . . . . . . . . . . . . 121

5.12.2 Tracking star formation history . . . . . . . . . . . . . 123

5.13 Conclusions . . . . . . . . . . . . . . . . . . . 130

6 Conclusions and Future Directions 132 
A Pulse broadening due to DM step size 136

B Contribution to DM from the intergalactic medium 138

$\begin{array}{lll}\text { C } & \text { MCMC results for all models } & 142\end{array}$ 


\section{List of Tables}

1.1 List of FRB follow-up observations . . . . . . . . . . . . . . . 25

2.1 Properties of pulsars detected in the PH survey . . . . . . . . . 57

3.1 Various Parkes survey parameters and our results . . . . . . . . . 70

4.1 Properties of the subset of RRATs included in our sample . . . . . . 86

4.2 The extragalactic objects seen within the beam search radius . . . . . 89

5.1 List of observables for the comparison sample . . . . . . . . . . . 97

5.2 System parameters for the Parkes HTRU high latitude survey . . . . 109

5.3 The initial set of parameters for each model . . . . . . . . . . . . . 114

5.4 The optimal parameters for each model . . . . . . . . . . . . . 117

5.5 Comparing star formation history and constant density model . . . . 127

5.6 Results of the Kolmogorov-Smirnov test . . . . . . . . . . . . . . 128 


\section{List of Figures}

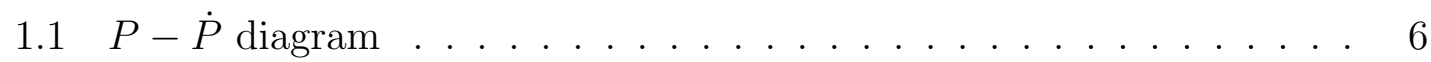

1.2 Dedispersion . . . . . . . . . . . . . . . . 9

1.3 Simple scattering model . . . . . . . . . . . . . . . . . . . 11

1.4 Time series . . . . . . . . . . . . . . . . . . . . 15

1.5 Lorimer burst detection . . . . . . . . . . . . . . . . . . 18

1.6 The DM-latitude dependence for Galactic pulsars and RRATs . . . . 20

1.7 FRB and peryton distributions as a function of local time and DM . 24

1.8 The DM latitude dependence for all radio transients . . . . . . . . . . 28

1.9 The scattering timescales for radio transients . . . . . . . . . . . . . 29

1.10 FRB profiles showing scattering . . . . . . . . . . . 30

1.11 Current observational measurements of the low redshift baryon census 41

1.12 Dispersion in DM for FRBs with $z \sim 1 \ldots \ldots . \ldots . \ldots 42$

1.13 Estimating cosmological parameters from observations . . . . . . 44

1.14 Estimating the dark energy density parameter . . . . . . . . . 46

2.1 Example output of single-pulse search . . . . . . . . . . . . . 54

2.2 False FRB detection . . . . . . . . . . . . . . . . 55

2.3 Normalized energy histograms . . . . . . . . . . . . . . . . . 59

2.4 Intermittency measures of pulsars $\ldots \ldots \ldots \ldots$

3.1 The modified FRB event rates . . . . . . . . . . . . . . 68

3.2 The combined posterior PDF of the FRB event rate . . . . . . . . . 69

3.3 The modified posterior PDF . . . . . . . . . . . . . 74

4.1 DM plot for all radio transients using NE2001 model . . . . . . . . . 81

4.2 DM plot for all radio transients using YMW16 model . . . . . . . . . 82

4.3 Histograms for RRATs and FRBs . . . . . . . . . . . . . . 83

4.4 A zoomed in DM plot for RRATs . . . . . . . . . . . . . 84

4.5 DM plot for RRATs in our sample . . . . . . . . . . . 85

5.1 Scatter plots showing the parameter space for the observed FRB sample 98

5.2 Co-moving distance as a function of redshift . . . . . . . . . 100

5.3 Flowchart summarizing the simulation procedure . . . . . . . . . 111

5.4 MCMC results for the best-fit model . . . . . . . . . . . . . . 116

5.5 Energy distribution of the detectable modeled FRB population . . . . 118

5.6 Luminosity distribution of the detectable modeled FRB population . 119

5.7 Comparing modeled FRBs with known population . . . . . . . . . . 120

5.8 Peak fluxes versus $\mathrm{S} / \mathrm{N}$ of undetectable FRBs . . . . . . . . . . . 120

5.9 Comparing 20 modeled FRBs with known population . . . . . . . . 121

5.10 Sky distribution of modeled and known FRBs. . . . . . . . . . . . . 122

5.11 Fraction of detectable FRBs in a co-moving shell . . . . . . . . . . . 122

5.12 The $\log N-\log S$ relation for sources in the Euclidean universe . . . . 124

5.13 The $\log N-\log S$ curves for known and modeled FRBs . . . . . . . . . 124 
5.14 The history of cosmic star formation . . . . . . . . . . . . . 125

5.15 The redshift distribution of FRBs tracking cosmic SFH. . . . . . . . . 126

5.16 The redshift distributions of FRBs for two models . . . . . . . . . . . 127

5.17 Results of the Kolmogorov-Smirnov test . . . . . . . . . . . . . . . . 129

C.1 MCMC results for model A1 . . . . . . . . . . . . . . . . 143

C.2 MCMC results for model A2 . . . . . . . . . . . . . . . . . . . 143

C.3 MCMC results for model B1 . . . . . . . . . . . . . . . . . . 144

C.4 MCMC results for model B2 . . . . . . . . . . . . . . . . . . . 144

C.5 MCMC results for model C1 . . . . . . . . . . . . . . . . . 145

C.6 MCMC results for model C2 . . . . . . . . . . . . . . . . . 145 


\section{List of Abbreviations and Symbols}

\begin{tabular}{|c|c|}
\hline $\mathrm{AGN}$ & Active Galactic Nuclei \\
\hline ATCA & Australia Telescope Compact Array \\
\hline ATNF & Australia Telescope National Facility \\
\hline$d$ & Distance to the pulsar or FRB \\
\hline $\mathrm{DM}$ & Dispersion measure \\
\hline FFT & Fast Fourier Transform \\
\hline FRB & Fast Radio Burst \\
\hline GP & Giant Pulse \\
\hline GRB & Gamma Ray Burst \\
\hline GBT & Green Bank Telescope \\
\hline$l$ & Galactic longitude \\
\hline$b$ & Galactic latitude \\
\hline IGM & Intergalactic Medium \\
\hline ISM & Interstellar Medium \\
\hline HII & Ionized Hydrogen \\
\hline HTRU & High Time Resolution Universe \\
\hline$\Lambda-\mathrm{CDM}$ & $\Lambda$-Cold Dark Matter \\
\hline LMC & Large Magellanic Cloud \\
\hline $\mathrm{MCMC}$ & Markov chain Monte Carlo \\
\hline MSP & Millisecond pulsar \\
\hline MW & Milky Way \\
\hline NS & Neutron star \\
\hline$I$ & Neutron star moment of inertia $\left(=10^{45} \mathrm{~g} \mathrm{~cm}^{2}\right)$ \\
\hline PSR & Pulsar \\
\hline$\omega$ & Rotational angular frequency \\
\hline RRAT & Rotating radio transient \\
\hline $\mathrm{SM}$ & Scattering Measure \\
\hline $\mathrm{S} / \mathrm{N}$ & Signal-to-noise ratio \\
\hline$M_{\odot}$ & Solar mass \\
\hline SGR & Soft Gamma Repeater \\
\hline $\mathrm{SMC}$ & Small Magellanic Cloud \\
\hline$c$ & Speed of light \\
\hline$P$ & Spin period \\
\hline$\dot{P}$ & Spin period derivative \\
\hline$\dot{E}$ & Spin-down luminosity \\
\hline $\mathrm{SFH}$ & Star formation history \\
\hline $\mathrm{SNe}$ & Supernovae \\
\hline SNR & Supernova remnant \\
\hline SLSNe & Super Luminous Supernovae \\
\hline$B$ & Surface magnetic field strength \\
\hline VLA & Very Large Array \\
\hline WD & White Dwarf \\
\hline WIMP & Weakly Interacting Massive Particles \\
\hline
\end{tabular}




\section{Chapter 1}

\section{Introduction}

\subsection{Radio transient sky}

A large number of transient sources are currently known. The known classes of sources emit on timescales ranging from nanoseconds up to as long as years but still short relative to the typical timescales of events in the Universe. Sources from the planetary scale to super-massive black holes can exhibit transient behavior. Astronomers have been observing transient phenomena in the sky for centuries. Some of the early examples include recording of the nearby supernova (SN) that created the Crab Nebula in AD 1054, and SNe of AD 1604, 1572, 1181, 1006. There are some records of possible novae or SNe before AD 1000 as well. All of these historical observations are described in detail in Stephenson \& Green (2002). Since then, telescopes probing the entire electromagnetic spectrum have detected many other classes of transient sources. These include the Sun, planets, brown dwarfs, flare stars, X-ray binaries, ultra-high energy particles from cosmic rays, $\gamma$ ray bursts, maser flares, active galactic nuclei, radio supernovae, and pulsars (Cordes et al., 2004). Observations of the radio sky in the last century have resulted in the discovery of several classes of radio transients. These transient sources offer unique opportunities to study the astrophysics of these objects and the variability timescales could be used as an early diagnostic of source class in future radio transient surveys. 
The Sun is the brightest radio source in the sky, and varies over an 11-year solar cycle. The solar emission at 10-40 MHz correlating with periods of high sunspot activity was the first radio transient phenomenon observed (Appleton, 1945). The radio bursts from the Sun are labelled as Types I-V and range from a few seconds up to several weeks and are observed at frequencies below $300 \mathrm{MHz}$ (Dulk, 1985). Most of these bursts are caused by variations in the solar plasma. When electrons are accelerated to energies of a few $\mathrm{keV}$ in the solar active regions, they travel outward through the corona and solar winds. Since the electron density decreases as a function of height in the solar atmosphere, the plasma frequency (which is proportional to the square of the electron density) also decreases with height. At each successive height, electrons excite plasma oscillations at the local plasma frequency generating radio emission. The planets in our solar system which have magnetospheres including Earth, Jupiter, Saturn, Uranus, and Neptune show auroral radio emission that lasts a few seconds (Zarka, 1998). This emission originating in the high magnetic latitude regions is caused by energetic electrons striking the atmosphere. Several authors (Farrell et al., 1999; Zarka et al., 2001; Lazio et al., 2004) have suggested that extrasolar planets would also produce similar bursts and detection of radio emission can lead to direct detection of extrasolar planets.

Outside of our Solar System, the nearby Galactic flare stars (red dwarfs) emit solar-like bursts ranging from milliseconds to days. These flares are broadband emission. Ultra-cool brown dwarfs and low mass stars have also been seen to emit periodic radio emission (Hallinan et al., 2007) of the order of $100 \mu \mathrm{Jy}$ at frequencies between 5 and $8 \mathrm{GHz}$. This is the stimulated spectral line emission, typically in 
the microwave portion of the electromagnetic spectrum known as the maser (Microwave Amplification by Stimulated Emission of Radiation) emission and confirm the existence of stellar magnetic fields of the order of a few $\mathrm{kG}$. These bursts are rotationally driven from a star with a dipole field, similar to pulsars. The pulses last for several minutes and repeat on timescales of 2-3 hours (Hallinan et al., 2008).

Earth is constantly bombarded with ultra-high energy cosmic rays with energies between $10^{18}-10^{20} \mathrm{eV}$. This bombardment results in nano-second duration radio bursts at frequencies of a few $\mathrm{Hz}$ to a few hundred $\mathrm{MHz}$ that are extremely bright $\left(\sim 10^{6}\right.$ Jy; Huege \& Falcke 2003). But since the cosmic magnetic fields deflect their paths, their source of origin remains unidentified.

Radio emission has also been detected from gamma-ray-bursts (GRBs) and SNe in the form of a radio afterglow. The collision of the supernova shock wave into the ionized circumstellar medium produces incoherent synchrotron radiation which is visible across a range of radio frequencies (Weiler, 1983).

\subsection{Pulsars}

As a massive star runs out of fuel in its core, it collapses in on itself, creating a massive explosion called a supernova. A neutron star is what is left of the core after this explosive death. The existence of a neutron star as the end point of stellar evolution was proposed by Baade and Zwicky in 1934. Over thirty years later, Jocelyn Bell at Cambridge found sources which emitted individual pulses with durations of 10s of milliseconds (Hewish et al., 1968), suggesting associations 
with oscillations of white dwarfs or neutron stars from within the Galaxy. This source is designated as PSR B1919+21; indicating its position in right ascension and declination with B corresponding to the epoch Besselian 1950. Since then the field of pulsar astronomy has evolved by carrying out numerous surveys with large radio telescopes. At the time of writing, the ATNF (Australia Telescope National Facility) data network has recorded about 2613 pulsars, including sources in nearby satellite galaxies (Manchester et al., 2005).

Pulsars are excellent tools to study a wide variety of astrophysical phenomena, including neutron star physics, the interstellar medium, general relativity, and gravitational waves. The incredibly stable periods of pulsars, especially the millisecond pulsars (MSPs), make pulsars excellent probes of extreme physics. For example, studies of the interior of a neutron star provides insight into understanding of the properties of matter under extreme pressure. If the pulsar is in a binary system, then the periodic Doppler shifts in the pulse arrival times can be analyzed to reveal the presence of the companion, its mass, and how the companion star is evolving. Pulsars can also be used to study the Galactic magnetic field. Pulsar magnetospheres provide tools to study plasma physics under extreme conditions. For detailed discussion on these methods, see Lorimer \& McLaughlin (2010); Kramer (2016).

According to the commonly accepted model, the pulsar emission is from a rapidly rotating, highly magnetized neutron star and is generated in the open magnetic field line region at the star's magnetic poles. As the star spins, the highly focused beams of radiation from the poles sweep across the sky like a lighthouse and 
if one of these beams intersects the line of sight with Earth the star is seen as a pulsar (Gold, 1968; Pacini, 1968). When a neutron star is formed, angular momentum is conserved, hence it starts spinning rapidly and due to the conservation of magnetic flux, the magnetic field also increases. The surface magnetic fields of pulsars range from $10^{8}-10^{14}$ Gauss. A typical radius is $10 \mathrm{~km}$ and the observed masses are in the range of 1.4 to $2 M_{\odot}$ (Özel \& Freire, 2016). The emission that we see is made possible by the steady conversion of rotational kinetic energy into acceleration of particles along open magnetic field lines at the polar caps. As a result of magnetic dipole braking radiation, pulsars gradually slow down.

Most pulsars have spin periods $(P)$ of the order of a few hundred milliseconds and slow down rate $(\dot{P})$ near $10^{-15} \mathrm{~s} \mathrm{~s}^{-1}$ as can be seen in Figure 1.1, known as the period-period derivative plot or ' $P-\dot{P}$ diagram' for pulsars. There is another population of pulsars that spin with millisecond periods and $\dot{P}<10^{-19} \mathrm{~s} \mathrm{~s}^{-1}$ (Özel \& Freire, 2016). These are known as millisecond pulsars (MSPs) and are seen in the lower left of the $P-\dot{P}$ diagram. The very first MSP $($ B1937+21) with a period of $\sim 1.6 \mathrm{~ms}$ was discovered in 1982 using the Arecibo telescope (Backer et al., 1982). MSPs are believed to be old neutron stars that have been spun-up by the accretion of matter and angular momentum from a binary companion to the pulsar and are often referred to as "recycled" pulsars. The fastest spinning pulsar that has been observed is PSR J1748-2446ad, with a spin period of 1.39 ms (Hessels et al., 2006). Lorimer (2008) reviews the properties and evolution of millisecond pulsars.

Pulsar emission is relatively broadband i.e. most pulsars are observable from GHz to a few tens of MHz. Gamma-ray emission has also been detected from both 


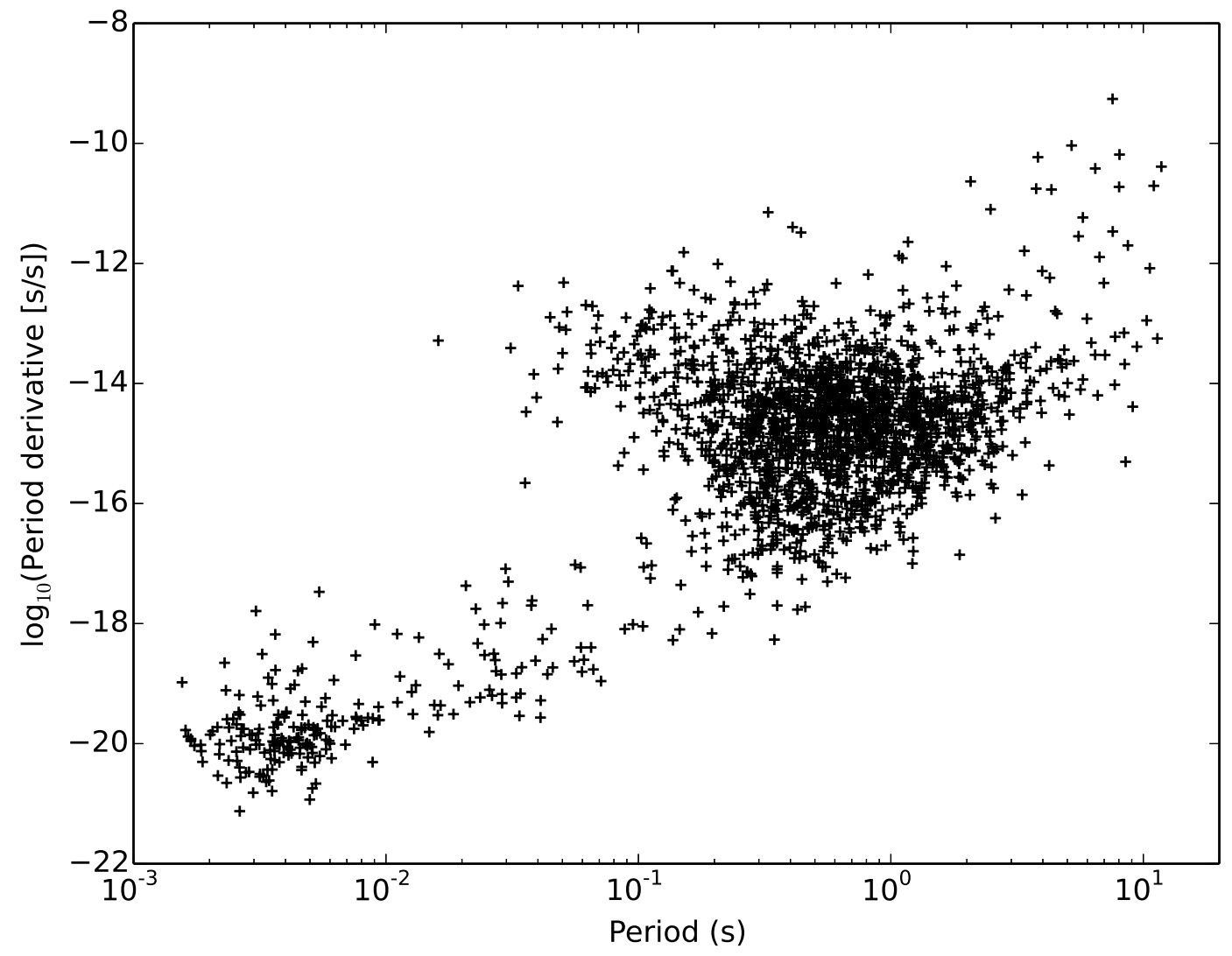

Figure 1.1: $P-\dot{P}$ diagram for Galactic pulsar population. 
young and old pulsars (Abdo et al., 2009). The rapid rotation of the neutron star, combined with its strong magnetic field, leads to a large electric field which accelerates particles to extremely high energies. These 'primary' particles emit gamma rays which decay into 'secondary' electron-positron pairs, and the radio emission is attributed to these outflowing secondary pairs. The gamma-ray emission peak is typically offset from the peak radio pulse suggesting that emission at high energies takes place higher in the pulsar magnetosphere, further from the surface than the narrowly-beamed radio emission (Weltevrede et al., 2010).

Another type of neutron star, known as a magnetar, has an extremely strong magnetic field $\left(B \sim 10^{15}\right.$ Gauss). These are seen in the top right corner of the $P-\dot{P}$ diagram. They have very long spin periods with $P>2 \mathrm{~s}$ and $\dot{P}>10^{-13} \mathrm{~s} \mathrm{~s}^{-1}$. In magnetars, the magnetic field decay powers the emission of high-energy electromagnetic radiation, particularly X-rays and gamma rays. The existence of such objects was proposed in 1992 by Duncan \& Thompson (1992) but the first magnetar detection as a burst of gamma rays was reported in 1979 (Mazets, Golenetskij, \& Guryan , 1979; Mazets et al., 1979; Mazets \& Golenetskii, 1981). Despite their large magnetic fields, their spin-down energies are far too low to account for their X-ray luminosities. These are known as anomalous X-ray pulsar (AXP). Also, soft gamma ray repeaters (SGRs) are believed to be magnetars. Currently, 29 magnetars are known. More details on magnetar populations and their emission mechanism can be found in a recent review by Kaspi \& Beloborodov (2017). 


\subsection{Propagation effects}

A radio telescope records pulses across some observing bandwidth with a particular sampling rate. The ionized material in the interstellar medium (ISM) affects this pulsar signal as it travels from the source through the Galaxy before reaching the telescope. These effects are dispersion, scintillation and scattering.

\subsubsection{Dispersion}

Pulse dispersion refers to the frequency dependence of the group velocity of radio waves, seen as the sweep in the frequency versus time plot (Figure 1.2). It causes the higher frequency pulses to arrive earlier at the telescope than their lower frequency counterparts. To derive the time delay due to this dispersion, we define the refractive index $\mu$ of the ionized gas for a wave with observing frequency $\nu$

$$
\mu=\sqrt{1-\frac{\nu_{\mathrm{p}}^{2}}{\nu^{2}}},
$$

where the plasma frequency $\nu_{\mathrm{p}}$ is

$$
\nu_{\mathrm{p}}=\sqrt{\frac{n_{\mathrm{e}} e^{2}}{\pi m_{\mathrm{e}}}} .
$$

Here $e$ and $m_{\mathrm{e}}$ are the charge and mass of an electron respectively, and $n_{\mathrm{e}}$ is the electron number density. A typical interstellar electron density is $0.03 \mathrm{~cm}^{-3}$ (GomezGonzalez \& Guelin, 1974), corresponding to a plasma frequency of $1.5 \mathrm{kHz}$. The group velocity at which the pulse travels is $v_{\mathrm{g}}=c \mu$ and it should be less than $c$ for 


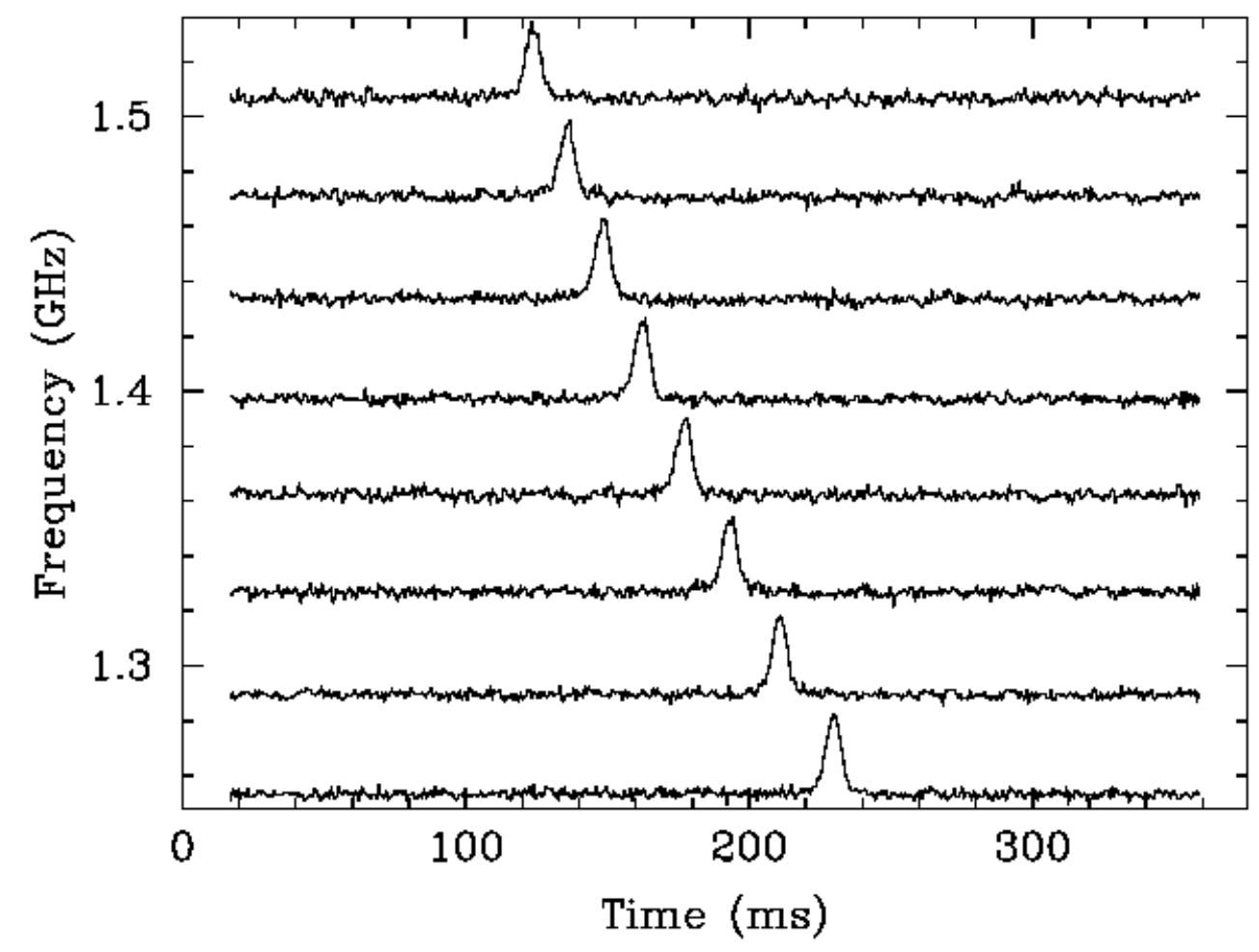

Figure 1.2: The dispersion in the frequency versus time plot for PSR J0837-4135 showing that the higher frequency components arrive earlier than the lower frequency components. 
the wave to propagate. Over a distance $d$, the pulse will be delayed in time with respect to the free-space travel time as

$$
t=\int_{0}^{d} \frac{d l}{v_{\mathrm{g}}}-\frac{d}{c}
$$

Substituting for $v_{\mathrm{g}}$

$$
t=\frac{e^{2}}{2 \pi m_{\mathrm{e}} c} \frac{\int_{0}^{d} n_{\mathrm{e}} d l}{\nu^{2}}-\frac{d}{c} .
$$

The integral of column density of free electrons along the line of sight is the dispersion measure (DM), i.e.,

$$
\mathrm{DM}=\int_{0}^{d} n_{\mathrm{e}} d l .
$$

Across the observing band, the delay between consecutive frequency channels

$$
\Delta t \cong 4.15 \times 10^{6} \times \mathrm{DM}\left(\frac{1}{\nu_{1}^{2}}-\frac{1}{\nu_{2}^{2}}\right) \mathrm{ms} .
$$

$\mathrm{DM}$ is given in units of $\mathrm{cm}^{-3} \mathrm{pc}$ and for most practical cases in which the central observation frequency $\nu$ is much greater than the channel width $\Delta \nu$, the dispersive delay across a frequency channel of width $\Delta \nu$ is

$$
\Delta t \cong 8.3 \times 10^{6} \times \mathrm{DM} \times \frac{\Delta \nu}{\nu^{3}} \mathrm{~ms} .
$$

In this expression, $\Delta \nu$ and $\nu$ are in MHz. The free electron density, $n_{\mathrm{e}}$, varies along different lines of sight in the Milky Way. The NE2001 model (Cordes \& Lazio, 2002) 


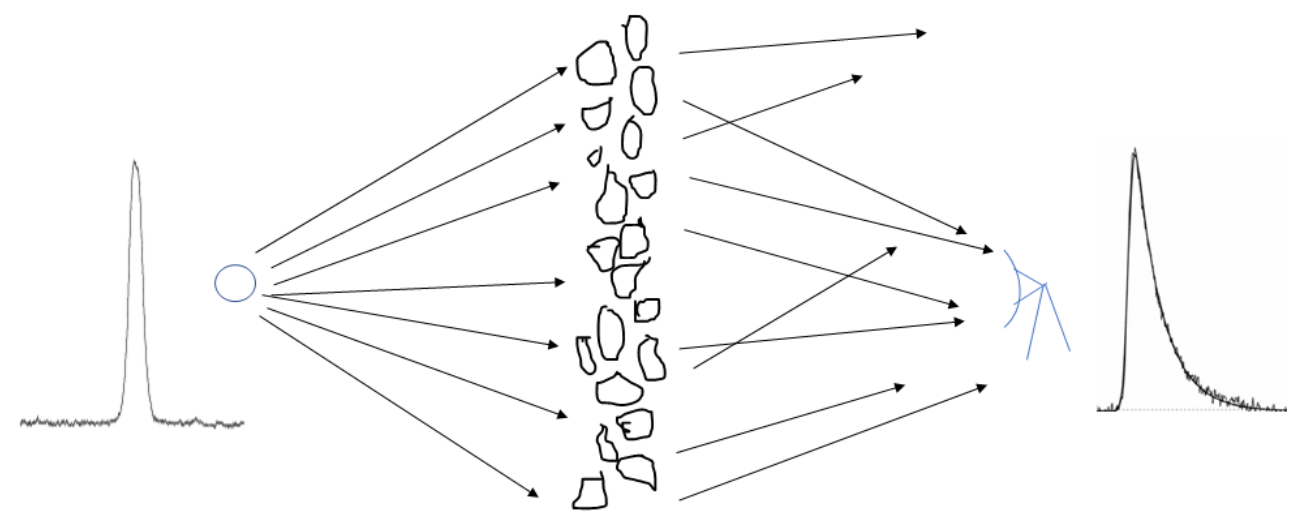

Figure 1.3: Multipath propagation for the pulsar radiation that travels through inhomogeneities in the turbulent plasma. The effect is seen as exponential tails in the pulse profile.

which describes the structure of ionized gas in the Galaxy gives the estimated $n_{\mathrm{e}}$ along every line of sight and is the most widely used model. Therefore, if a pulsar is observed across a frequency band, the DM can be inferred using Equation 1.7. Assuming the NE2001 model, the DM can be used to estimate the distance to the pulsar by numerically integrating Equation 1.5.

\subsubsection{Scattering}

The ionized medium is also turbulent and as a result, the waves are scattered and take slightly longer paths to reach the observer relative to radiation that arrives along the direct line of sight. This smearing arising due to multipath propagation as the radiation travels through inhomogenities in the turbulent plasma is seen as exponential tails in the pulse profiles (see Figure 1.3) and can give information about the intervening medium. 
The ionized interstellar medium also has some small-scale irregularities. This interstellar scintillation causing fading of radio signals from pulsars was first identified by Rickett (1969) who showed that these fluctuations could be clearly distinguished from other kinds of fluctuation intrinsic to pulsars. This turbulence has both diffractive and refractive effects on the pulsar radiation. Diffractive scintillation can be observed as short timescale ( minutes) fluctuations in the intensity of the pulsar signal in narrow $(\sim 1 \mathrm{MHz})$ frequency bands (e.g., Cordes \& Rickett, 1998). Scheuer (1968) developed a basic model to explain this scintillation. The model assumes that the interstellar medium is a thin screen of inhomogeneous matter, placed midway between the pulsar and the observer. The phase perturbations of the signal produced by such a screen are correlated over a scintillation bandwidth $\Delta \nu_{\text {scatt }} \propto \nu^{4}$. Refractive scintillation on the other hand causes longer term, less deep variations in the flux density (Sieber, 1982). The scattering timescale, $t_{\text {scatt }}$, is inversely proportional to $\Delta \nu_{\text {scatt }}$. Measurements show that more distant pulsars with higher DM, in general, will be more scattered (Bhat et al., 2004). The scattering measure (SM) gives the amount of turbulence along the line of sight

$$
\mathrm{SM}=\int_{0}^{d} C_{\mathrm{N}}^{2} d l,
$$

where $C_{\mathrm{N}}$ is the local amplitude of the turbulence as a function of distance which is proportional to the square of the electron density. The scattering timescale is therefore proportional to the scattering measure. We can also compute the scattering measure for various models of the turbulence. 


\subsection{Searching for pulsars}

After the first discovery, numerous radio surveys were designed to discover pulsars and a few more bright pulsars were discovered through searches for individual pulses (Lyne \& Rickett, 1968; Staelin \& Reifenstein, 1968). However, soon after that, the primary method of searching for pulsars shifted to periodicity searches as it was more productive (Hulse \& Taylor, 1974) and it has still remained the most widely used method. In this standard strategy, initially the raw data are de-dispersed, which produces a time series that can be analyzed to search for pulses.

\subsubsection{De-dispersion}

The amount of dispersion seen in the Figure 1.2 is unique to each pulsar signal. When searching for a pulsar, the DM is initially unknown. Therefore, a search over many trial DMs is necessary to determine the correct DM. For each trial DM, the time delay is calculated using Equation 1.7 and the quadratic sweep across the observing band is removed by appropriately shifting the frequency channels. This step is known as de-dispersion. Then the signal is averaged over all channels to get a one dimensional time series for each trial DM (see Figure 1.4). For all astrophysical sources, DM $>0 \mathrm{pc} \mathrm{cm}^{-3}$. However, the raw data might have unwanted short duration or narrow channel radio frequency interference (RFI) which needs to be removed. RFI will typically have a DM of $0 \mathrm{pc} \mathrm{cm}^{-3}$ since $n_{\mathrm{e}}$ is negligible for terrestrial sources. Hence strong pulses at a DM of $0 \mathrm{pc} \mathrm{cm}^{-3}$ should be removed. The left panel of Figure 1.4 shows a time series for a DM value of $0 \mathrm{pc} \mathrm{cm}^{-3}$. It 
is very noisy. The time series in the right panel is obtained after correcting for dispersion at a DM of $147.3 \mathrm{pc} \mathrm{cm}^{-3}$ for PSR J0837-4135. It shows bright pulses above the noise level.

\subsubsection{Periodicity search}

The time series is analyzed to search for periodic signals. The periodic signal is usually hidden within the random noise. The search is performed in the frequency domain by taking the Fast Fourier transform (FFT) of the time series. The resultant power spectrum, which is a plot of the power of each Fourier component as a function of frequency, is searched for signals with a signal-to-noise ratio above some threshold, typically $S / N>5$. The spectra that have $2,4,8$, and 16 harmonics of the fundamental frequency are also searched. Individually these components may not be distinguishable from noise as the power from the pulsar is spread throughout these harmonics. Therefore they must be summed to maximize the detectability of the signal. With each summation, the noise decreases by a factor of $\sqrt{2}$ and the

powers of the components increase by $\sqrt{2}$. Every signal above this $\mathrm{S} / \mathrm{N}$ threshold in each of the DM trials is recorded as a candidate. Then the raw data is de-dispersed at this DM and folded at the period to make diagnostic plots that can be inspected manually to confirm the candidate as a pulsar. 

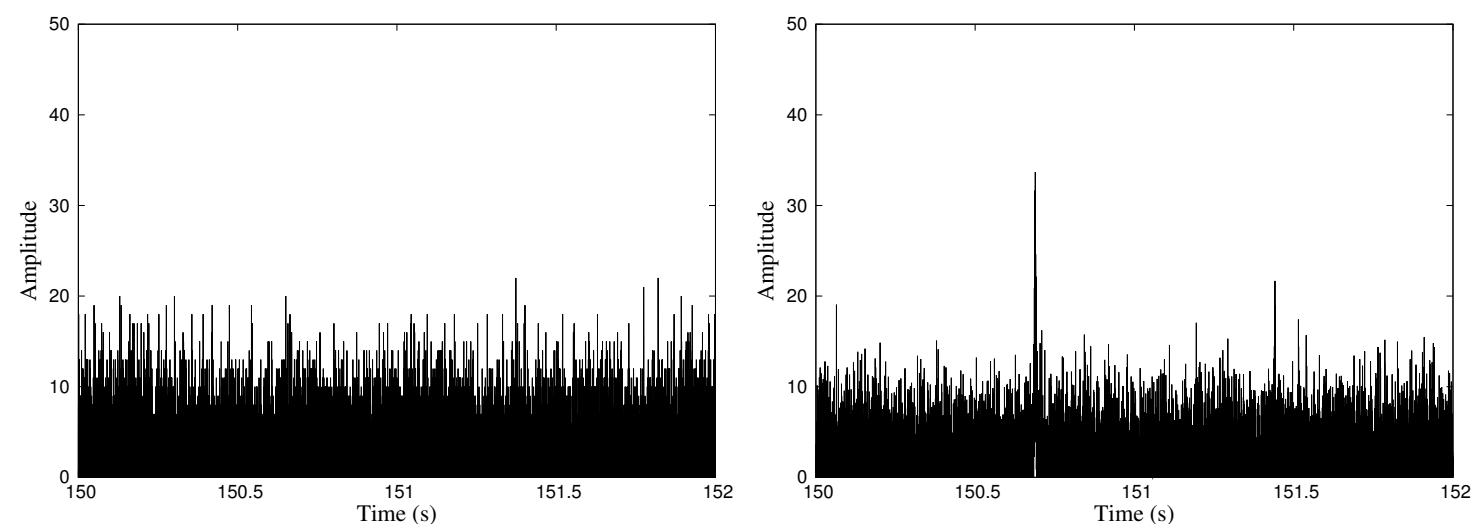

Figure 1.4: The left panel shows a time series for a DM value of $0 \mathrm{pc} \mathrm{cm}^{-3}$ which is very noisy and the time series in the right panel is obtained after correcting for dispersion at a DM of $147.3 \mathrm{pc} \mathrm{cm}^{-3}$ for PSR J0837-4135 which shows bright pulses above the noise level.

\subsection{Rotating Radio Transients}

Although the precise periodicity is the main characteristic of a pulsar signal, another class of transients, known as Rotating Radio Transients, or RRATs, are a group of Galactic pulsars that emit sporadic single bright pulses. These were first detected by McLaughlin et al. (2006) while processing the data from the Parkes Multibeam Pulsar Survey (PM survey; Manchester et al. 2001). There are 112 RRATs discovered so far ${ }^{1}$. The pulses from RRATs sometimes repeat but not regularly, like those of pulsars and some have been observed at only one epoch. Hence RRATs are easily detectable as single isolated pulses, rather than as a repeating signal. To detect RRATs, the single-pulse search is the only possible method. After the signal is de-dispersed as described above, a single-pulse search algorithm is applied instead of the periodicity search. This search method is explained in detail in Chapter 2.

\footnotetext{
${ }^{1}$ For an up-to-date list of RRATs, see http://astro.phys.wvu.edu/rratalog
} 
The lack of detection in a periodicity search suggests that RRATs are either much more weakly emitting, or in fact 'off', during times when we do not detect pulses. Despite this, by examining time differences between the arrival times of pulses, underlying periods have been estimated for almost 88 RRATs, for which enough pulses are seen together. Period derivatives have been estimated for 29 RRATs. The inferred source sizes, the underlying rotation periods, as well as the expected time-scales for transient behaviour all point towards RRATs being Galactic neutron stars.

In addition to RRATs, some pulsars are seen to emit giant single pulses occasionally. Their flux densities can exceed hundreds and thousands of times the mean flux density of regular pulses from the pulsar. For example, the giant pulses (GPs) from the Crab pulsar which led to its discovery show pulses with an intensity up to 1000 times that of an average pulse. The GPs are also very short in duration. Hankins et al. (2003) found pulses as short as $2 \mathrm{~ns}$ from the Crab pulsar. GPs from B1937+21 were observed for the first time in 1984 (Wolszczan et al., 1984) but were recognized later by Cognard et al. (1996). The duration of these GPs is short compared to the period of the pulsar, lasting on the order of 10 nanoseconds (Soglasnov et al., 2004). The amplitude distribution of GPs is a power-law (Popov \& Stappers, 2007), whereas the amplitude distribution of pulses from many pulsars is log-normal (Ritchings, 1976). 


\subsection{Fast Radio Bursts}

\subsubsection{History}

A renewed interest in finding other radio transients along with the discovery of RRATs led astronomers to perform single-pulse searches in addition to periodicity searches on pulsar survey data.

While processing the data from a survey of large and small Magellanic clouds (LMC, SMC) using the multibeam receiver from the 64-m Parkes radio telescope to search for pulsars and RRATs at 1.4 GHz, Lorimer et al. (2007) found an exceptionally bright burst that lasted a few milliseconds. The DM was found out to be anomalously high, compared to maximum DM that would be due to the electrons in the Milky Way in that direction. This event, known as the 'Lorimer burst', had a peak flux density of $\sim 33 \mathrm{Jy}$ and a DM of $375 \mathrm{pc} \mathrm{cm}{ }^{-3}$. The Galactic DM contribution along the line of sight of this burst predicted by the NE2001 model is 45 pc $\mathrm{cm}^{-3}$, i.e. only $12 \%$ of the total measured DM value. Therefore, the excess DM indicated that the source is located outside our Galaxy. Figure 1.5 shows an output of a single-pulse search analysis done by Lorimer et al. (2007) and their detection of the Lorimer burst. It is seen in the DM versus time plot as a strong pulse and has a $\mathrm{S} / \mathrm{N}>20$ as seen in the $\mathrm{S} / \mathrm{N}$ versus $\mathrm{DM}$ plot at the top right corner. This type of diagnostic plot obtained in the single-pulse search analysis is discussed in detail in $\S 2.3 .4$.

This burst remained debated for quite some time. A few years later, BurkeSpolaor et al. (2011) found bursts similar in nature at high DMs in Parkes data. 

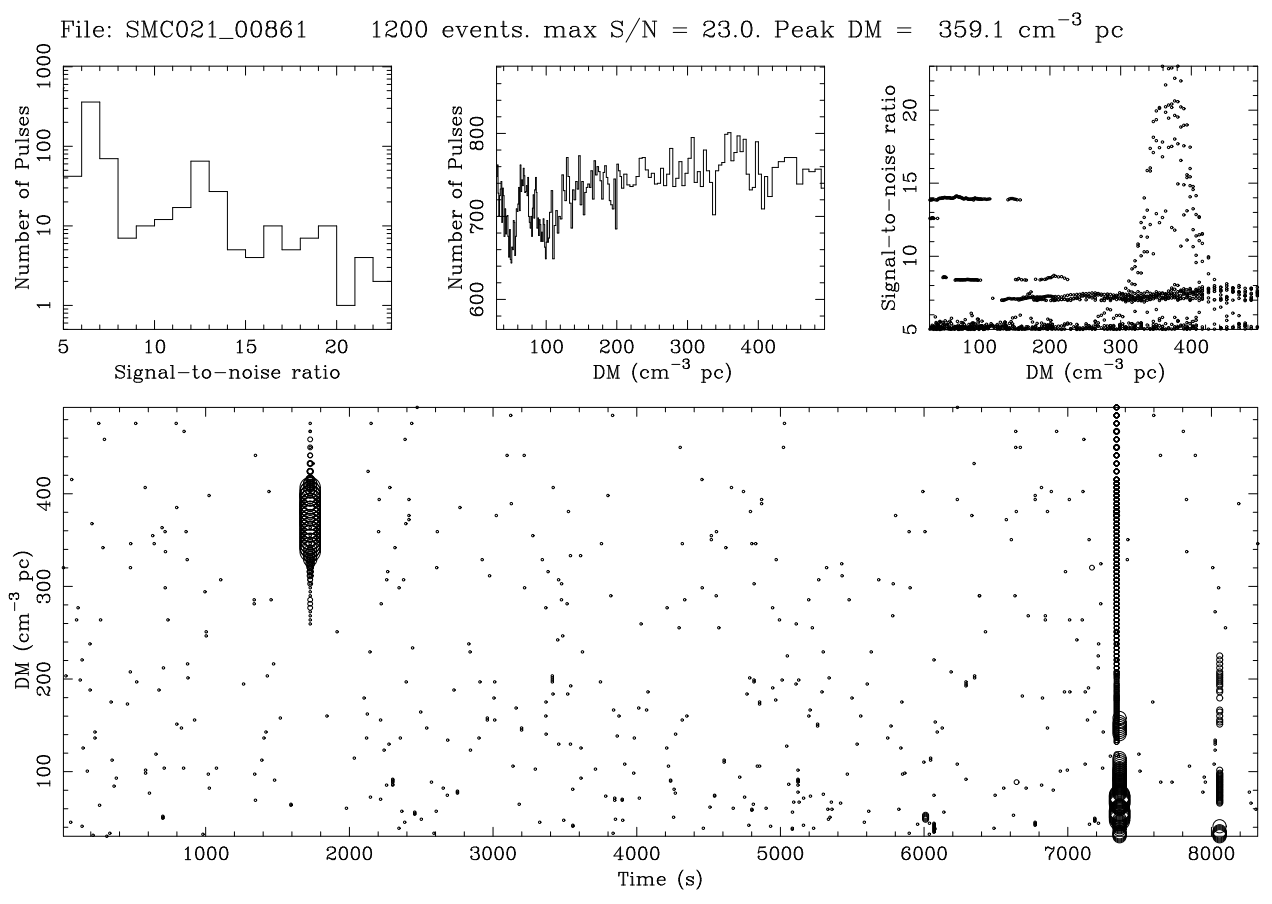

Figure 1.5: The output of a single-pulse search analysis done by Lorimer et al. (2007) and their detection of the Lorimer burst.

The Parkes multibeam receiver consists of 13 beams each with a beamwidth of $\sim 14^{\prime}$ and the Lorimer burst was seen in three beams of the Parkes multibeam. But these new bursts, named as 'perytons' were seen in all 13 beams. The peryton signal was seen to mimic the dispersive signal coming from an astrophysical pulse that has propagated through cold plasma. More such bursts were found in the reprocessing of the Parkes Multibeam (PM) pulsar survey data and the general consensus was that these bursts and the Lorimer burst might not be of astrophysical origin.

Keane et al. (2012) found another similar burst at $1.4 \mathrm{GHz}$ very close to the Galactic plane which was seen in only one beam and at a higher DM (= $\left.746 \mathrm{pc} \mathrm{cm}^{-3}\right)$. However, the confirmation that these bursts are astrophysical came only after the discovery of four bursts in the High Time Resolution Universe (HTRU) survey at $1.4 \mathrm{GHz}$ (Thornton et al., 2013). These highly dispersed pulses are now 
known as 'Fast Radio Bursts' (FRBs) and are labelled in YYMMDD format. A single dispersed burst would be classified as an FRB if it is bright, has a pulse width of a few ms and a DM greater than the modeled DM contribution along that line of sight. It should be noted that the NE2001 model has uncertainties up to $40 \%$ in certain directions. For example, in the case of FRB 010621 (Keane et al., 2012), $H_{\alpha}$ observations of the diffuse ionized gas along the line of sight showed that the pulse most likely resides in the Galaxy and the excess DM is caused by localized density enhancements along the line of sight (Bannister \& Madsen, 2014).

After the discovery of four bursts (FRB 110220, 110626, 110703, 120127) by Thornton et al. (2013), a number of pulsar archival surveys were searched for FRBs using the single-pulse search method up to a much higher DM. The typical values of DM for Galactic pulsars range from $2 \mathrm{pc} \mathrm{cm}^{-3}$ to $1800 \mathrm{pc} \mathrm{cm}^{-3}$, where high DM pulsars are near the center of our Galaxy (see Figure 1.6). Usually high latitude pulsar surveys are processed for DMs up to $300 \mathrm{pc} \mathrm{cm}^{-3}$ while searching for Galactic pulsars and RRATs. This is reasonable because there are about 600 pulsars that have DM values greater than $300 \mathrm{pc} \mathrm{cm}^{-3}$ and most of these pulsars are very close to the Galactic center with longitudes within $\pm 50^{\circ}$ and latitudes within $\pm 5^{\circ}$.

Soon after this, FRB 011025 was discovered in the processing of HTRU intermediate latitude survey. The fact that all FRBs were detected only in the data from the Parkes telescope raised some concern at that time. In 2014, FRB 121102 was discovered with the 305-m Arecibo telescope in the processing of the Pulsar Arecibo L-band Feed Array (PALFA) survey (Cordes et al., 2006; Lazarus et al., 2015) at 1.4 GHz. This burst was detected towards the Galactic anti-center at a DM of 


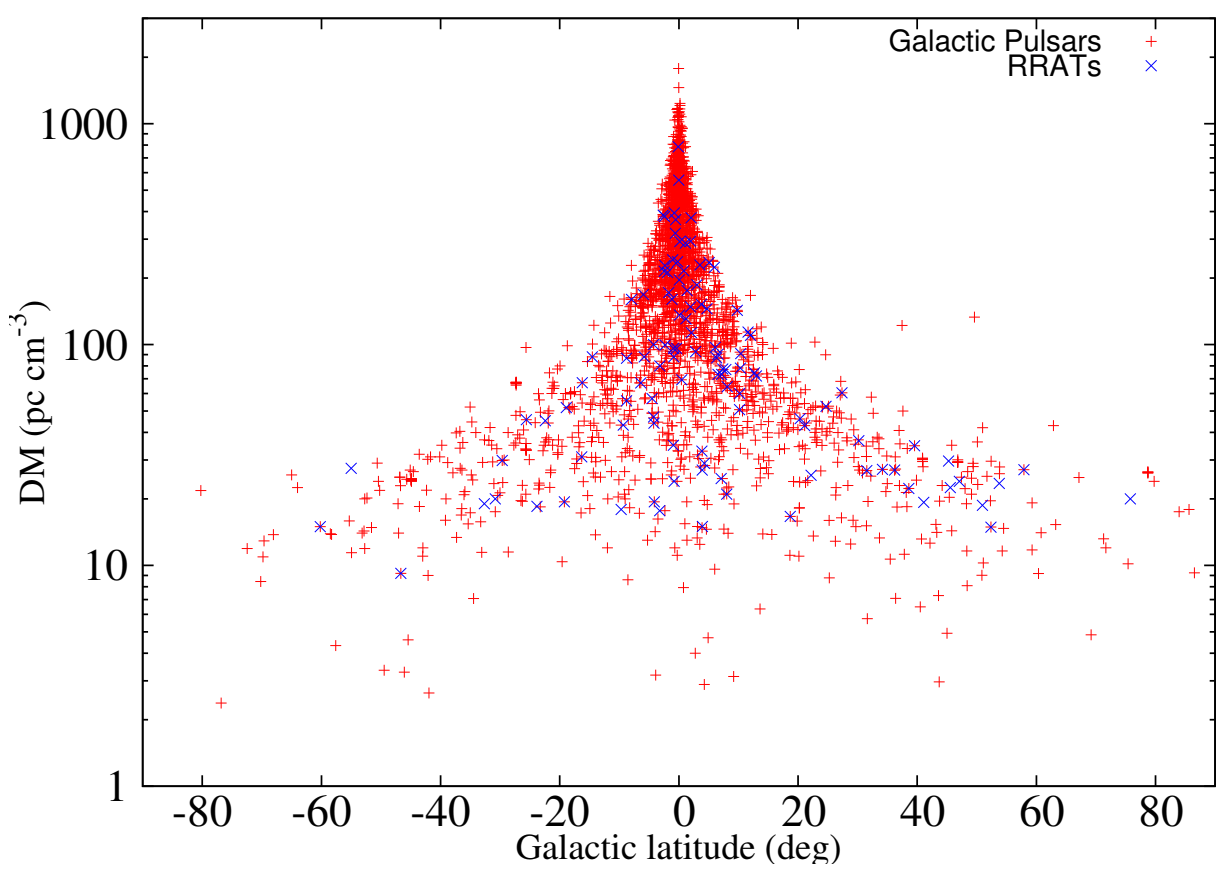

Figure 1.6: The DM-latitude dependence for Galactic pulsars and RRATs.

$557 \mathrm{pc} \mathrm{cm}^{-3}$ and this discovery cleared doubts about FRBs being some phenomena related to the Parkes telescope only.

The development of a real-time transient pipeline at the Parkes telescope using the multi-beam receiver led to the discovery of FRB 140514 at $1.4 \mathrm{GHz}$ (Petroff et al., 2015a). The authors were aiming to search the fields of previous FRBs to look for repeat events and FRB 140514 was detected in the same field as FRB 110220 but at a much lower DM. They argued that their proximity is due to sampling bias in their choice of observing location. After the real-time detection, follow-up observations were carried out with 12 telescopes observing from X-ray to radio wavelengths but they were unable to identify any afterglow-like variable multi-wavelength counterpart. When a SN or a long duration gamma-ray burst (GRB) goes off, a counterpart is detectable as an object of varying brightness. This type of afterglow is observable 
on time-scales ranging from hours (for a long GRB) to days (for typical SNe) and therefore this lack of detection suggested that FRBs are unlikely to be associated with long duration GRBs or SNe.

Another real-time discovery (FRB 131104) followed with the same pipeline in a targeted observation of the Carina dwarf spheroidal galaxy at $1.4 \mathrm{GHz}$ (Ravi et al., 2014). The dispersion measure of this FRB exceeded the maximum predicted line-of-sight Galactic contribution by a factor of 11 . Follow-up observations of 100 hours with the Parkes telescope did not detect any repeat events from this location. A $\gamma$-ray transient has recently been proposed to be associated with this FRB at the $3.2 \sigma$ to $3.4 \sigma$ confidence level with Swift (DeLaunay et al., 2016). This transient, J0644.5-5111, was seen for $\sim 380 \mathrm{~s}$. This is somewhat longer than most long-duration GRBs detected by Swift. They suggested that the $\gamma$-ray emission was generated by shocked relativistic plasma in a cosmological explosion, or in an accretion episode associated with a supermassive black hole. However, Shannon \& Ravi (2017) carried out observations of the field of FRB 131104 with the Australia Telescope Compact Array (ATCA) for 2.5 years beginning 3 days after the event and found no radio afterglow coincident with the $\gamma$-ray event. They argued that a true association is not significantly more likely than the probability of an unassociated occurrence.

Although FRBs were detected with two telescopes, all of the above mentioned FRBs were discovered between 1-2 GHz. In 2015, FRB 110523 was discovered with the Green Bank Telescope (GBT) in the search of an archival hydrogen intensity mapping survey carried out in the frequency range $700 \mathrm{MHz}$ to $900 \mathrm{MHz}$. This dis- 
covery strengthened the argument that FRBs are astrophysical phenomena (Masui et al., 2015).

Five additional FRBs (090625, 121002, 130626, 130628, 130729) were discovered in the processing of HTRU high latitude survey by Champion et al. (2016) with FRB 121002 detected at a DM of $1629 \mathrm{pc} \mathrm{cm}^{-3}$, the highest DM found so far. Efforts of searching for multi-wavelength counterparts and finding associations with host galaxy continued. In mid-2016, the discovery of FRB 150418 was reported with identification of a fading radio transient that lasted over the course of six days after the FRB event (Keane et al., 2016). The authors used it to identify a host galaxy at a redshift of 0.492. But further observations (Williams \& Berger, 2016; Vedantham et al., 2016) suggested that the observed variable radio emission is instead due to an active galactic nuclei (AGN) activity and are unrelated to FRB 150418.

In 2015, FRB 150807 was detected with the Parkes telescope while timing observations of a millisecond pulsar were being carried out. This FRB has a DM of $266.5 \mathrm{pc} \mathrm{cm}^{-3}$, which is the lowest DM for an FRB found so far. This FRB is very bright $(\sim 12 \mathrm{Jy})$ and is also linearly polarized.

Very recently, Caleb et al. (2017) discovered three FRBs at $843 \mathrm{MHz}$ in real-time with the UTMOST array at the Molonglo Observatory Synthesis Telescope in Australia. These are the first FRBs discovered with an interferometer (160317, 160410, 160608). UTMOST array consists of an east-west aligned cylindrical paraboloid divided into two 'arms', each 11.6-m wide and 778-m long. They performed a 180-day survey of the Southern sky and followed up at these locations for 100 hours which resulted in no repeat events. 
Petroff et al. (2017) reported another real-time discovery (FRB 150215) with Parkes at a DM of $1105 \mathrm{pc} \mathrm{cm}^{-3}$ and is found to be $43 \%$ linearly polarized. The Australian SKA Pathfinder (ASKAP) discovered a very bright (22 Jy) FRB 170107 in the pilot survey at a DM of $610 \mathrm{pc} \mathrm{cm}^{-3}$ (Bannister et al., 2017).

Each one of the 23 FRB discoveries mentioned above is important to help us understand their properties, possible progenitors, and the FRB population as a whole.

Along with these discoveries, in 2015, the source of the millisecond-duration transients of terrestrial origin or the perytons was identified. Around 25 perytons were recorded since 2011 (Burke-Spolaor et al., 2011; Kocz et al., 2012; Bagchi et al., 2012; Saint-Hilaire et al., 2014) and were observed only during office hours and on weekdays. This suggested that they might be a form of human-generated RFI. Petroff et al. (2015b) found out that a peryton can be generated at $1.4 \mathrm{GHz}$ when a microwave oven door is opened prematurely and the telescope is at an appropriate relative angle.

Inside a microwave, the magnetron pulls electrons from the power source and then uses magnets to rotate them around inside a vacuum. This generates microwaves and the magnetron power cycle can be set to a duration depending on the manufacturer. If the microwave door is opened before this cycle is complete then the radio emission escaping from microwave ovens during this phase can generate a peryton signal. They performed specific tests to verify this and detected three perytons on three separate days. By comparing properties of perytons and FRBs, Petroff et al. (2015b) demonstrated that perytons are strongly clustered in DM and 

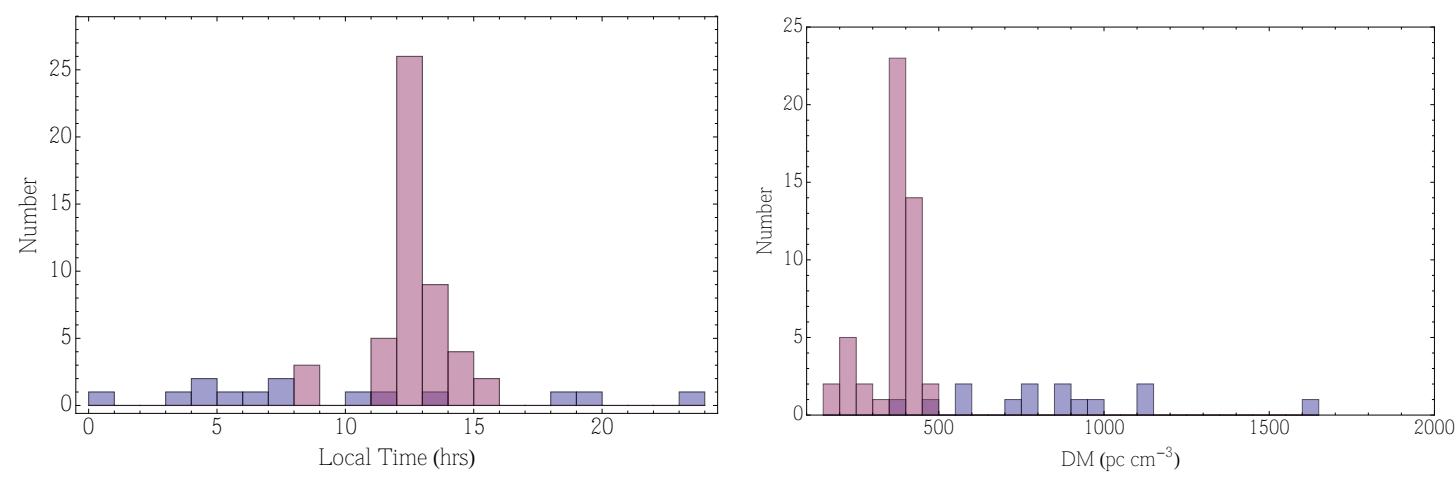

Figure 1.7: The FRB (blue) and perytons (red) distributions as a function of local time at the Parkes facility and as a function of DM obtained from Petroff et al. (2015b).

time of the day unlike FRBs which are uniformly distributed (see Figure 1.7). For example, FRB 010724 was detected at 4 a.m. From these observational differences, they concluded that FRBs and perytons arise from different origins and that FRBs are indeed astrophysical in origin.

\subsubsection{Repeating FRB}

All of the FRBs have been searched for repeat bursts but most of the efforts were unsuccessful. Some of the radio follow-ups are listed in Table 1.1. Initial followup observations of FRB 121102 were carried out for a few hours with no detection (Spitler et al., 2014). In 2015, this FRB was extensively observed with the Arecibo telescope around the best known sky position. This effort resulted in ten additional bursts (Spitler et al., 2016) in three hours of observations, confirming it as the only repeating FRB source observed so far.

These detections motivated further follow-up multi-wavelength campaigns and Scholz et al. (2016) found five bursts with the GBT at 2-GHz, and one at $1.4 \mathrm{GHz}$ 
Table 1.1: List of FRB follow-up observations.

\begin{tabular}{lcc}
\hline FRB & $\begin{array}{c}\text { Total duration } \\
(\text { hr })\end{array}$ & Reference \\
\hline 010724 & $>200$ & (Lorimer et al., 2007) \\
090625 & $\sim 34$ & (Petroff et al., 2015) \\
110220 & $\sim 2$ & (Petroff et al., 2015) \\
110626 & $\sim 11$ & (Petroff et al., 2015) \\
110703 & $\sim 10$ & (Petroff et al., 2015) \\
120127 & $\sim 6$ & (Petroff et al., 2015) \\
131104 & $\sim 100$ & (Shannon \& Ravi, 2017) \\
150807 & $\sim 215$ & (Ravi et al., 2016) \\
121002 & $\sim 10$ & (Petroff et al., 2015) \\
140514 & $\sim 19$ & (Petroff et al., 2015) \\
130626 & $\sim 10$ & (Petroff et al., 2015) \\
130628 & $\sim 9$ & (Petroff et al., 2015) \\
\hline
\end{tabular}

with Arecibo. All of these 17 bursts have a same DM that is consistent with that of FRB 121102. This provided a strong evidence that the bursts were originating from the same source.

The bursts appear to cluster in time but no underlying periodicity has been detected yet. The peak flux densities were in the range of $0.02-0.3 \mathrm{Jy}$ at 1.4 $\mathrm{GHz}$, suggesting that weaker bursts are also produced, preferably at a higher rate. No evidence for scatter broadening or polarization is seen in any of these bursts, however, after fitting a power-law model $\left(S_{\nu} \propto \nu^{-\alpha}\right)$ to burst spectra, the spectral index was found to range from -10 to +14 . This varying spectral index could be intrinsic to the source or due to propagation effects as suggested by Scholz et al. (2016).

The repeating bursts from FRB 121102 led to targeted interferometric localization efforts. In 2016, 83 hours of simultaneous observations with the Karl Jansky Very Large Array (VLA) and the Arecibo telescope, spanned over six months, de- 
tected nine bursts in the $2.5-3.5 \mathrm{GHz}$ band and reported the first sub-arcsecond localization (Chatterjee et al., 2017). The beamformed single-pulse search and millisecond imaging resulted in detection of a persistent variable radio counterpart with a flux density of $180 \mu \mathrm{Jy}$ (Marcote et al., 2017) and a star-forming host galaxy at a redshift of 0.19 (Tendulkar et al., 2017). The host galaxy is very small with a stellar mass of $(4-7) \times 10^{7} \mathrm{M}_{\odot}$. The European very-long-baseline interferometric (VLBI) network at $5 \mathrm{GHz}$ detected 4 bursts and showed that the projected separation between the persistent radio source and FRB is less than $40 \mathrm{pc}$, suggesting a strong physical link.

\subsubsection{Dispersion measure}

All of the 23 FRBs mentioned above have DMs exceeding the Galactic DM contribution by a factor between 1.5 to 11 . Although the NE2001 model could be uncertain up to $40 \%$ along certain lines of sight, these events can still be distinguished from the other radio transients. Figure 1.8 is a similar plot as Figure 1.6 showing the DM distribution as a function of latitude but for all radio transients. The pulsars in the LMC and SMC can be clearly seen above the Galactic pulsar population with additional DM coming from the electrons between LMC, SMC and the Earth and from the LMC, SMC itself.

However, FRBs have no correlation between DM and latitude dependence indicating that the total DM includes a large extragalactic component. Ionized gas in galaxies and in the IGM are therefore plausible sources for this extragalactic part 
of the DM. The measured DM follows as $\nu^{-2}$ which shows that the electromagnetic signal passes through the cold plasma. Some authors (e.g., Loeb et al., 2014) have suggested that the excess DM might arise from flaring stars. But the plasma near the surface is not cold and the resulting signal would not follow the $\nu^{-2}$ behavior which is seen for all FRBs. If the free electron distribution in the host galaxy is similar to that in our Galaxy, then a host DM can be estimated. For $z \geq 0.2$, the DM contribution from the IGM is expected to dominate for FRBs at higher latitudes (Ioka, 2003; Inoue et al., 2004). Then the IGM contribution can be computed by subtracting the host DM and DM due to the MW from the total measured DM. And the DM due to the IGM depends on the redshift which can be used to infer a redshift of an FRB The inferred redshifts for the known FRBs range from 0.19-2.2. Only one independent measurement has been done to date for FRB 121102 at $z=0.193$ (Tendulkar et al., 2017). Cordes et al. (2016) argue that the redshifts can be significantly overestimated if they are based on the assumption that the extragalactic portion of DM is dominated by the IGM as it is also possible that the host galaxy dominates the extragalactic DM contribution.

\subsubsection{Scattering}

In the case of FRBs, the Galactic ISM, IGM, host ISM, and intervening galaxy or galaxy clusters all cause turbulence-induced scatter broadening. The scattering in FRBs can be used to study turbulence in the IGM and in other galaxies. FRB scattering timescales are within the range spanned by pulsars but are many orders 


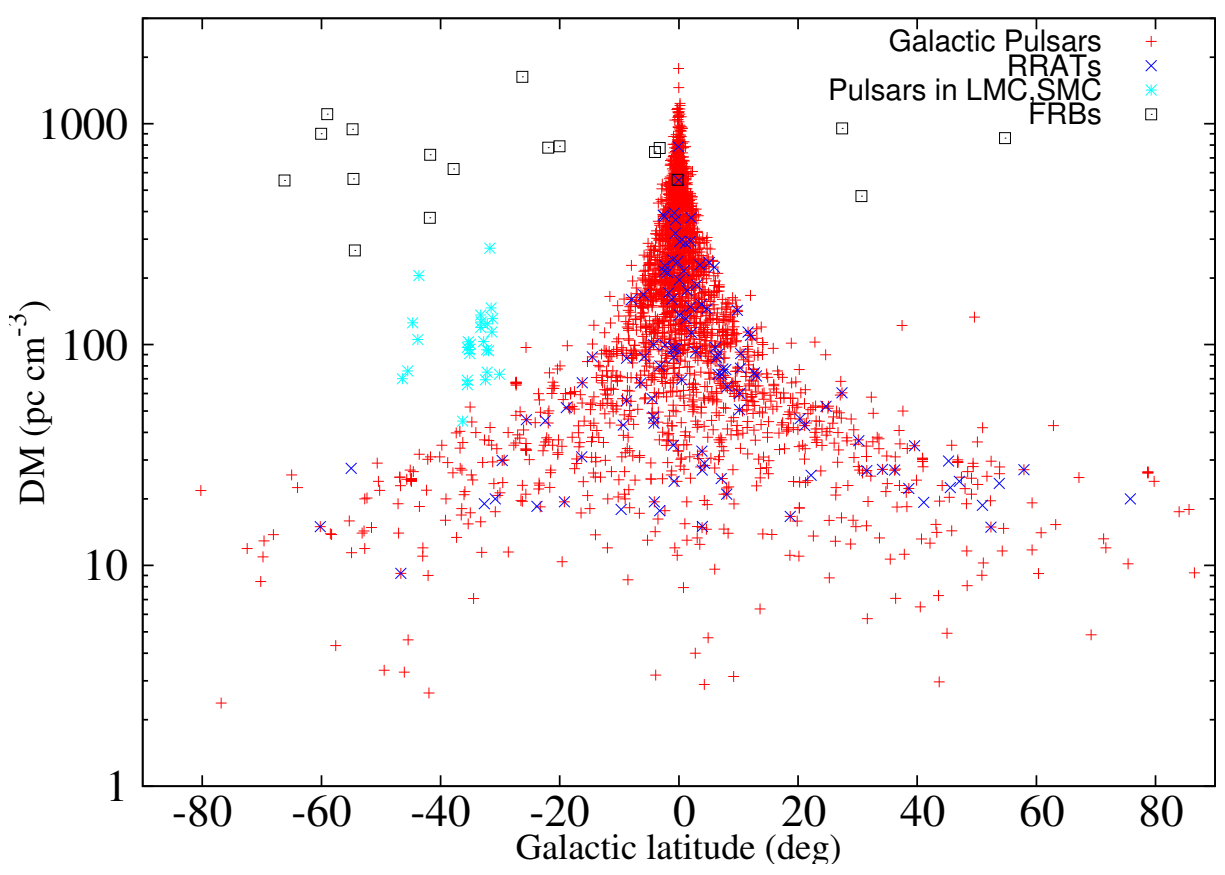

Figure 1.8: The DM latitude dependence for all radio transients.

of magnitude larger than pulsars at similar Galactic latitudes, as seen in Figure 1.9.

Nine out of 23 known FRB sources show asymmetric pulse broadening caused by scattering from small-scale electron-density variations. The left panel of Figure 1.10 shows FRB profiles with no scattering. FRBs with scattering are shown in the right panel. The measured pulse broadening for FRBs is much larger than expected from the Milky Way for the directions of FRBs but the broadening is smaller than would be expected from the $\tau-\mathrm{DM}$ relation for Galactic pulsars having the same DM. The Milky Way can only account for scattering timescales less than a microsecond (Figure 1.9). Observations of scattering along extragalactic lines of sight by Lazio et al. (2008) and theoretical calculations by Macquart \& Koay (2013) suggest that scattering in the IGM is several orders of magnitude lower than in the ISM. If the IGM contributed enough to the scattering then that would require a 


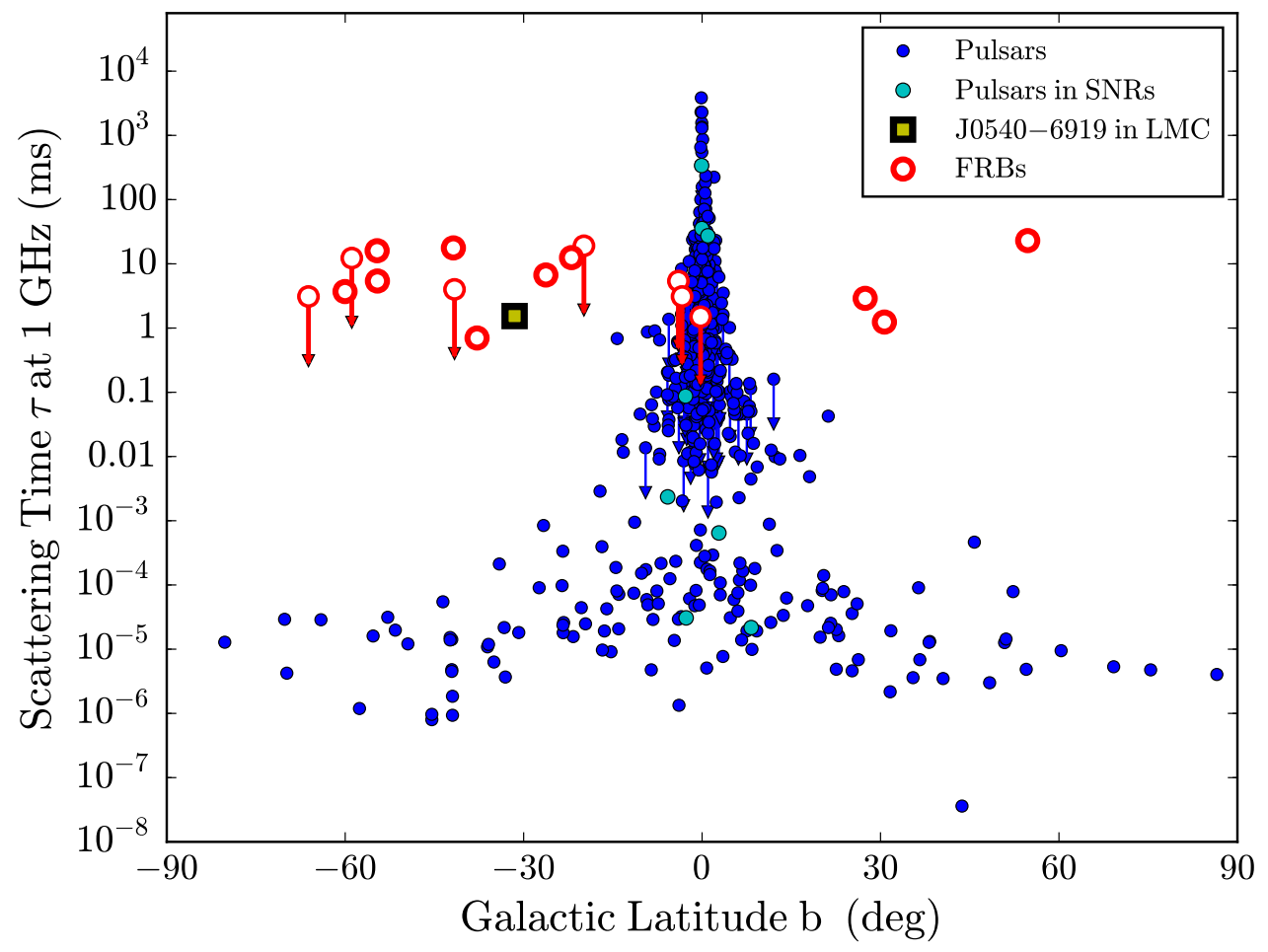

Figure 1.9: The scattering timescales for pulsars and FRBs. This figure is obtained from Cordes et al. (2016). 

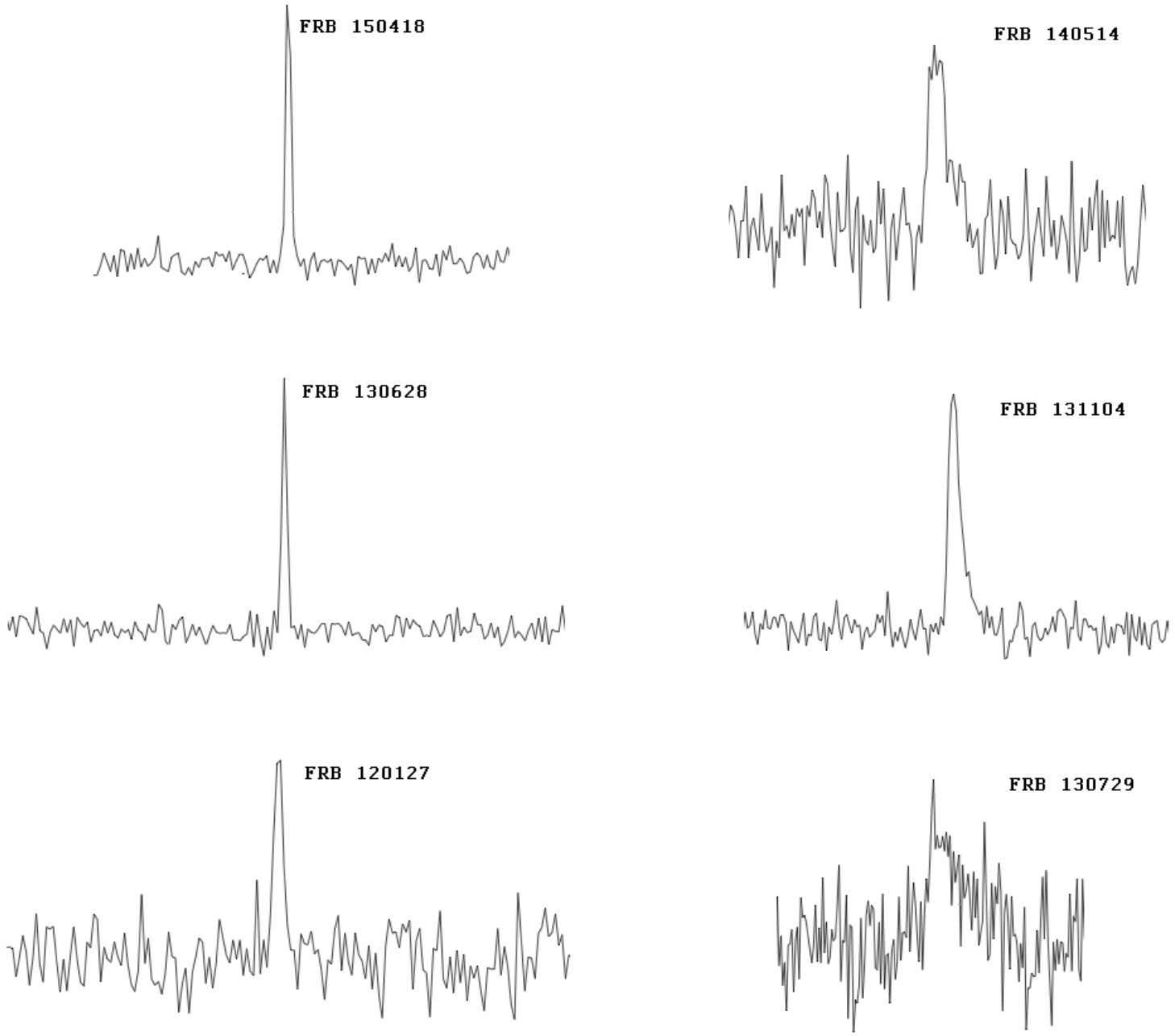

Figure 1.10: FRBs with no scattering are shown in the left panel and FRBs with scattering are shown in the right panel. The horizontal time scale is $60 \mathrm{~ms}$ for each profile. 
level of turbulence an order of magnitude higher than encountered at the Galactic center. The low density diffuse IGM cannot support such density fluctuations and therefore cannot reproduce the measured scattering for FRBs. The scattering contribution from intervening galaxies and galaxy clusters near the line of sight is not an important effect because the probability of this alignment is very low. Note the probability of an intervening source within $z<1$ is less than 0.05 (Roeder \& Verreault, 1969). This makes the contribution from the host galaxy the most prominent source of scattering in FRBs. This assumes that the ISM in the host galaxy has the same properties as in the MW at all redshifts. But at higher redshifts, observations suggest that the ISM in those galaxies was more turbulent and dense $(z \sim 2)$ (Xu \& Zhang, 2016). Overall, the scattering timescale provides valuable insights into the IGM turbulence concerning the detailed structure of density and magnetic field of the IGM (Xu \& Zhang, 2016).

\subsubsection{Polarization}

For astrophysical sources, the electric field vectors are plane waves. For a wave traveling in the $z$ direction at time $t$ with angular frequency $\omega$ and amplitude $E_{0}$, the electric field is

$$
\vec{E}=E_{0}\left[\hat{a} e^{\left(i k_{+} z-\omega t\right)}+\hat{b} e^{\left(i k_{-} z-\omega t\right)}\right]
$$

The wave traces out an ellipse in the electric field plane with major and minor axes $\hat{a}$ and $\hat{b}$. If the radio signal is linearly polarized then the wave vector in the direction

$\hat{a}\left(k_{+}\right)$is equal to the wave vector along $\hat{b}\left(k_{-}\right)$. However, if the wave is traveling 
through a magnetic field then this condition does not hold since the angle between the electric field vector and the plane of incidence (polarization angle, $\Psi$ ) rotates as the wave propagates through plasma. This effect is known as the Faraday rotation. The rotation of the angle of linear polarization is called rotation measure (RM) and it is proportional to the line of sight component of magnetic field $\left(B_{\|}\right)$weighted by electron density $n_{\mathrm{e}}$. We define

$$
\mathrm{RM}=\frac{e^{3}}{2 \pi m_{e}^{2} c^{4}} \int_{0}^{d} n_{e} B_{\|} d l
$$

where $e, m_{\mathrm{e}}$ are electron charge and mass respectively, $c$ is the speed of light, $d$ is the distance to the source. RM is measured in units of $\mathrm{rad} \mathrm{m}^{-2}$. The variation in polarization angle $(\Delta \Psi)$ is obtained over a wide frequency range

$$
\Delta \Psi=\operatorname{RM} \lambda^{2}
$$

The measurement of RM and DM gives the average magnetic field strength along the line of sight:

$$
\left\langle B_{\|}\right\rangle=\frac{\int_{0}^{d} n_{e} B_{\|} d l}{\int_{0}^{d} n_{e} d l} .
$$

Here the magnetic field strength is in $\mu \mathrm{G}$. This relation works very well for Galactic pulsars. However, in the case of FRBs, it cannot be used directly to estimate the intergalactic magnetic field as FRBs are cosmological sources. The RM and DM have different redshift dependencies. As noted in Akahori et al. (2016), the DM at cosmological scale is dominated by contributions from the warm-hot intergalactic 
medium (WHIM) in filaments and from the gas in voids and the RM is induced mostly by the hot medium in galaxy clusters, with only a fraction of it produced in the WHIM. They modify Equation 1.12 as

$$
\left\langle B_{\|}\right\rangle=\frac{\langle 1+z\rangle_{\mathrm{WHIM}}}{f_{\mathrm{DM}, \mathrm{WHIM}}} \frac{\int_{0}^{d} n_{e} B_{\|} d l}{K \int_{0}^{d} n_{e} d l} .
$$

Here $f_{\mathrm{DM}, \mathrm{WHIM}}$ is the fraction of the total DM due to the WHIM and can be evaluated for a given cosmology model using the DM of an FRB. With this simple modification, the density-weighted line-of-sight magnetic field strength of the intergalactic medium in filaments of the large-scale structure can be reconstructed.

To constrain the emission mechanism, we need polarization information of FRBs. As of now, only a few FRBs have shown polarization. FRBs 140514 is found to be $20 \%$ circularly polarized but no linear polarization was detected and hence the RM could not be determined (Petroff et al., 2015a). The authors concluded that this polarization is intrinsic to the FRB and suggested that if FRBs emit coherently then there would have been intrinsic linear polarization but it may have been depolarized by Faraday rotation caused by passing through strong magnetic fields and/or highdensity environments. Faraday rotation was measured for FRB 110523 which was detected in the archival data from GBT (Masui et al., 2015). This FRB is $44 \%$ linearly polarized with a best-fitting $\mathrm{RM}$ of $-186.1 \mathrm{rad} \mathrm{m}^{-2}$. The average line-ofsight component of the magnetic field is $0.38 \mu \mathrm{G}$. The authors claimed that this magnetization is local to the FRB source as the contributions to the RM along this line-of-sight within the Milky Way and from the IGM are small. FRB 150418 
was found to be only $8.5 \%$ linearly polarized with no circular polarization (Keane et al., 2016). The authors determined RM of $36.0 \mathrm{rad} \mathrm{m}^{-2}$ but mention that this measurement is not very precise since the linear polarization is very low. They place an upper limit on the electron weighted IGM magnetic field strength of $\sim 0.4 \mu \mathrm{G}$. Ravi et al. (2016) found FRB 150807 to be 80\% linearly polarized giving a RM of $12.0 \mathrm{rad} \mathrm{m} \mathrm{m}^{-2}$. They constrained the average line-of-sight magnetic field to be $\left\langle 21\left(1+z_{\text {mean }}\right)\right\rangle \mathrm{nG}$, where $z_{\text {mean }}$ is the mean redshift of the intervening electron density distribution.

Although currently we have only a few Faraday rotation measurements, the development of polarization triggering modes along with the real-time detection system at Parkes and at other telescopes will enable us to get polarization information for all detections. The measurement of RM can give useful insights into the local environment around the FRB sources.

\subsubsection{Progenitor models}

The physical nature and the progenitors of FRBs still remain a mystery. Although a large number of theories exist to explain their origin, none of them have been conclusively proven correct from the observations. The proposed model should be consistent with the very high event rate of FRBs and should explain the large DMs, timescales, and brightnesses as well. The best estimate for FRB all sky event rate is $3.3_{-2.2}^{+3.7} \times 10^{3}$ events per day per sky (see Chapter 3 ). The currently proposed models involve sources which are either cataclysmic in nature (i.e. producing only 
one burst), or capable of producing multiple bursts.

The cataclysmic models include merging or collapsing of compact objects. Falcke \& Rezzolla (2014) propose that a neutron star created above the theoretical mass limit (supra-massive neutron star), would be supported by centrifugal force for an extended period of time, until the star is spun down enough due to various torques, the most dominant of which may be the magnetic dipole spin down so that centrifugal force can no longer support the star. At this epoch, the neutron star would collapse into a black hole. The magnetosphere is the only part of the neutron star which will not disappear in the collapse as it is well outside the neutron star. The entire magnetic field should in principle detach and reconnect outside the event horizon. This results in large currents producing strong shock and intense electromagnetic emission, which is bright enough to explain the observed fast radio bursts. The characteristic timescale for such an event is less than a millisecond. Falcke \& Rezzolla (2014) also argue that only a few percent of the neutron stars are needed to be supramassive in order to explain the observed FRB rate.

Some authors have discussed the binary neutron star mergers as a possible origin of FRBs (Piro, 2012; Totani, 2013; Wang et al., 2016) as both rates are consistent. At the time of coalescence, the magnetic fields of neutron stars are synchronized to binary rotation and the radiation mechanism due to magnetic braking seems favorable to explain the millisecond duration of FRBs before merging into a black hole (Totani, 2013). In a NS binary in which one NS is highly magnetized compared to the other one, the magnetic torques spin up the magnetized NS draining the angular momentum from the binary and accelerating the inspiral. An 
electromotive force induced on one NS accelerates electrons to an ultra-relativistic speed and the resultant coherent curvature radiation from these electrons moving along magnetic field lines in the magnetosphere of the other NS is responsible for the observed FRB signal. The coalescence would leave behind a rapidly rotating black hole which could be a possible source of gravitational wave event (Piro, 2012; Wang et al., 2016).

FRBs could be produced in another scenario such as merging of white dwarfs (WD) as suggested by Kashiyama et al. (2013) in which the coherent emission is produced from the polar region of a massive rapidly rotating and magnetized WD formed after the merger. A SN Ia is one possible counterpart in this model but the lack of any such associations from current observations question this model for FRBs. Other interesting models could also explain a sub-class of FRBs. These include NS-BH mergers (Mingarelli et al., 2015), BH-BH mergers (Zhang, 2016; Liebling \& Palenzuela, 2016), the discharging of charged black holes (Liu et al., 2016), and evaporating BHs (Keane et al., 2012).

The discovery of a repeating FRB, however, rules out the cataclysmic models at least for this FRB or for a subset of FRBs. In this scenario, if FRBs are observed for long enough time with more sensitivity, either all or some of them might be seen to repeat. However, this is still under debate and there is no robust evidence yet for one or multiple FRB progenitors, if there are any, from observations. We cannot rule out the cataclysmic models completely as of now, but we can place strong constraints on what type of progenitors could be producing this type of emission. Most of the known FRBs have been followed up in radio or at other wavelengths as of 
now. However, not all non-detections have been reported and hence it is difficult to conclude if any other FRB should have been seen to repeat. It could be possible that the repeater belongs to a population of sources at a different evolution phase from other FRBs. But we see no distinction in the observed properties of repeater and other bursts. We still need more FRBs to support or refute this kind of hypothesis. It should also be noted that as mentioned earlier, the sensitivity of Parkes is less than that of Arecibo so there is a possibility that they all repeat but might be below our detection threshold. In fact, the faintest Arecibo detections have flux densities an order of magnitude smaller than those of the faintest Parkes FRBs. In this case we are only detecting the brightest pulse from the source and other pulses remain undetected.

The narrowest pulses from FRBs constrain the emission region size based on the light travel time. FRB 150807 has the narrowest pulse of $0.35 \mathrm{~ms}$. This gives us an emission region size of $\sim 105 \mathrm{~km}$ which is comparable to sizes of compact objects. Neutron stars also generate coherent emission from a small emission region and produce large amount of energy similar to observed from FRBs. Based on these current observations, we only discuss a handful of the most promising models that could explain the repeatable origin of FRBs. Most of these models include some relation with NS emission.

Cordes \& Wasserman (2016) propose that FRBs are associated with bright pulses from extragalactic pulsars. Some pulsars (for example, the Crab pulsar) are known to emit giant pulses and the NS formation rate in a Hubble volume is comparable to the $\mathrm{FRB}$ rate. In this case, the emission will be rotationally driven. If 
pulsars at distances within $100 \mathrm{Mpc}$ emit such bright pulses then this population of pulsars within this distance may be the source of FRBs. However, the giant pulses emitted from the Crab are not typical of the pulsar population as a whole. Taking this into account, the authors argue that the giant pulses from extragalctic pulsars might be magnified through gravitational lensing of individual stars. Although the energetics and rate arguments are consistent with the properties of FRBs, it should be noted that the probability of seeing a repeat pulse from such an object is extremely low on human timescales (Cordes \& Wasserman, 2016).

Pen \& Connor (2015) suggest that FRBs are bursts from extragalactic but non-cosmological young pulsars and magnetars. Young neutron stars are energetic and are embedded in a supernova remnant (SNR). If the number of young pulsars is proportional to the core-collapse supernova rate and each pulsar emits a giant pulse every 100 days or so then the FRB rate is consistent within a local volume of about $200 \mathrm{Mpc}$ (Connor et al., 2016). Within a few hundred years of a core-collapse SN, the ejecta is confined within one parsec. This region could contribute to the excess DM. The magnetar model is based on the observed properties (polarization, DM, scattering) of the radio loud magnetar in our Galactic center. Pen \& Connor (2015) suggest that such galactic center magnetars within a few hundred megaparsecs could be the source of FRBs in which the emission will be magnetically powered and the excess DM could come from the overdense region near the host galaxy's galactic center. However, it should be noted that the population of such magnetars is smaller than that of young pulsars (Kaspi \& Beloborodov, 2017).

The soft gamma repeaters (SGRs) are a type of magnetars which exhibit gi- 
ant flares due to relativistic explosions in the magnetospheres (Lyutikov, 2006). In addition, they also produce non-thermal radio emission or hyperflares as suggested by Lyutikov (2002). Popov \& Postnov (2013) showed that the energetics of such hyperflares are consistent with that of FRBs. However, no radio emission was detected at Parkes during SGR 1806-20 giant flare (Tendulkar et al., 2016) which provides arguments against the magnetar association. If indeed FRBs are related to magnetar giant flares then we should expect to detect prompt high-energy afterglow and a very bright optical flash (Lyutikov \& Lorimer, 2016).

Both of the above supergiant pulse models invokes nearby galaxies that are not at cosmological distances and therefore are challenged by the fact that the repeater is located in a host galaxy at redshift $z=0.193$. Initial observations of the repeater did not show extended emission in radio, IR or in $H_{\alpha}$ which suggested that this FRB is not associated with an HII region or a pulsar wind nebula as the luminosity is very high and that the radio persistent source is unlikely to be an AGN since no optical or X-ray signatures are seen.

After the localization of FRB 121102 and based on the observed properties of this FRB, some of the above models can be tested. Murase et al. (2016) suggested that if FRBs are indeed powered by a young NS then the counterparts of FRB can be observed as quasi-radio nebular sources. The quasi-steady counterpart of FRB 121102 is broadly consistent with theoretical predictions. The authors consider young NSs including magnetars as the source of FRBs and calculate associated nebula emission of magnetar and pulsar-driven supernovae including super-luminous supernovae (SLSNe). Metzger et al. (2017) propose that the repeated bursts from 
FRB121102 originate from a young magnetar remnant embedded within a young hydrogen-poor SNR since the properties of the host galaxy of FRB 121102 are consistent with those of long-duration GRBs and hydrogen poor SLSNe. The host galaxy of FRB 121102 is a low metallicity, low mass galaxy in which SLSNe and long-duration GRBs are common (e.g., Stanek et al., 2006). Further studies of this FRB will tell us more about the neighbourhood of this galaxy and if it has any companions.

\subsubsection{FRBs as cosmological tools}

The standard cosmological model or the $\Lambda$-Cold Dark Matter $(\Lambda-\mathrm{CDM})$ model attempts to explain the existence and structure of the cosmic microwave background, the large-scale structure in the distribution of galaxies, abundances of hydrogen, helium, and also the accelerating expansion of the universe. The letter $\Lambda$ represents the cosmological constant associated with the dark energy which is used to explain the accelerating expansion of the Universe. The measurement from the Planck Collaboration et al. (2016) estimates the fraction of the total energy density of our universe that is dark energy to be $\sim 69 \%$. In addition, the dark matter component, supposed to be consisting of the hypothetical particles called as weakly interacting massive particles (WIMPs) accounts for the gravitational effects observed in large-scale structures and is currently estimated to constitute about $\sim 26 \%$ of the total energy density of the universe. The remaining $\sim 5 \%$ comprises all ordinary matter that is made of protons and neutrons that make up atoms and we refer 


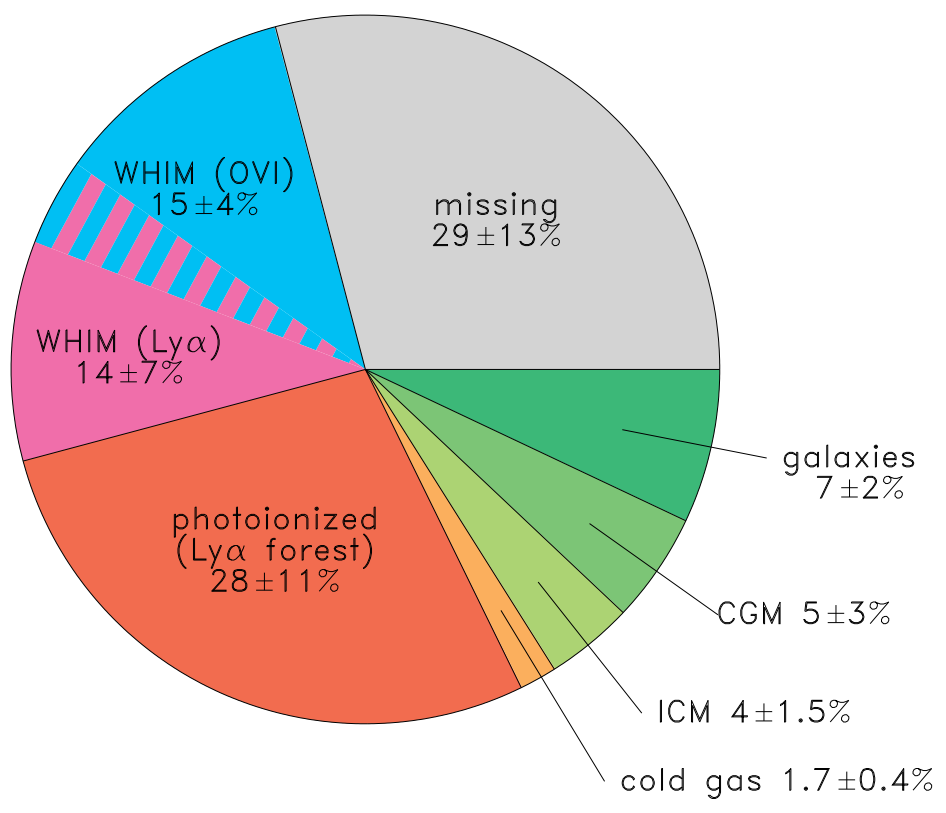

Figure 1.11: Current observational measurements of the low redshift baryon census obtained from Shull et al. (2012).

to it as the baryonic matter (detectable matter). Observations tell us that all the matter in stars, gas, and dust between galaxies is not enough to account for all the baryon content. In the low-redshift IGM, approximately $30 \%$ of the baryons reside in the warm intergalactic phase observed in photoionized diffuse Ly $\alpha$ absorption and $\sim 15 \%$ in the hot gas traced by OVI absorbers (see Figure 1.11). An additional $5 \%$ may reside in circumgalactic gas (material in galaxy halos), $7 \%$ in galaxies, and $4 \%$ in clusters (Nicastro et al., 2008; Shull et al., 2012). This leaves a large fraction $(\sim 29 \%)$ that is still not accounted for and is referred to as 'missing baryons'. Finding these missing baryons is crucial to validate our standard cosmological model. Most of the missing baryons are believed to be residing in the warm-hot IGM (WHIM) where the temperatures are high $\left(10^{5}-10^{7} \mathrm{~K}\right)$ and density is low. Therefore no significant absorption or emission is seen posing difficulties in detecting baryons using the 


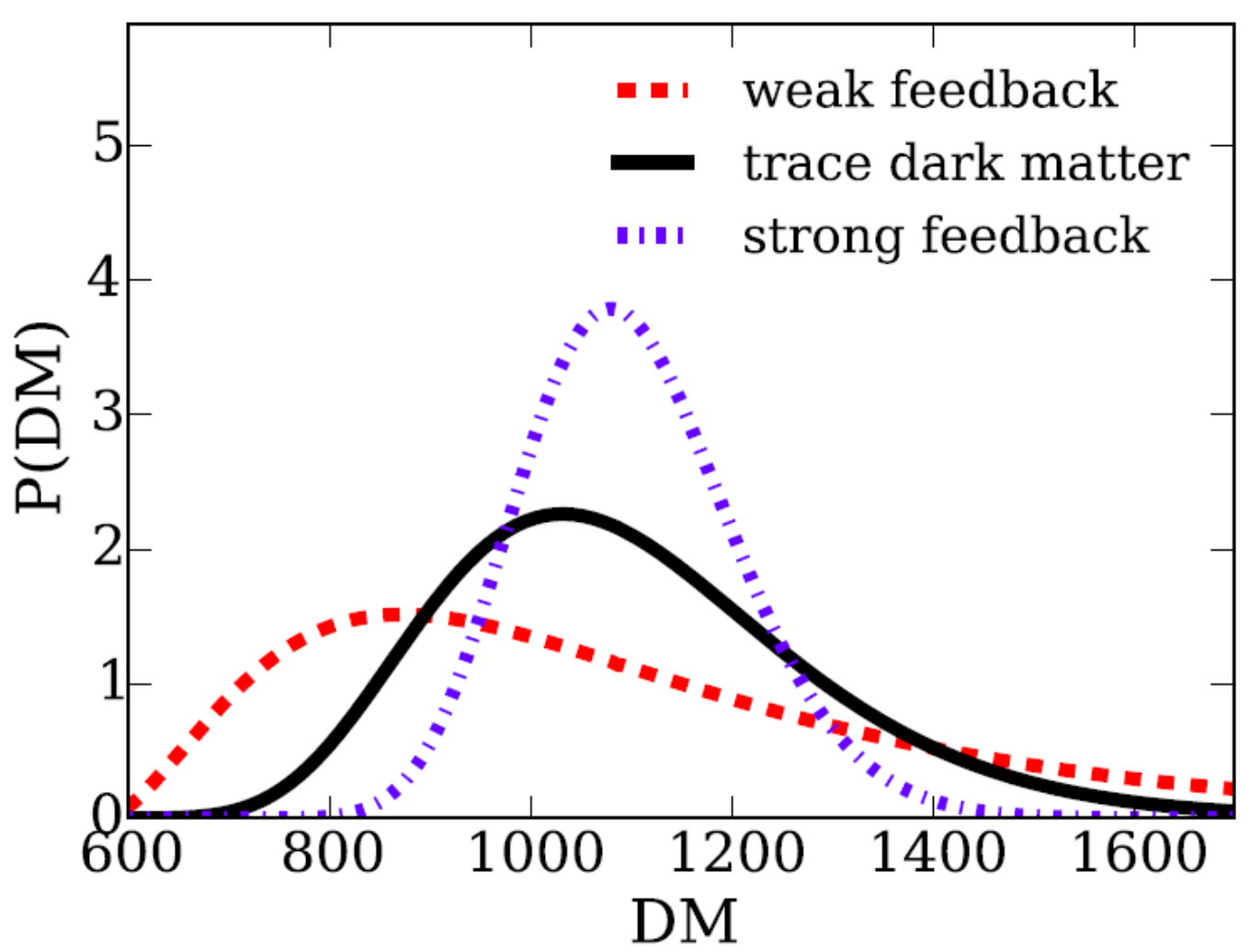

Figure 1.12: Dispersion in DM for sources at $z \sim 1$. Different DM distributions are obtained for FRBs at the same $z$ depending on how far the baryons are stretching the halos of the galaxies along the line of sight. This figure is obtained from McQuinn (2014).

conventional spectral line diagnostics. If we could measure the redshifts of FRBs independently then FRBs would be capable of detecting every single ionized baryon along the line of sight and thus could be direct detection of the missing baryons. In the low-redshift universe $(z<3)$, the helium is fully ionized (McQuinn et al., 2009) and homogeneously distributed such that $n_{\mathrm{e}} \propto \Omega_{\mathrm{b}}(1+z)^{3}$ (Ioka, 2003) where $\Omega_{\mathrm{b}}$ is the baryon density. And $\mathrm{DM}_{\mathrm{IGM}} \propto n_{\mathrm{e}}$, hence the DM- $z$ relation can be used to estimate the baryon density along the lines of sight of FRBs. Also, most of the dark matter is believed to reside in galactic halos, however, much less than half of the baryons are observed to lie within these halos (McQuinn, 2014). Using the 
DM- $z$ relation for FRBs as a cosmic ruler is not quite straightforward as the DM of an FRB varies as it travels through the IGM. McQuinn (2014) calculated the probability distribution functions (PDFs) of DMs for FRBs with $z \sim 1$ and showed that the dispersion in DM depends strongly on the strength of the feedback. The feedback is provided through stellar winds, supernovae, or AGN activity. Figure 1.12 is obtained from McQuinn (2014) and it shows dispersion in DMs for FRBs at the same redshift. The black solid line refers to a radius up to which the dark matter halo is traced. A strong feedback scenario refers to baryons extending out to a larger radius beyond the dark matter halo radius and the PDF of DMs is more concentrated giving a Gaussian distribution in DMs, whereas, in the case of weak feedback scenario, the DM distribution is much broader. Therefore, we would get different DM distributions for FRBs at the same $z$ depending on how far the baryons are stretching the halos of the galaxies along the line of sight and location of baryons within the halo. We would also need to find redshift measurements for many FRBs to account for the effects of variance in the sightlines. Nonetheless, extragalactic DM measurements of FRBs do provide means of directly measuring the probability distribution of the intergalactic IGM and could constrain the locations of the cosmic baryons.

Recently, Yang \& Zhang (2016) showed that the average host galaxy DM and cosmological parameters including the matter density and baryonic density can be independently inferred from the slope of the first derivative of the $\mathrm{DM}_{\mathrm{E}}-z$ relation. $\mathrm{DM}_{\mathrm{E}}$ is the $\mathrm{DM}$ obtained after subtracting the Galactic contribution from the observed DM. This method requires a sample of FRBs for which DM and $z$ are 

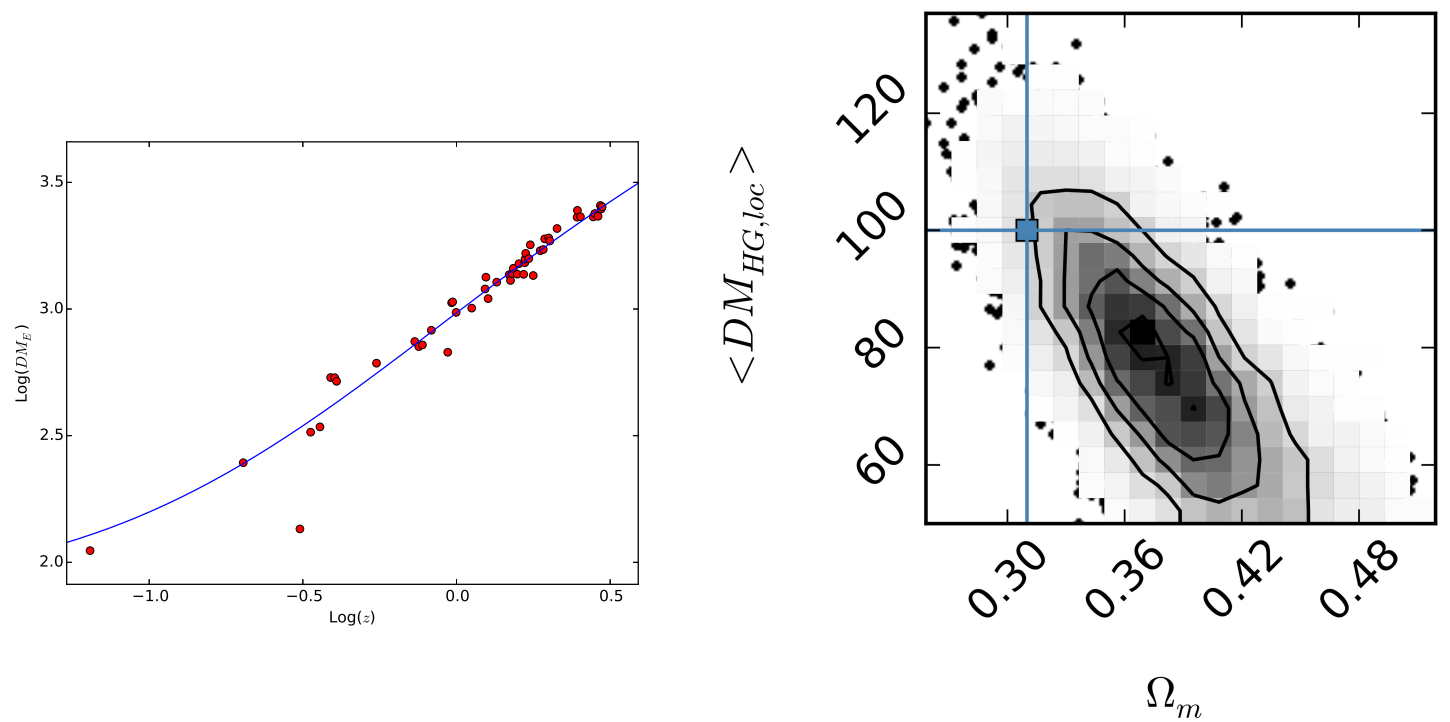

Figure 1.13: This figure is obtained from Yang \& Zhang (2016). The left panel shows the simulated FRBs (red dots) and their MCMC fitting curve (blue line). The right panel gives their estimates as $\mathrm{DM}_{\text {host }}=77.06 \mathrm{~cm}^{-3} \mathrm{pc}$ and $\Omega_{\mathrm{m}}=0.38$.

measured and a Markov Chain Monte Carlo (MCMC) fit can be applied to extract the $\mathrm{DM}_{\text {host }}$ and cosmological parameters using the slope $(\bar{\beta})$ of the $\mathrm{DM}_{\mathrm{E}}$ versus $z$ relation, and the mean values of $\mathrm{DM}_{\mathrm{E}}$ and $z$ of the sample. They define two quantities (see Equations 5 and 6 of Yang \& Zhang 2016) :

$$
\alpha(z) \equiv \frac{d \ln \left\langle\mathrm{DM}_{\mathrm{IGM}}\right\rangle}{d \ln z} \propto f\left(z, \Omega_{\mathrm{m}}, \Omega_{\Lambda}\right)
$$

And

$$
\bar{\beta}(z) \equiv \frac{d \ln \left\langle\mathrm{DM}_{\mathrm{E}}\right\rangle}{d \ln z} \propto g\left(z,\left\langle\mathrm{DM}_{\mathrm{E}}\right\rangle,\left\langle\mathrm{DM}_{\text {host }}\right\rangle\right) .
$$

At lower $z$, Equation 1.15 can give an estimate for $\mathrm{DM}_{\text {host }}$ using measured values of $\bar{\beta}$ and $z$. At higher redshifts, $\left\langle\mathrm{DM}_{\mathrm{E}}\right\rangle \gg\left\langle\mathrm{DM}_{\text {host }}\right\rangle$ and $\alpha(z) \simeq \bar{\beta}$, hence measuring $\bar{\beta}$ can give estimates for $\Omega_{\mathrm{m}}$. Their results are shown in Figure 1.13. In the left panel, the red dots denote the simulated FRBs and the blue line denotes their MCMC 
fitting curve. Since this method does not assume anything about the host galaxy DM, this method can place constraints on some of the progenitor models.

Previously, Zhou et al. (2014) demonstrated that if sufficient FRBs (few tens) are detected in a narrow redshift interval $(\Delta z \sim 0.05)$ then FRBs could help constrain the dimensionless parameter $w$ which characterizes the equation of state of dark energy with pressure $p$ and the dark energy density $\rho$

$$
w=\frac{p}{\rho} .
$$

They assume events with host galaxy DM less than $100 \mathrm{~cm}^{-3}$ pc. For a sample of 1000 FRBs with known $z$, DM can be estimated. This is shown in the inset of Figure 1.14. They performed a $\chi^{2}$ statistic and compared the data from SNe Ia (solid yellow lines), baryon acoustic oscillations data obtained from Sloan Digital Sky Survey shown as dotted blue lines, and FRB data as dashed red lines to estimate $w$.

Bonetti et al. (2016) analyzed the time delay between different frequencies for FRBs whose redshifts are measured and placed upper limits on the rest mass of the photon. Bonetti et al. (2017) estimated the rest mass to be $m_{\gamma}<1.77 \times 10^{44} \mathrm{~kg}$ for FRB 121102 at $z=0.193$. Overall, FRBs can help in answering some of the most fundamental questions in astrophysics. 


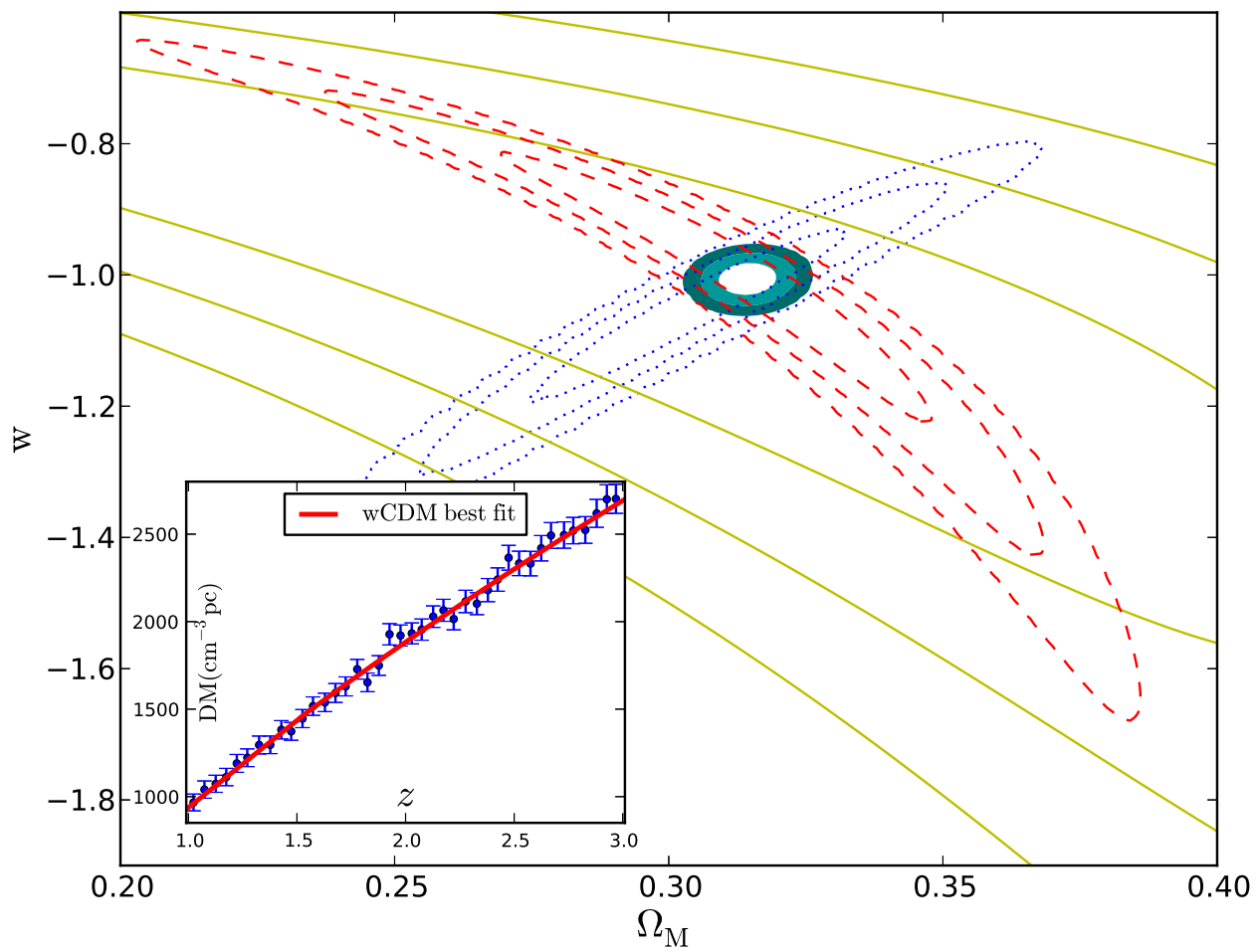

Figure 1.14: This figure is obtained from Zhou et al. (2014) showing the best-fit estimate for $w$ for 1000 simulated FRBs. 


\subsection{Dissertation Outline}

The outline of this dissertation is as follows. In Chapter 2, we describe the single-pulse search analysis and present the results of the search for dispersed radio bursts in the Parkes high-latitude pulsar survey. In Chapter 3, we discuss a Bayesian technique used to constrain the event rate of fast radio bursts using previous pulsar surveys carried out with the Parkes telescope. In Chapter 4, we present the search for host galaxies of potentially extragalactic rotating radio transients. In Chapter 5, we investigate the empirical population models for fast radio bursts. Finally, in Chapter 6, we present the main conclusions from this work and plans for future work. 


\section{Chapter 2}

A search for rotating radio transients and fast radio bursts in the

Parkes high-latitude pulsar survey

The work in this chapter was originally published as: A. Rane, D. R. Lorimer, S. D. Bates, N. McMann, M. A. McLaughlin, K. Rajwade, 'A search for rotating radio transients and fast radio bursts in the Parkes high-latitude pulsar survey', MNRAS, volume 455, p. 2207$2215,2016$.

\subsection{Introduction}

Although FRBs have so far been observed over a range of Galactic latitudes, their true distribution on the sky remains unclear. Based on an analysis of Parkes HTRU mid-latitude survey data, Petroff et al. (2014) proposed that there is a deficit of FRBs at intermediate latitudes. In this Chapter, we investigate this issue by analyzing results from archival and current surveys. In addition, Keane \& Petroff (2015) have assessed the commonly used search algorithms used for FRB searches which impact the FRB sensitivities in individual surveys. Motivated by these results, in this Chapter we present a single-pulse search which is sensitive to both RRATs (McLaughlin et al., 2006) and FRBs on archival data previously searched for pulsars at high Galactic latitudes by Burgay et al. (2006). 


\subsection{Survey parameters}

The Parkes high-latitude (PH) survey (Burgay et al., 2006) was designed to find millisecond pulsars and exotic binaries which migrate away from the Galactic plane. A total of 42 pulsars were detected in this survey, of which seven were millisecond pulsars and 18 were new discoveries. The analysis of the data by Burgay et al. (2006) was carried out using the standard periodicity search methods to find periodic signals. However no single-pulse searches have been published on these data. The single-pulse search method is very effective in detecting sporadic sources like some pulsars (e.g., nulling pulsars) and RRATs in the time domain since these might not be detectable in the standard periodicity searches (Cordes \& McLaughlin, 2003). Moreover, FRBs can of course only be detected through single-pulse searches (see below $\S 2.2 .2$ ).

The PH survey covered a strip of the sky enclosed by Galactic longitudes $220 \leq l \leq 260$ and Galactic latitudes $|b| \leq 60$ corresponding to a total sky coverage of 3588 deg $^{2}$ in 475 hours. The survey began in November 2000 and ended in December 2003 and made use of the 13-beam receiver on the Parkes 64-m radio telescope. Data were collected simultaneously by 13 beams at a central frequency of $1374 \mathrm{MHz}$ with 96 frequency channels, each $3 \mathrm{MHz}$ wide. Each of the 6456 pointings was observed for 265 s. For more details about the receiver system and data acquisition, see Burgay et al. (2006). 


\subsection{Single-pulse search method}

Each of these beams from the survey was processed independently using the SIGPROC software package ${ }^{1}$. The steps included in our analysis are:

1. dedisperse the raw data file at a range of trial DM values and remove radio frequency interference (RFI) at zero DM;

2. search for individual pulses in the time series above signal-to-noise $(\mathrm{S} / \mathrm{N})$ of five and with different widths;

3. apply the detection criteria to filter in terms of $\mathrm{DM}, \mathrm{S} / \mathrm{N}$, and number of beams;

4. manually inspect the resulting diagnostic plots.

We describe each step in detail below, and give the appropriate SIGPROC modules used.

\subsubsection{Dedispersion}

Radio signals are affected by interstellar dispersion, and as a result, the higher frequencies of the signal traveling faster through the interstellar medium arrive earlier than their lower frequency counterparts. The time delay between the two frequencies $\nu_{1}$ and $\nu_{2}$ is given by Equation 1.7. The dispersion measure allows us to distinguish between astrophysical and terrestrial signals. It causes a quadratic sweep across the band and may be removed by appropriately shifting the frequency

\footnotetext{
${ }^{1}$ http://sigproc.sourceforge.net
} 
channels. Each time series was dedispersed over a range of trial DM values using the dedisperse routine in SIGPROC. For this analysis, we have searched DMs in a range from $0-10^{4} \mathrm{~cm}^{-3} \mathrm{pc}$. The wide range of DM makes the search sensitive to events that are highly dispersed. The trial DM step sizes used in the analysis were calculated using an algorithm, dedisperse_all, originally described by Levin (2012) which accounts for the amount of pulse broadening caused by the size of the previous DM step and then determines the next trial DM. A total pulse width smearing due to the DM step in comparison to the value at the last DM is chosen to be $25 \%$ (see, $\S$ Appendix A). The total number of DMs searched was 249, as chosen optimally by this program. For the DM steps used in our analysis, the average S/N loss is $\sim 1.5 \%$ for DMs $<2000 \mathrm{~cm}^{-3}$ pc and for DMs between $2000-10000 \mathrm{~cm}^{-3}$ pc, the average $\mathrm{S} / \mathrm{N}$ loss is $\sim 2.5 \%$, calculated using Equations 12 and 13 of Cordes \& McLaughlin (2003). The dedisperse routine uses Equation 1 to calculate the time delays for each test DM and applies to frequency channels and the samples from each channel are then averaged to form a dedispersed time series. In addition to this, it also performs zero-DM subtraction (Eatough et al., 2009) on the time series to remove any RFI at zero DM.

\subsubsection{Single-pulse search}

Each dedispersed time series corresponding to a particular trial DM was searched for transient events of different widths via the matched filtering technique for top-hat pulses implemented in the program seek. This simple algorithm, which is an imple- 
mentation of the method described in Cordes \& McLaughlin (2003), saves individual events that deviate by five standard deviations from the mean of the time series. A number of adjacent samples are added to search for pulses of different widths. Each time series was smoothed 15 times, corresponding to a maximum smoothing of $2^{15}$ times the sampling interval, i.e., pulse widths out to $4.096 \mathrm{~s}$. If a pulse is detected in more than one of the smoothed times series, only the highest $\mathrm{S} / \mathrm{N}$ value is recorded. Following Burke-Spolaor et al. (2011), the number of independent trials

$$
N=N_{\mathrm{DM}} \sum_{j=0}^{\mathrm{j}_{\max }} \frac{N_{\mathrm{samp}}}{2^{j}},
$$

where $N_{\mathrm{DM}}$ is the number of DM trials, $N_{\text {samp }}$ is the number of samples in each dedispersed time series, and $j_{\max }$ corresponds to number of matched-filter widths used. We find $N=1.1 \times 10^{9}$ for our observation. For more details of this search method, see Cordes \& McLaughlin (2003).

Keane \& Petroff (2015) have assessed the performance of the search algorithms commonly being used to discover FRBs. The authors point out some important concerns where sensitivity to FRBs is often unnecessarily reduced and that the single-pulse search routines within the dedisperse_all and seek packages were less efficient compared to Heimdall and destroy packages (see, e.g., Petroff et al., 2014). Based on the simulations which used an injected signal of known strength, Keane \& Petroff (2015) demonstrated that the recovered S/N for dedisperse_all and seek was a function of pulse phase. We apply these results into our analysis, as described in $\S 4.3$. 


\subsubsection{Detection criteria}

These dispersed pulses can be displayed graphically using the plotpulses program and the resulting single-pulse plot can be seen in the left panel of Figure 2.1. In the DM histogram (top center of left panel), we are able to detect sources that emit many weak pulses. Such sources might not be detectable in the bottom plot of left panel. Conversely, the sources that emit only a few strong pulses may only be detectable in the bottom plot. Often a peak in the DM histogram at low DMs is seen which is indicative of RFI. In some cases, RFI is seen at all DMs for a certain time range which can further limit our ability to detect a transient event. This would happen if there is a strong source of RFI that causes the receiver to saturate or some other local interference that is so strong that it shows up at all DMs for that time range. The single-pulse plots thus obtained were inspected manually (e.g., by searching for a well-defined peak in the S/N versus DM plot, see Fig. 2.1). For manual inspection, we restricted the $\mathrm{S} / \mathrm{N}$ threshold to six in order to keep the number of potential candidates at a manageable level.

The event detected by Lorimer et al. (2007) was detected simultaneously in three beams and all of the other FRBs were detected in only a single beam. A strong signal appearing in all 13 beams simultaneously showing a dispersive delay is considered to have a terrestrial or instrumental origin (called perytons, BurkeSpolaor et al. 2011). The origin of these events has been recently identified as coming from a microwave oven when its door is opened prematurely and if the telescope is at an appropriate relative angle at that time (Petroff et al., 2015b). We 


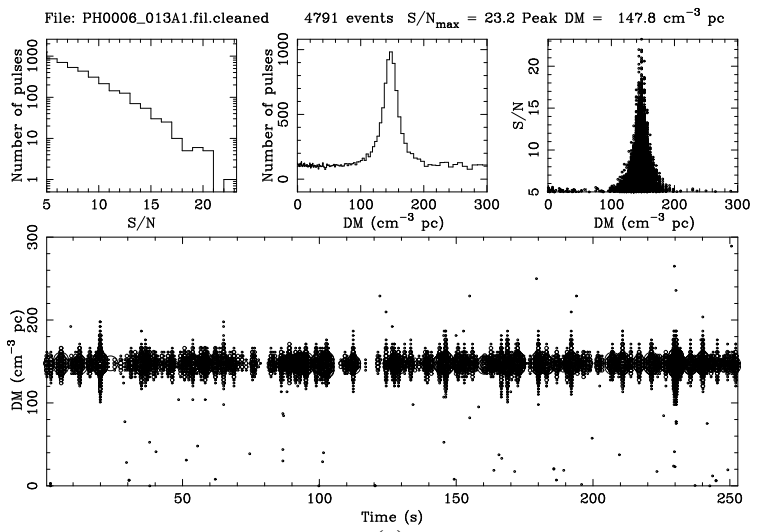

(a)

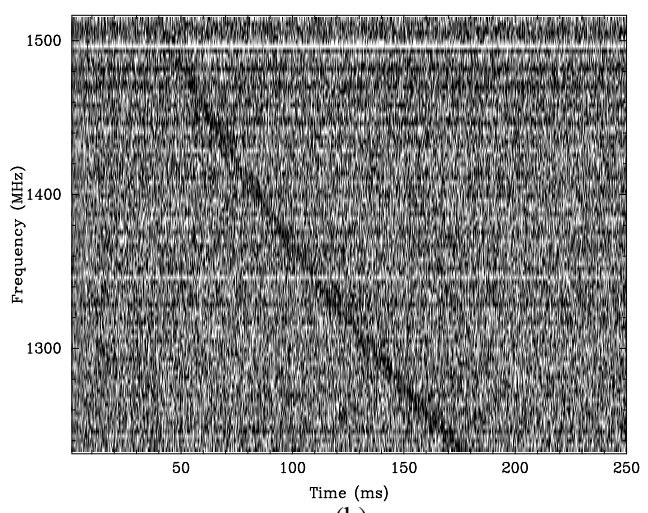

(b)

Figure 2.1: Example output of one single-pulse search processing pipeline, showing bright single pulses from PSR J0837-4135 around a DM value of $147 \mathrm{~cm}^{-3} \mathrm{pc}$. The top left panel shows the $\mathrm{S} / \mathrm{N}$ distribution of the detected pulses, number of pulses versus trial DM (in top center), and $\mathrm{S} / \mathrm{N}$ as a function of trial DM. The lower plot shows $\mathrm{S} / \mathrm{N}$ of events as a function of time and trial DM. The size of the circles is linearly proportional to the $\mathrm{S} / \mathrm{N}$ of each pulse. The dispersive delay in the frequency versus time plot is seen on the right for 250 milliseconds of data.

did not detect any such events in our analysis. We found a number of bursts with high $\mathrm{S} / \mathrm{N}$ in all 13 beams but no dispersive delay in the frequency versus time plot. These events are sources of RFI which have near earth origin and are only active for a brief period of time. Nearly $10 \%$ of the data show S/N greater than six in more than five beams but do not show dispersive delay. We did not consider these candidates further in our analysis.

\subsubsection{Manual inspection}

The diagnostic plots obtained after applying all the detection criteria were manually examined to look for a strong signal corresponding to a peak in the S/N versus DM plot. Such candidates are shortlisted and the detection is confirmed if the signal shows a sweep from high to low frequency across the observing bandwidth in 

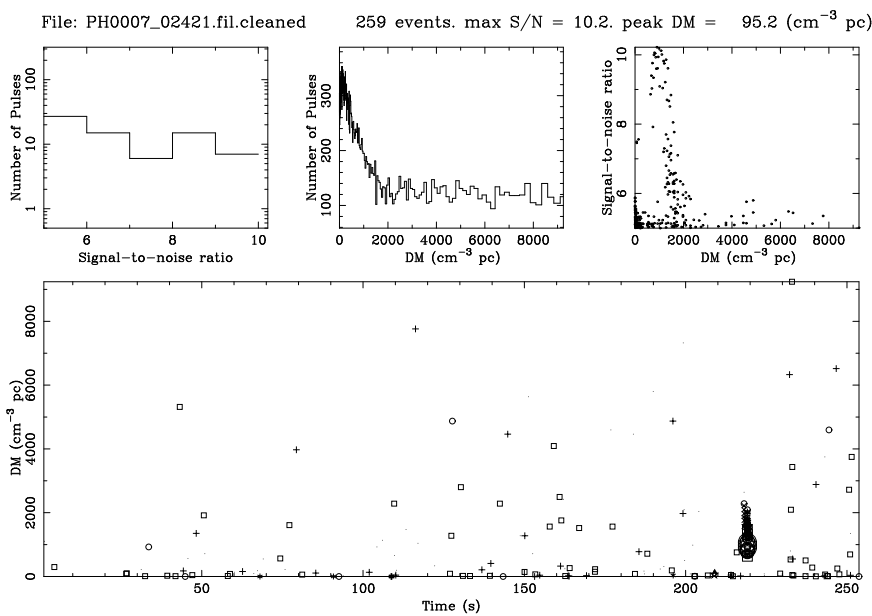

(a)

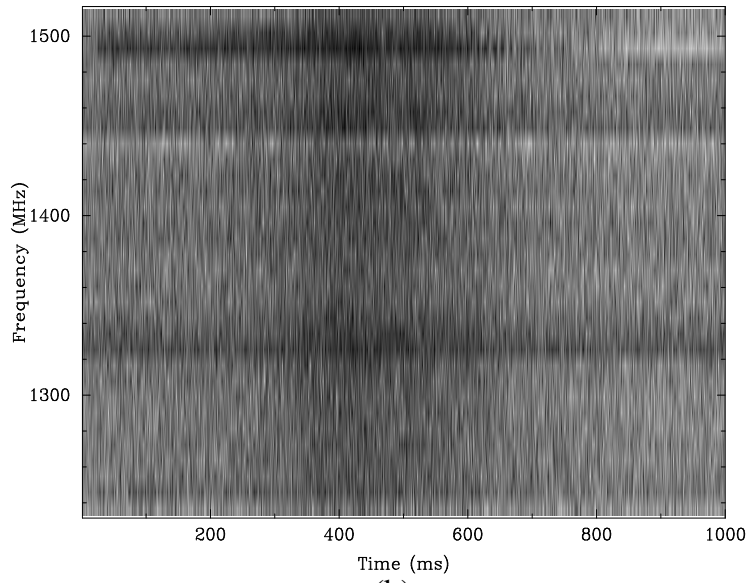

(b)

Figure 2.2: The left panel shows a non-astrophysical dispersed burst as seen in the lower plot and a corresponding peak in the $\mathrm{S} / \mathrm{N}$ versus $\mathrm{DM}$ plot. The nondedispersed data is then plotted corresponding to time of the peak for one second as seen in the right panel here, confirming that it is a false detection.

the frequency versus time plot. Brightness was not a criterion for being shortlisted and some candidates were confirmed as pulsars despite not being detectable in this plot. For FRBs, if a dispersive sweep is absent then it is deemed to be a "false detection" (see, e.g., Figure 2.2). A burst-like event was seen in one of the beams (see a bright pulse in the lower plot of Figure 2.2 and a corresponding peak in the $\mathrm{S} / \mathrm{N}$ versus DM plot in the upper right plot) but no dispersed signal was detected in the frequency versus time plot (see right panel of Figure 2.2).

\subsection{Results}

As summarized in Table 2.4, our single-pulse search resulted in the detection of 20 of the 42 pulsars detected in the original periodicity search by Burgay et al. (2006). In addition, one RRAT not reported by Burgay et al. (2006) was detected. The discovery of this source, PSR J0410-3113, was reported by Burke-Spolaor et 
al. (2011) during a single-pulse search on the data obtained from the high-latitude HTRU survey. In our analysis, only one pulse was detected in this observation for PSR J0410-3113, consistent with the non-detection in the periodicity search ${ }^{2}$.

\subsection{Energy measurements}

Integrated profiles for each pulsar were obtained by folding the dedispersed time series using ephemerides available from the ATNF pulsar catalog (Manchester et al., 2005). To construct the pulse energy histograms, the procedure described in Ritchings (1976) is followed. The position and widths of the on-pulse and offpulse windows were determined by visual inspection of the integrated pulsar profile. Baseline estimation was done using the off-pulse bins, and was subtracted from the data for each pulse. The total energy for each pulse in the on-pulse window was calculated and is scaled to account for different widths of on and off windows. The on-pulse data were taken in blocks of about 100 pulses. The on-pulse and off-pulse normalized energies $E / \bar{E}$ were calculated for each block by dividing on-pulse and off-pulse energies within the block by the mean on-pulse energy of that block to account for variations due to interstellar scintillation. The pulse energy histograms were constructed for 10 of the total 17 re-detected pulsars, as shown in Fig. 2.3, based on the number of pulses detected during the total observation time. We did not create energy distribution plots for sources with fewer than 100 pulses per observation.

\begin{tabular}{cccccc}
\hline${ }^{2}$ The ATNF pulsar catalog can online at \\
http://www.atnf.csiro.au/research/pulsar/psrcat
\end{tabular}


Table 2.1: All pulsars detected and discovered in the PH survey. Columns 1 to 5 report the pulsar name, Galactic longitude and latitude, spin period, and DM, all obtained from the ATNF pulsar catalog (Manchester et al., 2005). For those that were detected in our single-pulse search method, columns 6, 7, 8, and 9 report the $\mathrm{DM}$ obtained in this analysis, peak $\mathrm{S} / \mathrm{N}$ from single-pulse search, number of pulses, and width. Column 10 lists S/N from the periodicity search obtained from Burgay et al. (2006) and column 11 lists the intermittency measure. The RRAT discovered by Burke-Spolaor et al. (2011) and re-detected in our analysis is denoted by * .

\begin{tabular}{|c|c|c|c|c|c|c|c|c|c|c|}
\hline $\begin{array}{l}\text { Name } \\
\text { PSR }\end{array}$ & $\begin{array}{l}l \\
()\end{array}$ & $\begin{array}{l}b \\
()\end{array}$ & $\begin{array}{c}P \\
(\mathrm{~ms})\end{array}$ & $\begin{array}{c}D M \\
\left(\mathrm{pc} \mathrm{cm}^{-3}\right)\end{array}$ & $\begin{array}{c}D M_{\mathrm{obs}} \\
\left(\mathrm{pc} \mathrm{cm}^{-3}\right)\end{array}$ & $(S / N)_{\mathrm{SP}}$ & $N_{\text {pulses }}$ & $\begin{array}{c}W \\
(\mathrm{~ms})\end{array}$ & $(S / N)_{\text {per }}$ & $r$ \\
\hline J0343-3000 & 227.76 & -52.34 & 2597.02 & 20.2 & 22.3 & 21.5 & 22 & 2.0 & 42.7 & 0.50 \\
\hline J0410-3113* & 253.47 & -41.95 & 1837.00 & 9.9 & 9.9 & 9.2 & 1 & 4.0 & - & - \\
\hline J0437-4715 & 253.47 & -41.95 & 5.70 & 2.6 & 2.6 & 12.8 & 4303 & 0.3 & 603.3 & 0.02 \\
\hline J0448-2749 & 228.43 & -37.91 & 450.40 & 26.2 & 26.4 & 10.2 & 6 & 4.0 & 28.5 & 0.38 \\
\hline J0520-2553 & 228.51 & -30.53 & 241.60 & 33.7 & - & - & - & - & 34.0 & - \\
\hline J0610-2100 & 227.75 & -18.18 & 3.86 & 60.6 & _- & _- & _- & _- & 10.1 & _ \\
\hline J0630-2834 & 237.03 & -16.75 & 1244.40 & 34.5 & 35.3 & 20.2 & 63 & 16.0 & 277.4 & 0.07 \\
\hline J0633-2015 & 229.33 & -12.95 & 3253.21 & 90.7 & 89.7 & 11.0 & 3 & 8.0 & 16.4 & 0.67 \\
\hline J0636-4549 & 254.55 & -21.55 & 1984.59 & 26.3 & - & - & - & - & 11.2 & - \\
\hline J0656-2228 & 233.66 & -8.98 & 1224.75 & 32.4 & 31.1 & 6.9 & 29 & 0.3 & 21.0 & 0.33 \\
\hline J0719-2545 & 238.93 & -5.83 & 974.72 & 253.9 & - & - & - & - & 20.7 & - \\
\hline J0726-2612 & 240.08 & -4.64 & 3442.31 & 69.4 & 68.8 & 16.8 & 9 & 4.0 & 15.1 & 1.11 \\
\hline J0729-1448 & 230.46 & 1.44 & 251.60 & 92.3 & - & - & - & - & 32.5 & - \\
\hline J0729-1836 & 233.83 & -0.33 & 510.10 & 61.2 & 61.0 & 7.4 & 26 & 0.3 & 59.3 & 0.13 \\
\hline J0737-3039A & 245.24 & -4.50 & 22.70 & 48.9 & - & - & - & - & 18.7 & - \\
\hline J0737-3039B & 245.24 & -4.50 & 2773.46 & 48.9 & - & - & - & - & - & - \\
\hline J0738-4042 & 254.27 & -9.1 & 374.90 & 160.8 & 161.3 & 20.8 & 644 & 2.0 & 542.1 & 0.04 \\
\hline J0742-2822 & 243.85 & -2.43 & 166.70 & 73.8 & 73.0 & 18.3 & 2130 & 2.0 & 63.1 & 0.29 \\
\hline J0746-4529 & 259.20 & -10.10 & 2791.03 & 134.6 & - & - & - & - & 10.3 & - \\
\hline J0749-4247 & 257.14 & -8.33 & 1095.40 & 104.5 & - & - & - & - & 17.6 & - \\
\hline J0758-1528 & 234.54 & 7.24 & 682.20 & 63.3 & 62.9 & 13.4 & 67 & 8.0 & 99.0 & 0.14 \\
\hline J0818-3232 & 251.36 & 1.87 & 2161.26 & 131.8 & - & - & - & - & 27.3 & - \\
\hline J0820-1350 & 235.96 & 12.61 & 1238.10 & 40.9 & 39.9 & 19.0 & 28 & 16.0 & 112.5 & 0.17 \\
\hline J0820-3921 & 257.26 & -1.58 & 1073.57 & 179.4 & - & - & - & - & 13.4 & - \\
\hline J0820-4114 & 258.82 & -2.72 & 545.40 & 113.4 & - & - & - & - & 42.6 & - \\
\hline J0821-4221 & 259.83 & -3.14 & 396.73 & 270.6 & - & - & - & - & 10.8 & - \\
\hline J0823+0159 & 222.06 & 21.26 & 864.80 & 23.7 & 22.3 & 15.8 & 54 & 8.0 & 13.1 & 1.21 \\
\hline J0828-3417 & 254.04 & 2.58 & 1848.90 & 52.2 & 52.5 & 13.3 & 5 & & 49.2 & 0.27 \\
\hline J0835-3707 & 257.15 & 2.00 & 541.40 & 112.3 & 113.5 & 6.9 & 1 & 8.0 & 33.2 & 0.21 \\
\hline J0837-4135 & 260.98 & -0.32 & 751.60 & 147.3 & 147.8 & 23.2 & 303 & 4.0 & 152.2 & 0.15 \\
\hline J0838-2621 & 248.81 & 8.98 & 308.58 & 116.9 & - & - & - & - & 9.6 & - \\
\hline J0843+0719 & 219.40 & 28.22 & 1365.86 & 36.6 & - & - & - & - & 15.9 & - \\
\hline J0846-3533 & 257.26 & 4.72 & 1116.00 & 94.1 & - & - & - & - & 103.1 & - \\
\hline J0855-3331 & 256.92 & 7.53 & 1267.50 & 86.6 & 89.7 & 9.9 & 12 & 8.0 & 42.5 & 0.23 \\
\hline J0900-3144 & 256.16 & 9.49 & 11.11 & 75.7 & - & - & - & - & 20.8 & - \\
\hline J0908-1739 & 246.19 & 19.86 & 401.60 & 15.8 & - & - & - & - & 23.9 & - \\
\hline J0922+0638 & 225.48 & 36.40 & 430.60 & 27.3 & 26.4 & 18.2 & 195 & 4.0 & 96.8 & 0.19 \\
\hline J0944-1354 & 249.20 & 28.86 & 570.20 & 12.4 & - & - & - & - & 43.1 & - \\
\hline J0953+0755 & 228.97 & 43.71 & 253.00 & 2.9 & 3.0 & 14.0 & 657 & 2.0 & 128.7 & 0.11 \\
\hline $\mathrm{J} 1022+1001$ & 231.86 & 51.11 & 16.40 & 10.3 & 10.4 & 9.6 & 266 & 0.5 & 318.7 & 0.03 \\
\hline J1024-0719 & 251.77 & 40.53 & 5.10 & 6.4 & - & - & - & - & 33.4 & - \\
\hline $\mathrm{J} 1038+0032$ & 247.15 & 48.47 & 28.85 & 26.6 & - & - & - & - & 18.1 & - \\
\hline $\mathrm{J} 1046+0304$ & 246.48 & 51.71 & 326.20 & 25.3 & 28.2 & 9.8 & 1 & 8.0 & 25.5 & 0.38 \\
\hline
\end{tabular}


For some pulsars, the on-pulse distribution peaks at the mean energy, as seen for PSR J0742-2822, PSR J0630-2834, PSR J0837-4135, and PSR J0922+0638. In the case of PSR J0742-2822, the energy histograms separate out clearly with no zero energy excess in the ON histogram, suggesting that this pulsar does not null. For other pulsars (PSR J0448-2749, PSR J0828-3417, and PSR J1046+0304), the histograms overlap just because the $\mathrm{S} / \mathrm{N}$ is low and not necessarily because the pulsar actually nulls. But it is important to note here that the statistics are limited by the relatively short observation time in the survey. So, we did not fit any Gaussians to the histograms since the errors would be large. Even if the pulsar is nulling, there are insufficient pulses to form a distribution for estimation of the nulling fraction in our analysis.

\subsection{Intermittency measure}

The two search algorithms used for pulsar searching show varying levels of efficiency which depend upon the properties of each particular pulsar (McLaughlin \& Cordes, 2003). Following Deneva et al. (2009), we calculated the intermittency ratio

$$
r=\frac{(S / N)_{\mathrm{SP}}}{(S / N)_{\mathrm{per}}}
$$

for each pulsar from the $\mathrm{S} / \mathrm{N}$ value of the single-pulse and periodicity searches and $r$ is plotted versus the number of periods in $\mathrm{T}_{\mathrm{obs}}$ (see Fig. 2.4). All the pulsars re-detected in our analysis except PSR J0410-3113 were detected in a previous periodicity search. The pulsars on the upper left have longer periods and the pul- 


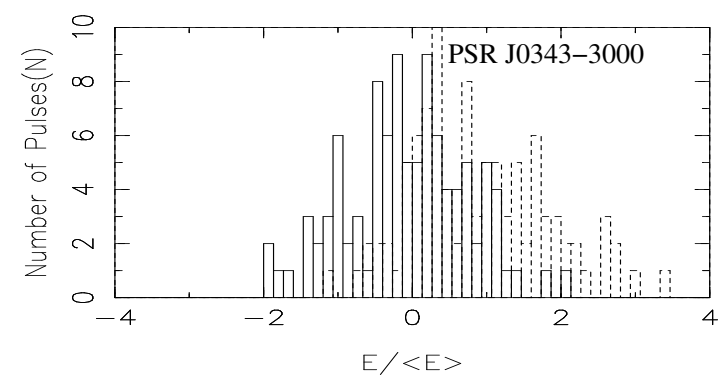

(a)

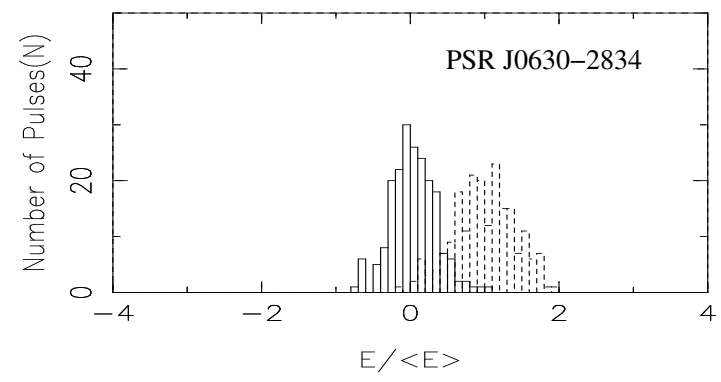

(c)

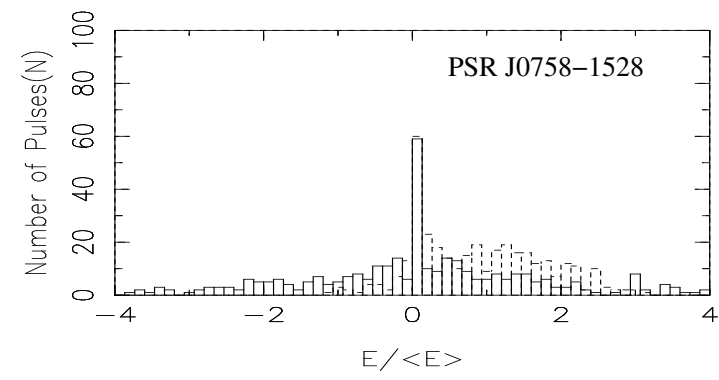

(e)

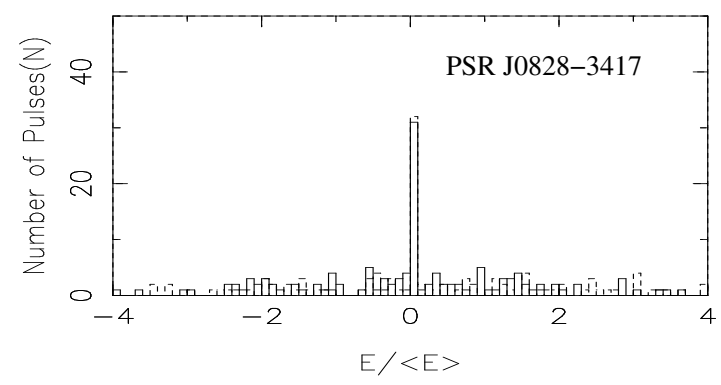

(g)

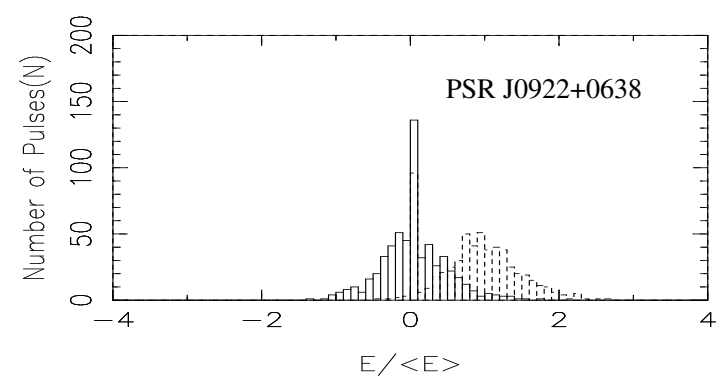

(i)

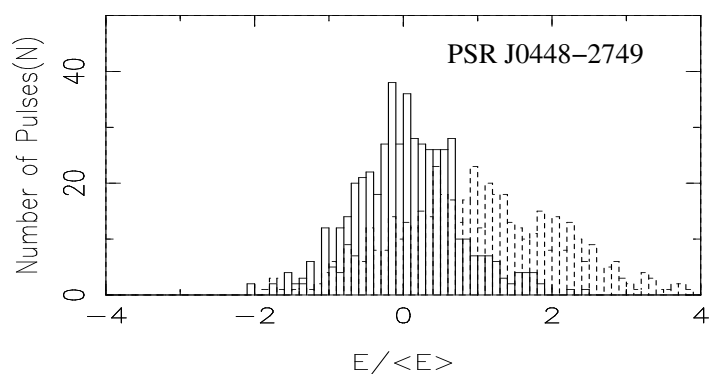

(b)

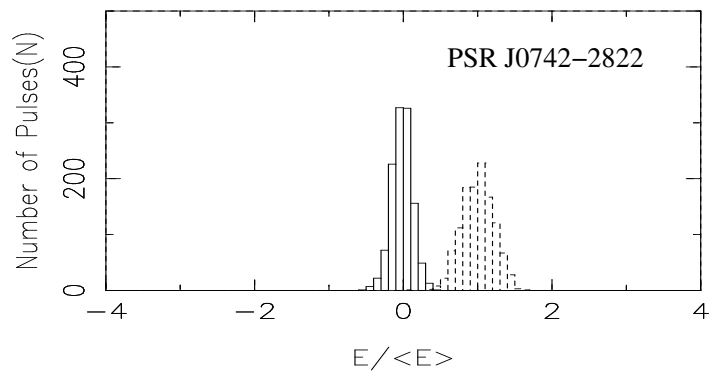

(d)

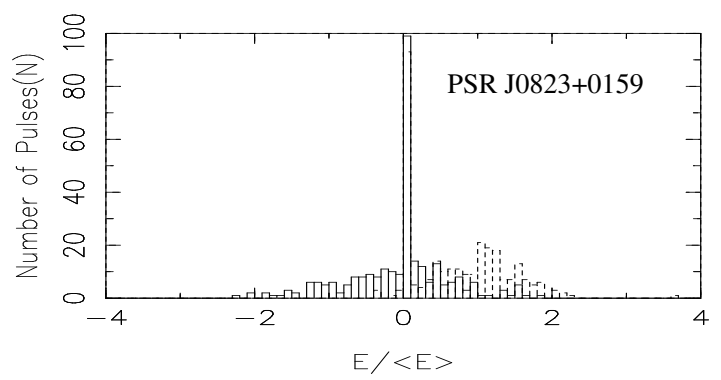

(f)

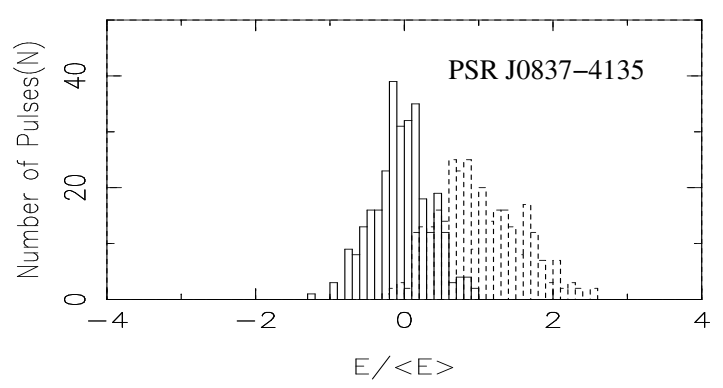

(h)

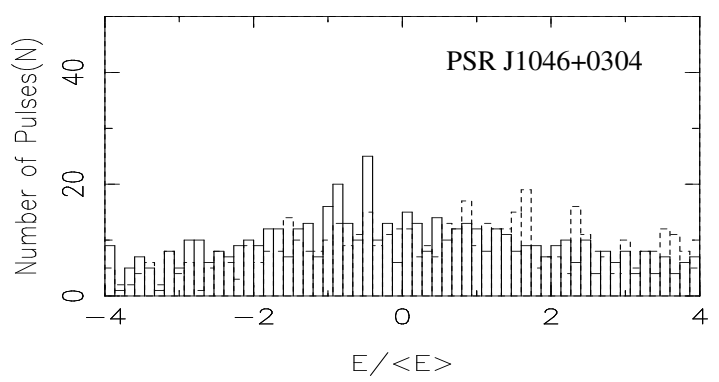

(j)

Figure 2.3: Normalized histograms of on-pulse (dashed) and off-pulse (solid) energies, for 10 of the total 21 re-detected pulsars. 


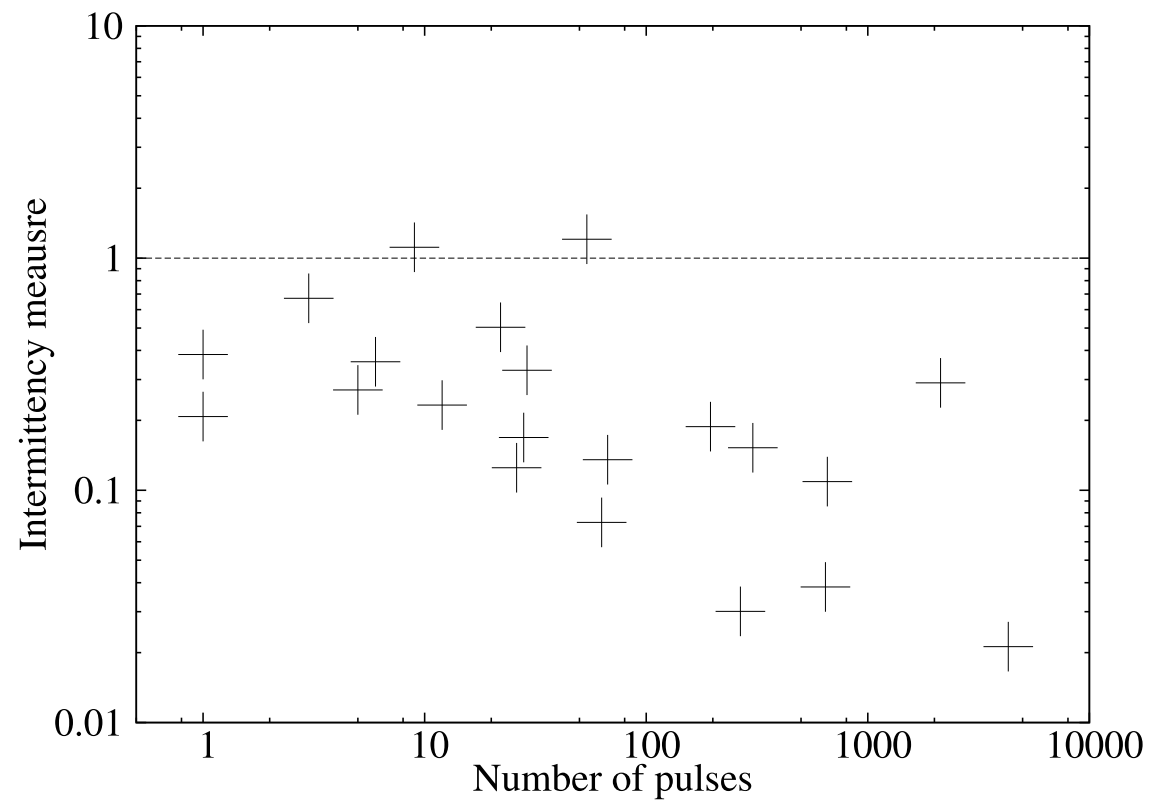

Figure 2.4: Intermittency measure for each pulsar detection in our survey.

sars on the lower right of this plot are millisecond pulsars. Pulsars with $r>1$ (PSRs J0726-2612 and J0823+0159) are more likely to be detected with single-pulse searches. PSR J0726-2612, with $r \sim 1.1$, has $P=3.4 \mathrm{~s}$ and DM $=69 \mathrm{pc} \mathrm{cm}^{-3}$ and PSR J0437-4715, with $r \sim 0.02$, has $P=5.7 \mathrm{~ms}$ and $\mathrm{DM}=2.6 \mathrm{pc} \mathrm{cm}^{-3}$. These ratios are higher than one would expect in longer surveys, because of the dependence of sensitivity on number of pulses. The integration time for this survey was relatively short (265 s) and we detected $50 \%$ of pulsars from single-pulse search. This can be compared to the PM survey with long integration time (2100 s), in which the single-pulse detections were only about $30 \%$ of the total pulsar discoveries from periodicity search (Keane et al., 2010). 


\subsection{Conclusions}

We have presented the results of a single-pulse search of the PH pulsar survey. We re-detected 20 of the previously known pulsars reported by Burgay et al. (2006) and detected one RRAT. No FRBs were found in this analysis. Out of these 21 re-detections, we have constructed pulse energy histograms for 10 pulsars for which the observed number of pulses was more than 100 . For PSR J0742-2822, the onpulse and off-pulse histograms separate out clearly, suggesting it does not null over the observing span. The observation time, being relatively short, is insufficient for estimation of the nulling fraction. 


\section{Chapter 3}

\section{Event rate of Fast Radio Bursts}

The work in $\S 3.1, \S 3.2$, and $\S 3.3$ of this chapter was originally

published as: A. Rane, D. R. Lorimer, S. D. Bates, N. McMann, M. A. McLaughlin, $K$. Rajwade, 'A search for rotating radio transients and fast radio bursts in the Parkes high-latitude pulsar survey', MNRAS, volume 455, p. 2207-2215, 2016. The work in $\S 3.4$ of this chapter was originally published as: F. Crawford, A. Rane, L. Tran, K. Rolph, D. R. Lorimer, J. P. Ridley, 'A search for highly dispersed fast radio bursts in three Parkes multibeam surveys', MNRAS, volume 460, p. 3370-3375, 2016.

\subsection{Introduction}

In this Chapter, we further investigate the results presented in Chapter 2. Although our analysis did not result in any new FRB detections in the Parkes high latitude survey, it is important to consider the impact of this null result on the FRB event rate $\mathcal{R}$. To put our results into context, we also consider a number of other surveys at Parkes in the calculation presented in this Chapter. 


\subsection{Bayesian Approach}

To constrain $\mathcal{R}$, we apply a Bayesian approach which uses the FRB detections in each survey. Using Bayes' theorem (see, e.g., Wall \& Jenkins 2003), the posterior probability density function for $\mathcal{R}$, given the detection of $n$ pulses

$$
p(\mathcal{R} \mid n) \propto p(n \mid \mathcal{R}) p(\mathcal{R})
$$

where $p(n \mid \mathcal{R})$ is the likelihood of getting $n$ detections given some $\mathcal{R}$ and $p(\mathcal{R})$ is the prior on the rate of FRBs considered to be a flat prior that assigns equal likelihood on all possible values of the parameter. Taking $n=0$ from Petroff et al. (2014) and assuming the counting of these rare events as a Poisson process, the likelihood function

$$
p(0 \mid \mathcal{R})=\frac{\left(\mathcal{R} A_{P} T_{P}\right)^{0} \exp \left(-f \mathcal{R} T_{P} A_{P}\right)}{0 !}
$$

where $A_{\mathrm{P}}=4449 \mathrm{deg}^{2}$ and $T_{\mathrm{P}}=540 \mathrm{sec}$ is the total observation time for each pointing. The subscript $P$ corresponds to the values for Parkes. The numerical factor $f=(86400 \times 41253)^{-1}$ is inserted to compute $\mathcal{R}$ in units of bursts day ${ }^{-1}$ sky $^{-1}$. The posterior probability density of the FRB event rate can be computed as

$$
p(\mathcal{R} \mid 0)=K_{1} \mathcal{R}^{0} \exp \left(-f \mathcal{R} T_{P} A_{P}\right),
$$

where $K_{1}$ is a normalizing constant which ensures that the above expression integrates over all values of $\mathcal{R}$ to unity. Integrating this function, we find that 
the mean FRB rate based on zero FRBs in the HTRU mid-latitude survey is $\mathcal{R}=0.22_{-0.21}^{+4.5} \times 10^{3} \mathrm{FRBs}_{\text {day }}{ }^{-1} \mathrm{sky}^{-1}$, where the uncertainties represent the $99 \%$ confidence interval. To include the FRB detections and null results from subsequent surveys, a similar calculation is performed to determine the FRB event rates for individual surveys as listed in Table 3.1.

The surveys were processed using different search algorithms and therefore the rates need to be modified following the results of Keane \& Petroff (2015) in order to combine the individual event rates. As per the response curve shown in Figure 1 of Keane \& Petroff (2015), we get the corrected S/N from the use of these algorithms by defining the efficiency factors, $\eta_{\text {ddall }}$ and $\eta_{\text {seek }}$. For dedisperse_all, we find

$$
\eta_{\text {ddall }}=\frac{S / N_{\text {dedisperse_all }}}{S / N_{\max }}=0.83
$$

and, for seek,

$$
\eta_{\text {seek }}=\frac{S / N_{\text {seek }}}{S / N_{\max }}=0.89 .
$$

\subsection{Discussion}

The FRB searches we consider in this analysis are the results of reprocessing the Parkes Multibeam (PMPS) survey which used destroy (Keane et al., 2010, 2011), the PH survey which used seek (this paper), the Swinburne intermediate and high-latitude (SWIN) surveys which used dedisperse_all (Edwards et al., 2001; Jacoby et al., 2009; Burke-Spolaor \& Bannister, 2014), the HTRU high-latitude (HTRU high) survey which used dedisperse_all (Thornton et al., 2013; Thorn- 
ton, 2013), and the HTRU mid-latitude (HTRU mid) survey which used Heimdall (Petroff et al., 2015a). In addition to this, the HTRU surveys were carried out using the digital back-end, Berkeley-Princeton-Swinburne Recorder (BPSR), whereas the older Parkes surveys used the analogue filterbank (AFB). In order to compare all Parkes surveys, the digitization loss factors $\beta$ depending on the back-end used need to be considered in our analysis. For AFB, $\beta=1.25$ and for BPSR, $\beta=1.07$ (Kouwenhoven \& Voûte, 2001). We then insert the efficiency factors into the minimum fluence equation calculated for each processed survey

$$
F_{\text {min }}=\frac{\beta T_{\text {sys }}(S / N)_{\min }}{G \eta_{\text {soft }} \sqrt{N_{\mathrm{p}} \Delta \nu}} \sqrt{W}
$$

where $T_{\text {sys }}=28 \mathrm{~K}$ is the system temperature, $G=0.66 \mathrm{~K} / \mathrm{Jy}$ is the telescope gain, $N_{\mathrm{p}}$ is number of polarizations, $\Delta \nu$ is the bandwidth, $\eta_{\text {soft }}$ is either $\eta_{\text {seek }}$ or $\eta_{\text {ddall }}$ depending the survey, and $w$ is the pulse width equal to the sampling time when calculating the minimum fluence. The HTRU mid-latitude survey has the lowest minimum fluence as can be seen in Table 3.1. The event rates of other surveys are scaled to this lowest $F_{\min }^{-3 / 2}$ which is the simplest model assuming a uniform distribution of standard-candle FRBs in Euclidean geometry. The modified event rates are shown in Fig. 3.1. Combining all these individual rate estimates, Fig. 3.2 shows our current best estimate of $\mathcal{R}$. The mean FRB rate from this distribution is $4.4_{-3.1}^{+5.2} \times 10^{3} \mathrm{FRBs} \mathrm{day}^{-1} \mathrm{sky}^{-1}$ for sources with a fluence above $4.0 \mathrm{Jy} \mathrm{ms}$ at 1.4 GHz, where the uncertainties represent a $99 \%$ confidence interval. 


\subsection{All-sky event rate of FRBs}

To demonstrate that this rate is consistent with all the surveys considered here, we list in Table 3.1 the predicted upper and lower bounds on the number of FRBs expected in each survey which use these $99 \%$ confidence intervals on $\mathcal{R}$ and scale it back to each survey's fluence limit. In addition, we make predictions for future analyses of the Perseus Arm (PA) pulsar survey (Burgay et al., 2013) and HTRU low-latitude survey (Thornton, 2013). The event rate is estimated assuming FRBs are uniformly distributed on the sky. We now consider the impact of this assumption. The Galactic effects such as dispersion in the ISM, scattering in the ISM, scintillation, and sky temperature can limit the sensitivity of a survey. Following the discussion in Petroff et al. (2014) about decreased sensitivity to FRBs at $|b| \leq 15$, we compare the sensitivity based on sky coverage for the Parkes surveys considered in our calculations. The non-Galactic DM contribution at high latitudes range between 520 and $1070 \mathrm{~cm}^{-3}$ pc. The Galactic dispersion at these latitudes is

only about $50 \mathrm{~cm}^{-3} \mathrm{pc}$, whereas the average Galactic dispersion at intermediate latitudes and near the Galactic center are $380 \mathrm{~cm}^{-3} \mathrm{pc}$ and $1780 \mathrm{~cm}^{-3}$ pc respectively. The FRB pulses with an additional non-Galactic DM contribution at these lower latitudes would still be recovered in the surveys considered above as they have been searched to a sufficient DM, with the maximum trial DM in each of these surveys being $>2000 \mathrm{~cm}^{-3} \mathrm{pc}$.

The average sky temperature values for four of these surveys range between 0.85 K to 3.18 K and about 6.14 K for the PMPS survey (Burke-Spolaor \& Bannister, 
2014). Sky temperature is therefore not a significant factor in limiting the sensitivity when comparing these surveys.

FRBs discovered so far (except FRB 110220) show few effects of scattering (see, e.g., Lorimer et al., 2013). Petroff et al. (2014) determined that more than $85 \%$ of survey pointings in the intermediate latitude survey are still sensitive to FRB signals even in the presence of strong scattering in the ISM near the Galactic center. This percentage will differ for AFB surveys as the number of channels, sampling time, and digitization factors are different but is still small compared to overall uncertainty in the FRB event rate at this point.

Petroff et al. (2014) analyzed how the combination of these effects limits sensitivity for survey pointings and determined that a simulated FRB pulse with properties similar to the FRBs in Thornton (2013) falls below the detection threshold in only $14 \%$ of all intermediate latitude pointings assuming no scattering in the ISM. We combine the individual rates since the sensitivity variations between AFB and BPSR are within the uncertainty of rate of FRBs. Petroff et al. (2014) argue that since the percentage of pointings no longer sensitive to FRB pulses decreases to $14 \%$, the null result is discrepant with the original predictions based on a higher event rate. Although, if the event rate of FRBs is much lower than previous estimates, it still explains the null result at intermediate latitudes (Table. 3.1). Therefore, we argue that the lack of detections at intermediate latitudes and the null result in our analysis presented in Chapter 2 are likely to be due to a lower FRB rate and does not necessarily imply a dearth of FRBs at intermediate latitudes. 


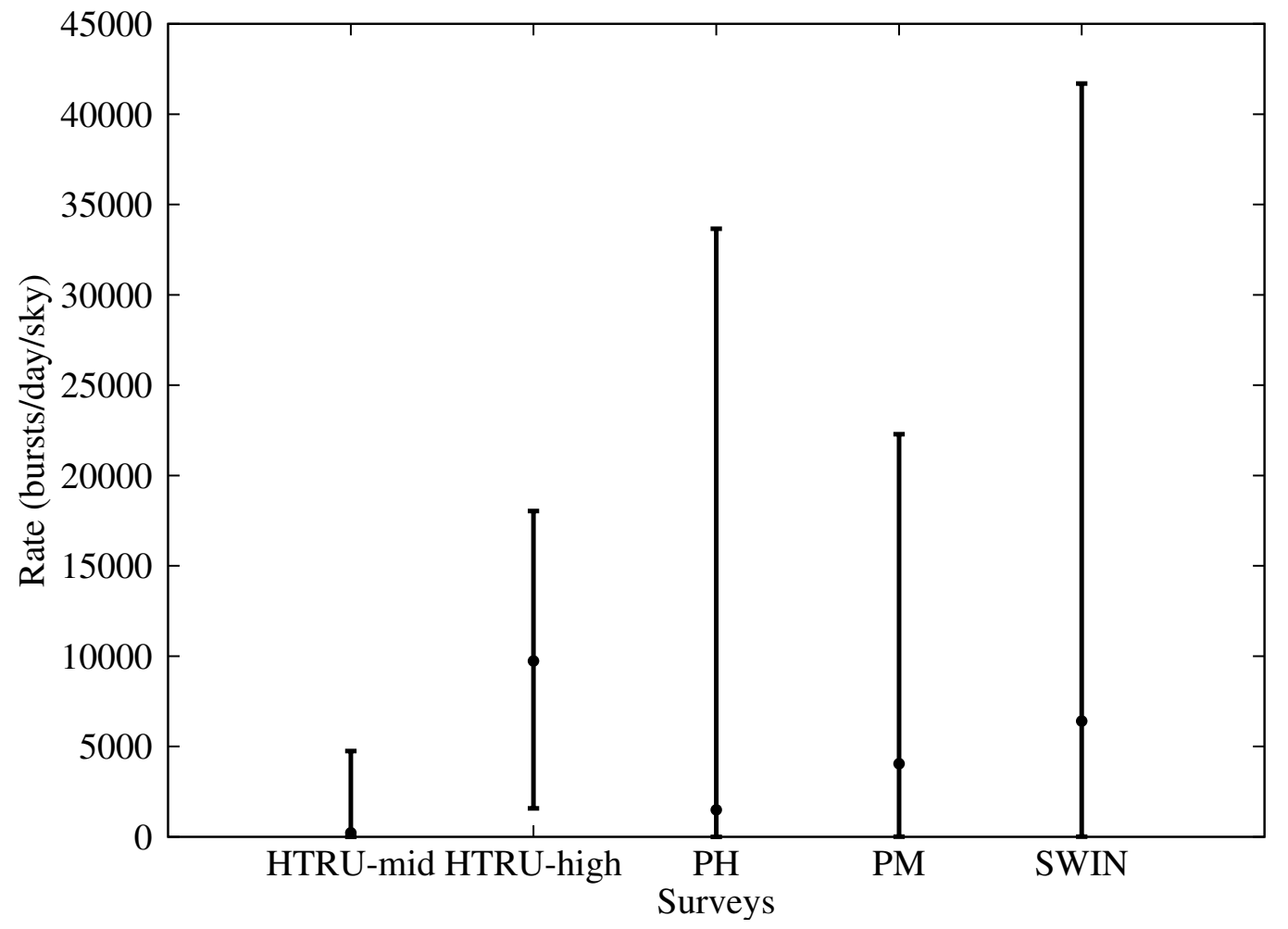

Figure 3.1: The modified FRB event rates based on individual survey results and corrected for detection sensitivity based on the search algorithms and the backend used. 


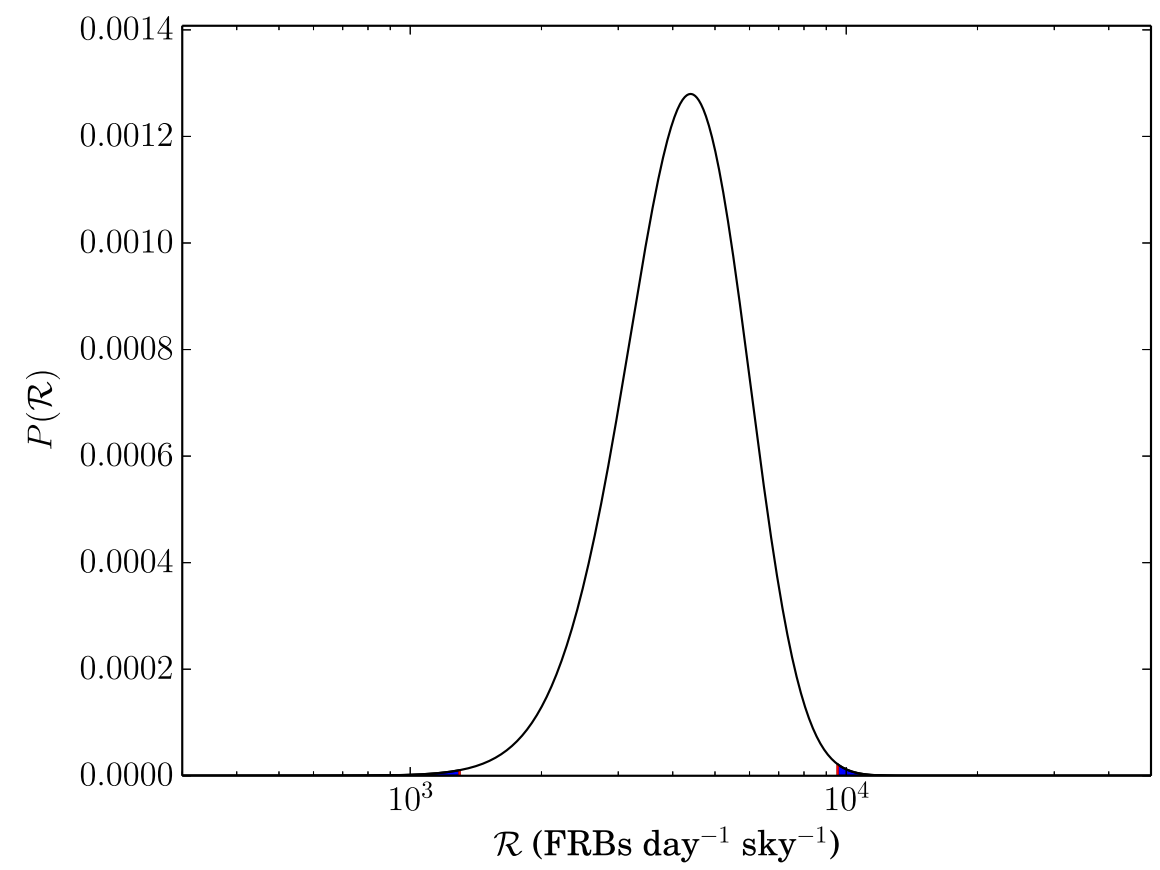

Figure 3.2: The combined posterior PDF of the FRB event rate taking into account all the published Parkes survey results carried out to date and correcting for detection sensitivity based on the search algorithms used and the backend used. The all-sky event rate for sources with a fluence above $4.0 \mathrm{Jy} \mathrm{ms}$ is $\mathcal{R}=4.4_{-3.1}^{+5.2} \times 10^{3} \mathrm{FRBs}_{\mathrm{day}}{ }^{-1} \mathrm{sky}^{-1}$. 


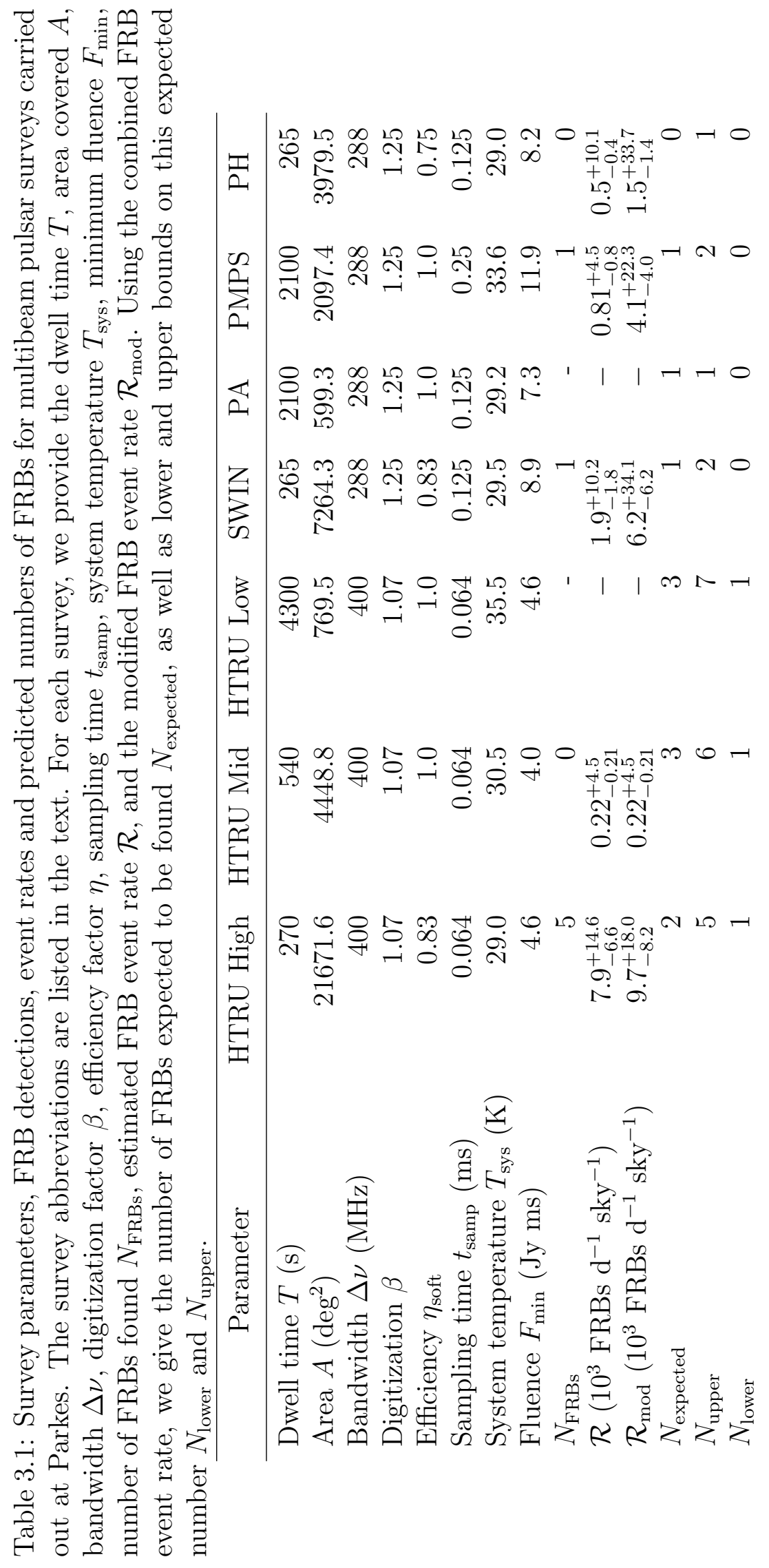




\subsection{Additional Parkes surveys and modified event rate}

In addition to the Parkes high latitude survey, Crawford et al. (2016) processed three more surveys to search for highly dispersed bursts. These surveys originally targeted the Magellanic Clouds (in two cases) and unidentified gamma-ray sources at mid-Galactic latitudes (in the third case) for new radio pulsars. The results obtained from the processing of these surveys are combined with results from previous surveys to determine a new constraint on the all-sky FRB rate. These other large-scale Parkes surveys have all been searched out to high DMs (at least a DM of 3000 pc $\mathrm{cm}^{-3}$; see Table 2 of Burke-Spolaor \& Bannister 2014).

To summarize:

- The HTRU intermediate-latitude survey (Petroff et al., 2014). A total of 1154 hr of on-sky time was recorded. No new FRBs were found.

- The Swinburne Multibeam (SWIN) survey (Burke-Spolaor \& Bannister, 2014). $925 \mathrm{hr}$ total was recorded and one new FRB was discovered.

- The Parkes Multibeam Pulsar Survey (PMPS) (Keane et al., 2010, 2011). This survey targeted low Galactic latitudes and had an on-sky integration of 2115 hr. One new FRB was detected here.

- The Parkes High-latitude (PH) Survey (Burgay et al., 2006). 506 hr of total on-sky time was recorded with no new FRBs found.

- The EGU survey targeted 56 unidentified mid-Galactic latitude gamma-ray sources from the third EGRET catalogue (Hartman et al. 1999, Crawford et 
al. 2006).

- The PLMC survey is a new pulsar and transient survey of the LMC which is sensitive to millisecond pulsars in the LMC for the first time (Ridley et al. 2013).

- The SMC survey was a deep search for pulsars in the Magellanic Clouds (Crawford et al. 2001, Manchester et al. 2006, Ridley et al. 2013, Lorimer et al. 2007).

We added the $7512 \mathrm{hr}$ of time from the surveys above to the $719 \mathrm{hr}$ from our three surveys, and following the method described in $\S 3.1$, we ran a likelihood analysis to determine a statistically likely all-sky rate of detectable FRBs. From this combined survey set, we find a rate of $\mathcal{R}=3.3_{-2.2}^{+3.7} \times 10^{3}$ events per day per sky above a fluence limit of $3.8 \mathrm{Jy} \mathrm{ms}$ at the $99 \%$ confidence level. This is an improvement over the previous estimate of $\mathcal{R}=4.4_{-3.1}^{+5.2} \times 10^{3}$ events per day above a 4 Jy ms fluence limit (99\% confidence). Fig. 3.3 shows the likelihood function for both the old and new all-sky rates.

Our derived FRB event rate above a uniform fluence threshold combines the results of the rates determined individually from the 8 different Parkes surveys, while also accounting for the different single-pulse search processing methods and different telescope backends used in these surveys. Other rate estimates that have been published from Parkes observations have used only a single survey or subset of surveys (e.g., the HTRU-S survey analysed by Champion et al. 2016 and Keane \& Petroff 2015) which have a smaller total on-sky time than the combined set of surveys 
that we used. Given the large uncertainties in all of these rates (including ours), they are all compatible with each other. We note that the PMPS was conducted at low Galactic latitudes $\left(|b|<5^{\circ}\right)$, and Galactic-plane effects may significantly influence detectability of any FRBs present and hence can affect underlying FRB rate estimates.

The inclusion of this information in the future analysis of other Parkes multibeam surveys (such as the complete PLMC survey, of which only $20 \%$ has been observed and processed; Ridley et al. 2013) will help further constrain the all-sky FRB rate.

\subsection{Conclusions}

We estimated an all-sky event rate of FRBs using Bayesian analysis and applying it to all the pulsar surveys that have been searched for FRBs. We used results from six surveys and, accounting for the different backends used in each survey, total sky coverage and total observation time, we determined that the total event rate of FRBs with a fluence above $4.0 \mathrm{Jy} \mathrm{ms}$ is $\mathcal{R}=4.4_{-3.1}^{+5.2} \times 10^{3} \mathrm{FRBs}$ day $^{-1} \mathrm{sky}^{-1}$ at the 99\% confidence level. Three additional archival Parkes surveys were searched later and found no FRBs. We incorporated these results and using the same Bayesian analysis, modified our event rate of FRBs. This resulted in an even lower all-sky

rate of $\mathcal{R}=3.3_{-2.2}^{+3.7} \times 10^{3}$ events per day per sky above a fluence limit of $3.8 \mathrm{Jy} \mathrm{ms}$ at the $99 \%$ confidence level. This result led us to conclude that lack of detections at intermediate latitudes are likely to be due to a lower FRB rate as compared to the 


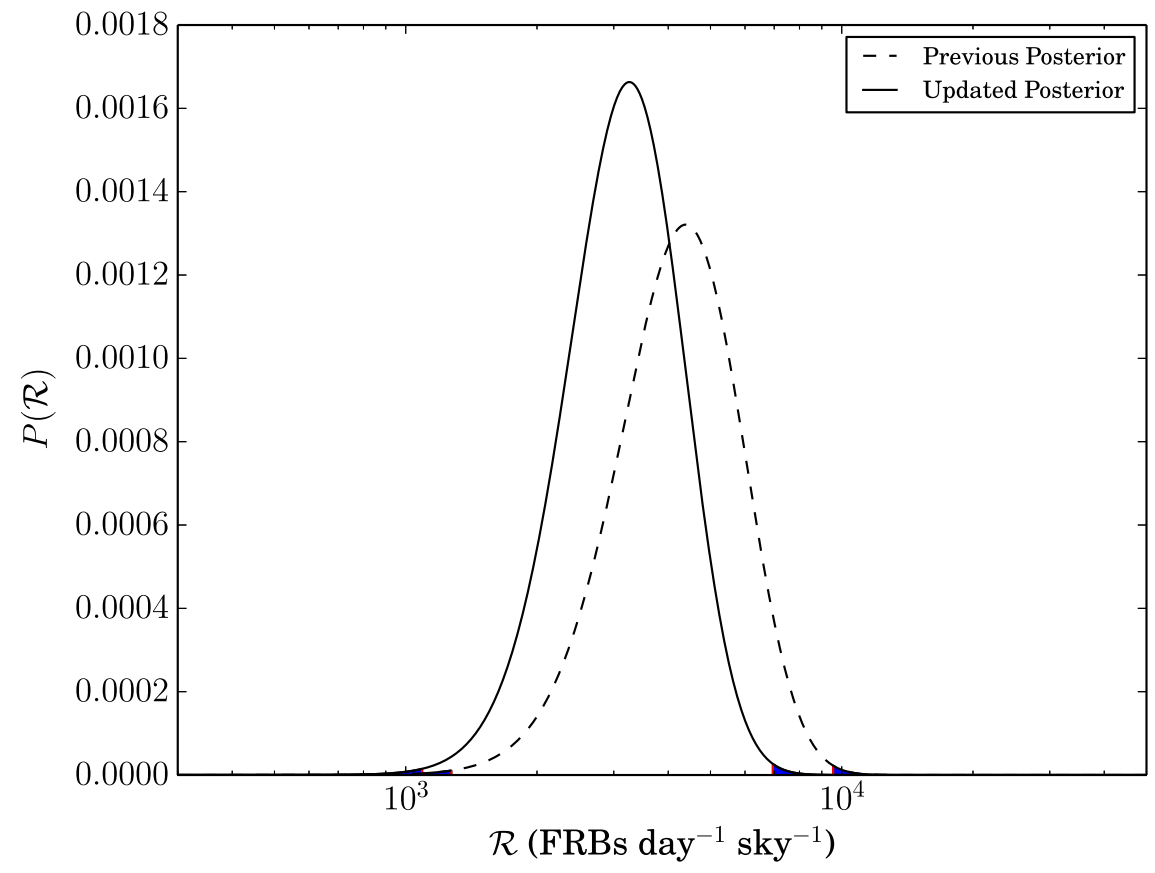

Figure 3.3: The posterior probability density function of the event rate of Parkesdetectable FRBs, determined from the 5 Parkes surveys (totaling $7512 \mathrm{hr}$ ) analyzed in $\S 3.4$ (dashed curve) and from the addition of the 3 Parkes surveys (totaling $719 \mathrm{hr}$ ) described in $\S 3.5$ (solid curve). All surveys were searched to high DMs (at least $3000 \mathrm{pc} \mathrm{cm}^{-3}$ ). The rate analysis considered the different single-pulse search processing methods and observing backends used in the different surveys. The resulting new all-sky FRB rate is $\mathcal{R}=3.3_{-2.2}^{+3.7} \times 10^{3}$ events per day per sky above a fluence limit of $3.8 \mathrm{Jy} \mathrm{ms}$ at the $99 \%$ confidence level. 
rate estimates done previously ( 10000 FRBs per day per sky) and does not necessarily imply a dearth of FRBs at intermediate latitudes as proposed by (Petroff et al., 2014). Additionally, we predicted the number of expected discoveries in future planned surveys. 


\section{Chapter 4}

Searching for host galaxies of potentially extragalactic RRATs

The work in this chapter was originally published as: Akshaya Rane and Avi Loeb, 'Searching for host galaxies of potentially extragalactic RRATs', MNRAS Letters, volume 467, L11-L15, 2017.

\subsection{Introduction}

FRBs are millisecond-duration bright radio bursts, first reported in 2007 (Lorimer et al., 2007). FRBs have dispersion measures that are greater than the maximum Galactic value expected in their direction. All of the FRBs published so far (see Chapter 1) have been detected between $0.7-1.5 \mathrm{GHz}$ and only one of them has been observed to repeat. Recently, a host galaxy has been confirmed for this repeating FRB 121102 at a redshift of 0.19 (Tendulkar et al. 2017, Marcote et al. 2017, Chatterjee et al. 2017). This association confirms FRBs as extragalactic provided other FRBs are similar to this one. Another possible repeat event as inferred by Maoz et al. 2015 to be originating from the same source is still under debate since the positions are consistent but the DMs are very different. Fourteen of these sources have been detected at high Galactic latitudes $(|b|>5)$ and their large dispersion measures $\left(\mathrm{DM} \sim 375-1700 \mathrm{pc} \mathrm{cm}^{-3}\right)$ exceed the expected Galactic contribution predicted by the NE2001 model in the direction of the bursts (Cordes \& Lazio, 
2002), suggesting an extragalactic origin.

Since the telescopes that are efficient in detecting these bursts are single-dish, the beam sizes are typically a few arcminutes which makes the position uncertainty large, limiting the ability to identify the host galaxy. The excess DM suggests redshifts in the range $\sim 0.3-1.3$ for FRBs if it originates from the intergalactic medium. Given these circumstances, it is extremely difficult to associate an FRB with a particular host galaxy unless there is some extraordinary evidence for coincident, transient multiwavelength emission within the beam or if it repeats and follow-up interferometric observations pinpoint the host galaxy similar to FRB 121102.

In view of the lack of any extragalactic counterparts identified so far, a number of other scenarios remain equally intriguing. Loeb et al. (2014) considered the case of nearby Galactic flaring main-sequence stars within $1 \mathrm{kpc}$ as the sources of FRBs. They propose that the excess dispersion comes from propagation through the stellar corona. However Luan \& Goldreich (2014) argued that the free-free absorption would conceal any radio signal emitted from below the corona. Also, FRBs exhibit quadratic dispersion curves that are consistent with the assumption of weak dispersion in a low density plasma. So, for the above model, in which the dispersion is concentrated in a relatively high density region, the quadratic dispersion approximation breaks down as the plasma frequency is comparable to the propagation frequency, posing significant problems for this model (Dennison, 2014). However, Maoz et al. (2015) have recently found possible flare stars in additional FRB fields using time-domain optical photometry and spectroscopy. The authors have evaluated the chance probabilities of these possible associations to be in the 
range $0.1 \%$ to $9 \%$. FRB 140514 was discovered in the radio follow-up observations of FRB 110220, three years apart within the same radio beam. Maoz et al. (2015) also claim that these two FRBs are from the same repeating source with $99 \%$ confidence and are consistent with the flare-star scenario with a varying plasma blanket between bursts. More FRB detections in general are necessary to confirm or refute a Galactic origin of at least a subset of FRBs.

Since pulses from RRATs are largely indistinguishable from FRBs, as explained in detail in $\S 4.2$, we decided to find and confirm the origin of a subset of RRATs that appear to be close to the edge of the galaxy. Taking into account the uncertainty in the free electron density model, these RRATs could possibly have an extragalactic origin. The excess DM for our sample is not as high as seen in FRBs, corresponding to very low redshifts $(z<0.01)$. For example, a redshift of 0.001 corresponds to an angular diameter distance of $\sim 4.3 \mathrm{Mpc}$. The size of a nearby host galaxy (diameter $\sim 25 \mathrm{kpc}$ ) at this redshift is $\sim 16^{\prime}$, comparable to the beam size of a single-dish telescope (for example, the Parkes beam width is $14^{\prime}$ ). This similarity of scales would make the association, if found, more definitive than at cosmological distances, where many galaxies are found within the telescope beam.

Before it was discovered that FRB 121102 is repeating, Keane et al. (2016) discussed the uncertainty in the RRAT/FRB classification by analysing RRATs from which only one pulse has been detected so far. Although, multiple bursts have been detected for FRB 121102, no period is yet been confirmed. This is similar to some of the RRATs for which finding an unique period is extremely difficult if the pulse has too many components or if the period is very small. The only clear distinction is 
then associated with their DM values as of now. If there is any additional difference in RRATs and FRBs related to their intrinsic spectral properties, then in that case, finding a host galaxy can really help in order to constrain emission models for these bursts.

In $\S 4.2$ we compare the most commonly used model for the Galactic distribution with a newly available model to derive the DM distribution of radio transients on the sky. In $\S 4.3$ we outline the basic criterion used to choose our RRAT sample. The results for each RRAT candidate are discussed in $\S 4.4$. In $\S 4.5$ we discuss the non-detection of host galaxies and related probabilities. Finally, in $\S 4.6$ we summarize our results and present our conclusions.

\subsection{Galactic free electron density models}

The NE2001 model (Cordes \& Lazio, 2002) describes the structure of ionised gas in the Galaxy and is widely used to estimate distances to radio pulsars for which DM is the only distance indicator. In the case of FRBs for which the DMs are too high, this model is used to estimate the DM contribution in the direction of FRB from the Galaxy. This model is based on the observed DMs of Galactic radio pulsars and includes contributions from the thin disk associated with low-latitude HII regions, the thick-disk, the spiral arms, small-scale features corresponding to local ISM, individual high-density clumps and voids. However, the uncertainty in the NE2001 model could be more than $\sim 20 \%$, particularly at higher latitudes, as also discussed in Gaensler et al. (2008). 
Recently, a new model by Yao, Manchester and Wang (Yao et al., 2016), called as YMW16 has been proposed for the distribution of free electrons in the Galaxy, the Magellanic Clouds, and the inter-galactic medium (IGM). This model is based on measurements from 189 pulsars with independently determined distances. We compared the two models by integrating both models to the edge of the Galaxy for each radio transient's direction. The list of pulsars in the Milky Way, SMC and the LMC is obtained from Manchester et al. (2005). The ratio $r$ of the measured DM to the maximum Galactic DM versus the measured DM is plotted for the NE2001 model in Spitler et al. (2014) and is reprinted here (see Figure 4.1. We show a similar plot for the YMW16 model in Figure 4.2. If we compare the two plots, we can see that the galactic DM contribution is lowered along certain lines of sight towards the galactic center (GC) pulsars with the newer model, minimizing the gap between GC pulsars and the overall pulsar population. Pulsars in the LMC and SMC and FRBs all have $r>1$, consistent with the NE2001 model. A small fraction of pulsars have $r>1$ in both models, possibly due to uncertainties or them being in the Galactic halo but we will not discuss these pulsars here. Another promising difference between the two models is that all RRATs had $r<1$ according to the NE2001 model, thus confirming their Galactic origin; however, according to the YMW16 model, some RRATs now have shifted to having $r$ slightly above 1. Assuming this model is closer to the true values than the NE2001 model, these RRATs are very similar to some FRBs. Thus this RRAT sample provides a promising opportunity to find host galaxies that are close to us since their excess DM is much lower than most of the FRBs. We also compared the pulse widths, fluxes, and r-values for both 


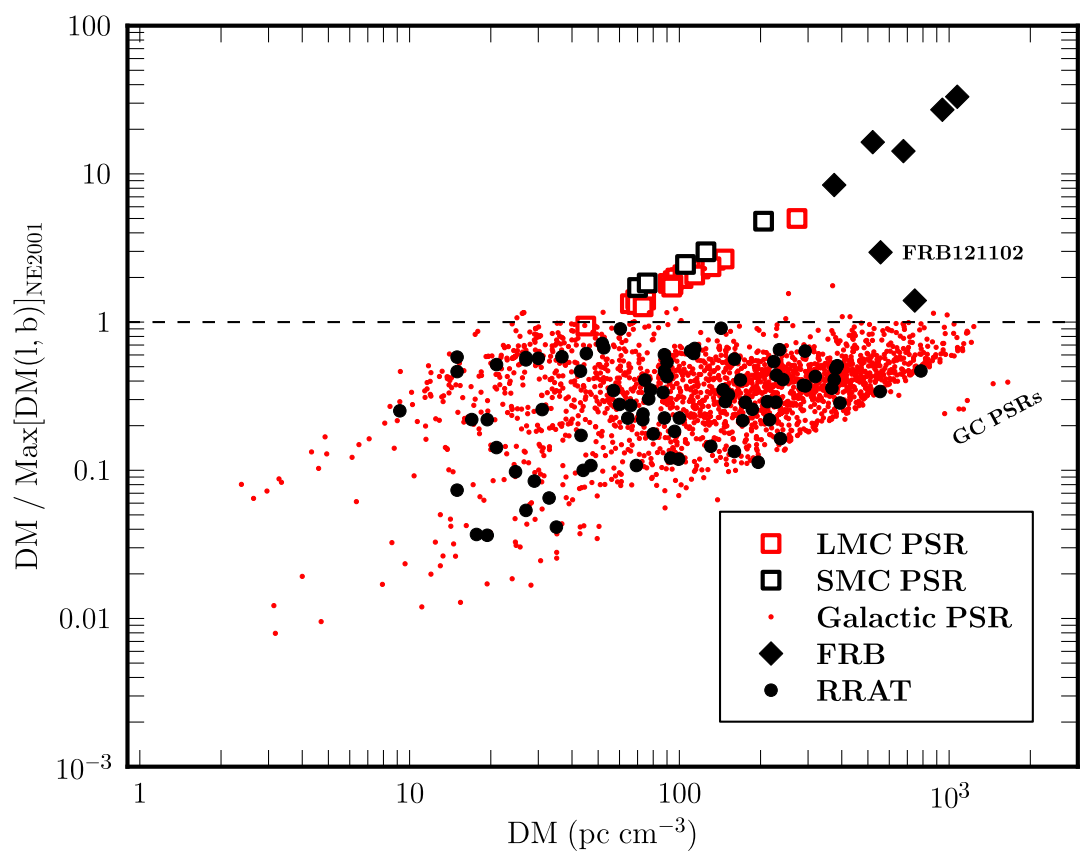

Figure 4.1: The ratio of measured DM to maximum Galactic DM versus the measured DM (in pc $\mathrm{cm}^{-3}$ ) for all radio transients. The maximum Galactic DM is calculated by integrating the NE2001 model to the edge of the Galaxy for each transient's direction. The dashed line shows the maximum ratio expected for Galactic objects if the electron density is accurate for all lines of sight. 


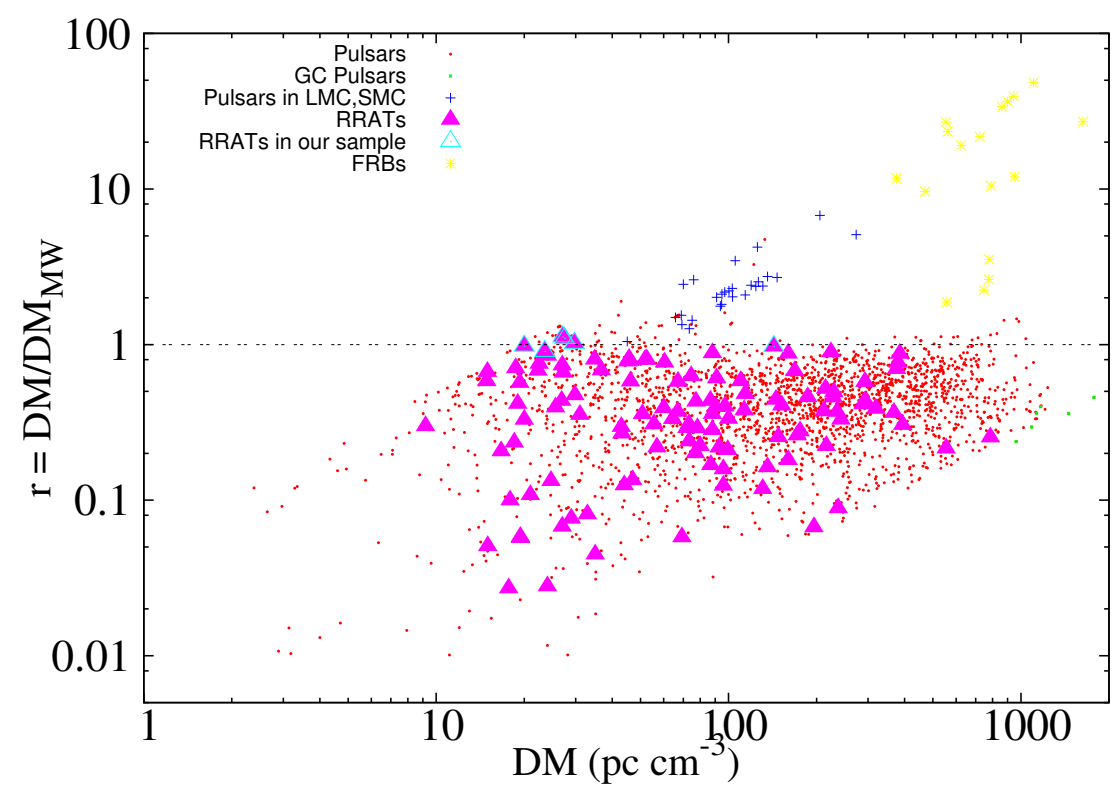

Figure 4.2: The ratio of measured DM to maximum Galactic DM versus the measured DM (in pc $\mathrm{cm}^{-3}$ ) for all radio transients. The maximum Galactic DM is calculated by integrating the YMW16 model to the edge of the Galaxy for each transient's direction. The dashed line shows the maximum ratio expected for Galactic objects if the electron density is accurate for all lines of sight.

RRATs and FRBs, as seen in Figure 4.3. The two sample Kolmogorov-Smirnov test measures the greatest distance $D$ between the cumulative distribution functions of the two sample distributions and gives a probability that the two samples are drawn from the same parent distribution described as a $p$-value. We got $\mathrm{D}=0.37$ and a $\mathrm{p}$ value of 0.03 for the pulse widths, thus indicating that the pulse width distributions of the two populations are not significantly different. For the flux and r-values distribution, we got $\mathrm{D}=0.80,0.82$ and smaller $\mathrm{p}$-values $\left(9.10 \times 10^{-9}, 1.22 \times 10^{-9}\right.$ respectively). This is not surprising since the peak fluxes and r-values of known FRBs are higher than RRATs. 

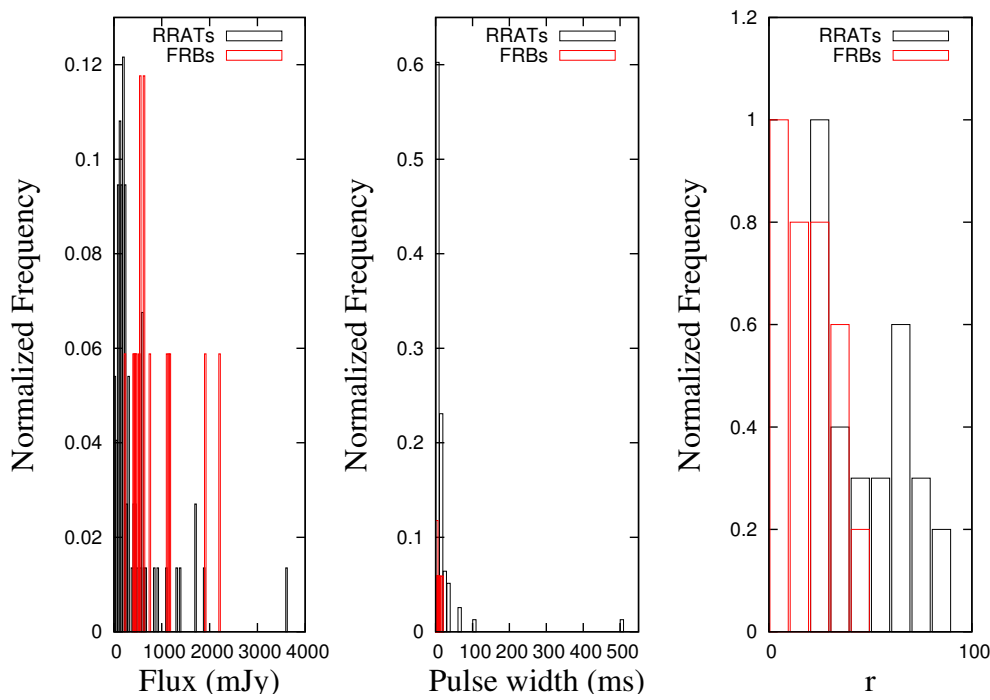

Figure 4.3: The normalized histograms of peak fluxes, pulse widths, and the ratio of measured DM to maximum Galactic DM versus the measured DM for RRATs and FRBs.

\subsection{RRAT sample}

In an attempt to find host galaxies as discussed in $\S 4.1$, we have selected a sample of RRATs which have $r$ greater than 0.9 and for which $\mathrm{DM}_{\text {diff }}=\mathrm{DM}-\mathrm{DM}_{\mathrm{MW}}$ is less than $10 \mathrm{pc} \mathrm{cm}^{-3}$ as seen in Figure 4.4. These RRATs may be at the edge of our galaxy. The second criterion is imposed so that the measured DM value is within the uncertainty of the electron density model. Both the criteria therefore make these RRATs either Galactic or extragalactic.

\subsection{Results}

Next, we discuss the individual RRAT candidates which could possibly be FRBs. If the DM due to the host galaxy is neglected and if the excess $\mathrm{DM}_{\text {diff }}$ is assumed to be entirely due to the intergalactic medium, then we infer a redshift $z \sim 0.005$ and distances up to $\sim 20 \mathrm{Mpc}$. But since these RRATs have low DM and 


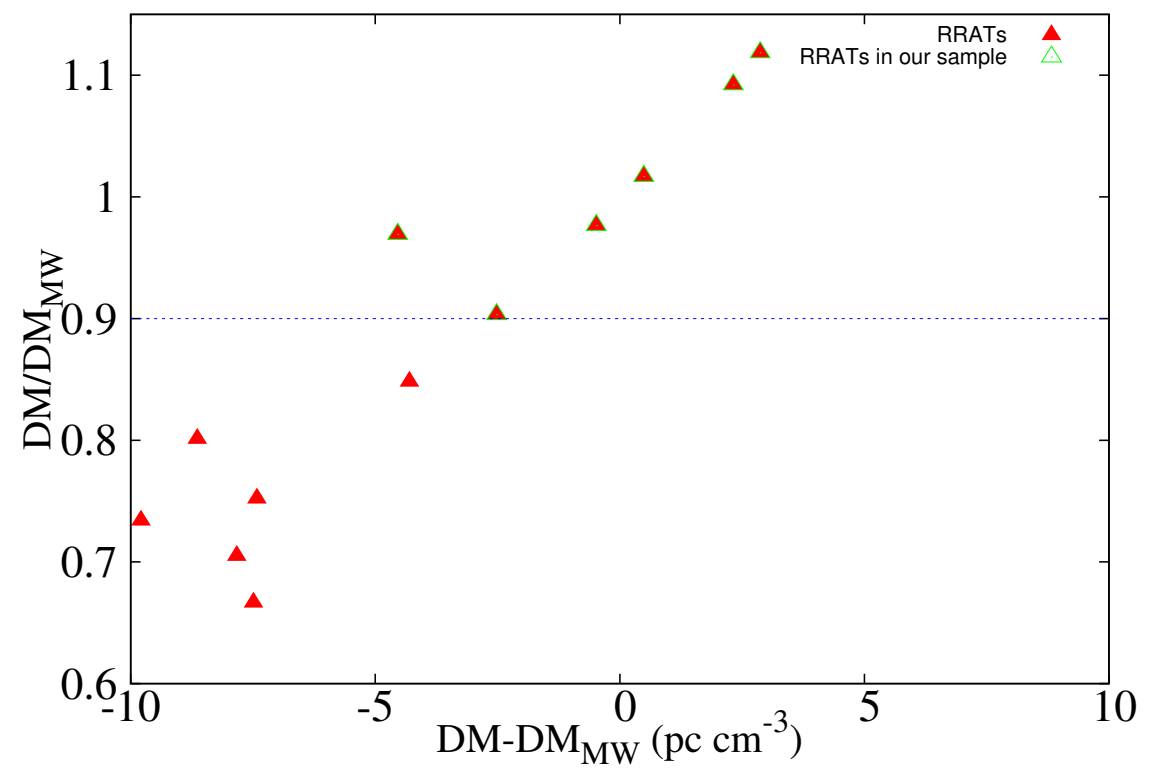

Figure 4.4: A zoomed in plot of the ratio between the measured DM and the maximum Galactic DM versus measured DM subtracted from maximum Galactic DM for RRATs.

the Galactic $\mathrm{DM}_{\mathrm{MW}}$ uncertainties might be within this excess, we search for galaxies within $120 \mathrm{Mpc}$ (corresponding to a $\mathrm{DM} \sim 30 \mathrm{pc} \mathrm{cm}^{-3}$ for the local density of the intergalactic medium) at the corresponding beam size based on the redshift information provided on $\mathrm{NED}^{1}$ and the GLADE catalog which is exclusively constructed for nearby galaxies. Since this RRAT sample which has no sources with $|b|<5$, the galaxy catalogs being incomplete is not concerning. The summary is given in Table 4.1. The $\mathrm{DM}_{\text {halo }}$ contribution is determined from the free electron density profile as a function of galecto-centric radius obtained by the latest model that fits best O VIII observations (see Figure 8 of Miller \& Bregman 2015). The number of objects found in NED within this search radius are listed in each subsection, and if the spectrum is available then their redshifts are determined by cross-correlating the

\footnotetext{
${ }^{1}$ The NASA/IPAC Extragalactic Database (NED) is operated by the Jet Propulsion Laboratory, California Institute of Technology, under contract with the National Aeronautics and Space Administration.
} 


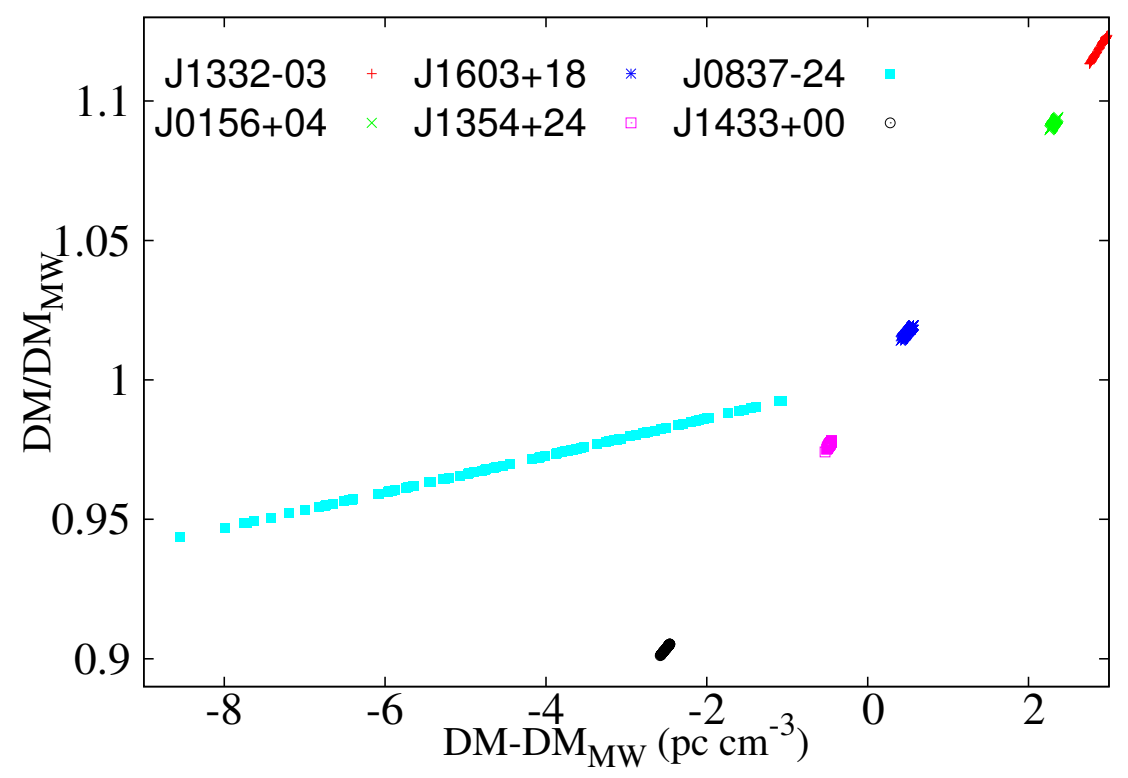

Figure 4.5: The ratio of measured DM to maximum Galactic DM plotted against the difference in measured DM and maximum Galactic DM for each RRAT in our sample within the beam uncertainty.

spectrum against template spectra using the IRAF task XVSAO in the RVSAO package (For list of templates, see http://tdc-www.harvard.edu/iraf/rvsao/Templates/).

We also determined the variation in $\mathrm{DM}_{\mathrm{MW}}$ within the beam uncertainty using the YMW16 model for each RRAT, as seen in Figure 4.5. The $\mathrm{DM}_{\mathrm{MW}}$ variation is within $12 \%$ for all six RRATs.

\subsubsection{RRAT J1332-03}

This RRAT was discovered in the 350-MHz Drift-scan pulsar survey with the GBT and was confirmed in follow-up observations and the period was determined as well (Karako-Argaman et al., 2015). The uncertainty in the beam position is 19.4 ' at $350 \mathrm{MHz}$. The four extra-galactic source galaxies found within this search radius are listed in Table 4.2. For LCRS B133012.2-031854, we did not find any 
Table 4.1: The subset of RRATs included in our sample. Columns 1 to 5 list the RRAT name, Galactic longitude and latitude, uncertainty in position $\delta$, and measured DM, as obtained from the RRATalog. Column 6 lists the Galactic contribution to the DM, column 7 lists the ratio of measured DM to the maximum Galactic DM along that line of sight. Column 8 lists contribution to the DM from the Galactic halo and column 9 reports number of extragalactic objects seen within the search radius of beam uncertainty. The search was carried out in NED out to $120 \mathrm{Mpc}$.

\begin{tabular}{ccccccccc}
\hline $\begin{array}{c}\text { Name } \\
\text { RRAT }\end{array}$ & $\begin{array}{c}l \\
(\mathrm{o})\end{array}$ & $\begin{array}{c}b \\
(\mathrm{o})\end{array}$ & $\begin{array}{c}\delta \\
(')\end{array}$ & $\begin{array}{c}\mathrm{DM} \\
\left(\mathrm{pc} \mathrm{cm}^{-3}\right)\end{array}$ & $\begin{array}{c}\mathrm{DM}_{\mathrm{mw}} \\
\left(\mathrm{pc} \mathrm{cm}^{-3}\right)\end{array}$ & $\begin{array}{c}\mathrm{DM}_{\text {halo }} \\
\left(\mathrm{pc} \mathrm{cm}^{-3}\right)\end{array}$ & $N_{\text {obj }}$ \\
\hline J1332-03 & 322.25 & 57.91 & 19.4 & 27.1 & 24.23 & 1.12 & 0.50 & 4 \\
J0156+04 & 152.00 & -55.00 & 7.5 & 27.5 & 25.18 & 1.09 & 0.50 & 1 \\
J1603+18 & 32.85 & 45.28 & 7.5 & 29.7 & 29.21 & 1.02 & 0.50 & 0 \\
J1354+24 & 27.43 & 75.78 & 19.4 & 20.0 & 20.48 & 0.98 & 0.07 & 0 \\
J0837-24 & 247.45 & 9.80 & 7.0 & 142.8 & 147.30 & 0.97 & 0.22 & 1 \\
J1433+00 & 349.75 & 53.79 & 7.5 & 23.5 & 26.02 & 0.90 & 0.06 & 1 \\
\hline
\end{tabular}

spectrum from the online literature. All of these galaxies have higher redshifts than what we would require to account for the intergalactic medium contribution. Hence these galaxies are most probably not related to this RRAT.

\subsubsection{RRAT J0156+04}

This RRAT was discovered in the single-pulse search of the data obtained in the Arecibo Drift Pulsar survey at $327 \mathrm{MHz}$ and two pulses were observed from it at only one epoch (Deneva et al., 2016). Follow-up observations detected no pulses from this RRAT. The uncertainties in both the coordinates are $7.5^{\prime}$, the $327 \mathrm{MHz}$ beam radius. We found one galaxy within this beam with a redshift of 0.18 , higher than what would be expected, hence indicating no association with this RRAT (Table 4.2). 


\subsubsection{RRAT J1603+18}

This RRAT was also discovered in the Arecibo Drift Pulsar survey at $327 \mathrm{MHz}$ and was confirmed in follow-up observations and the period was determined as well (Deneva et al., 2016). We did not find any galaxy within a search radius of $7.5^{\prime}$ from the beam center up to $120 \mathrm{Mpc}$.

\subsubsection{RRAT J1354+24}

This RRAT was discovered in the Green Bank North Celestial Cap survey (GBNCC) at $350 \mathrm{MHz}$ (Karako-Argaman et al., 2015). We did not find any galaxy within a search radius of $19.4^{\prime}$ up to $120 \mathrm{Mpc}$.

\subsubsection{RRAT J0837-24}

This RRAT was discovered in the single-pulse search of the High Time Resolution Universe (HTRU) pulsar survey carried out with the Parkes telescope at 1.4 GHZ (Burke-Spolaor et al., 2011). We found one galaxy within the beam radius of $15.0^{\prime}$ of Parkes. We could not find a spectrum for this galaxy and hence the redshift is determined using the luminosity-size relation (see equation 4 of McIntosh et al. 2005). The inferred redshift of 0.15 is too high, indicating no association with this RRAT (Table 4.2). 


\subsubsection{RRAT J1433+00}

This RRAT was discovered in the Arecibo Drift Pulsar survey at $327 \mathrm{MHz}$ and was confirmed in follow-up observation (Deneva et al., 2016). The 2dFGRS source found within the search radius of $7.5^{\prime}$ was at an inferred redshift of 0.07 , higher than what would be expected, so it is unrelated to the RRAT (see Table 4.2). 


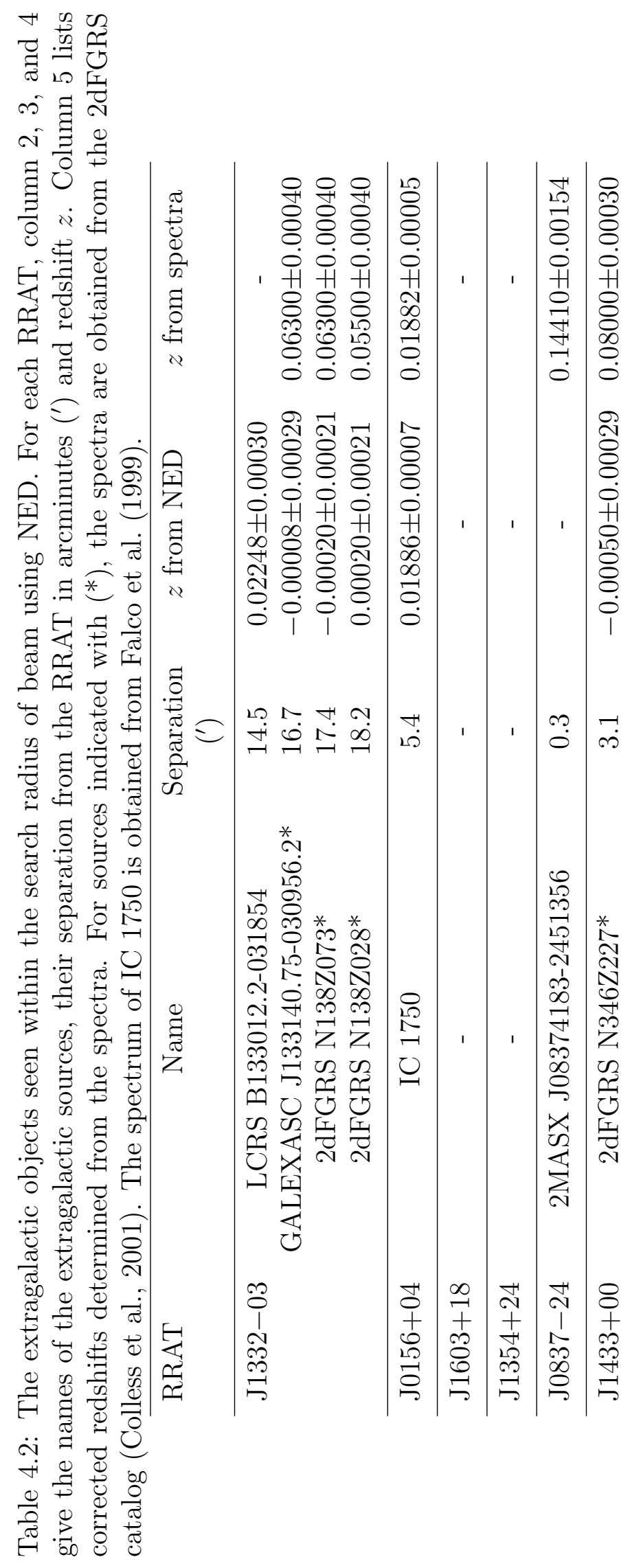




\subsection{Discussion}

Since we did not find any plausible host galaxy that might be associated with any of the six RRATs in our sample, these most likely do not have an extragalactic origin. For RRAT J1603+18, the $\mathrm{DM}_{\text {halo }}$ contribution adds to match the measured DM and hence this RRAT might be within the halo of our galaxy. We have also determined the DM associated with the local group by checking the direction of each of these RRATs based on the right panel of Figure 3 in Rubin \& Loeb (2014), which yields a DM $\sim 5 \mathrm{pc} \mathrm{cm}^{-3}$. RRATs J1332-03 and J0156+04 could reside within the local group.

The probability of finding a galaxy within the volume out to $120 \mathrm{Mpc}$ by chance for a beam radius of $7.5^{\prime}$ is $\sim 0.043$, whereas for a beam radius of $19.4^{\prime}$ it is $\sim 0.28$, based on the average number density of galaxies within the search volume using NED. Our null result is thus consistent with this estimate.

\subsection{Summary and conclusions}

We have presented a search for host galaxies in a subset of RRATs that are at the edge of our Galaxy. These RRATs are interesting since they could either have Galactic or extragalactic origin. In the latter case, the sizes of the host galaxies on the sky at such distances would be comparable to the beam size of a single-

dish telescope. We did not find any nearby host galaxy associated with the six RRATs in our sample. Although finding nearby galaxies for such RRATs that could possibly be FRBs is a novel approach, the probability of actually finding a nearby 
host galaxy is low. Nevertheless, we suggest applying this search strategy to new discoveries of RRATs that will be in the uncertainty zone of the electron density model. Follow-up observations could determine if these RRATs are of a Galactic origin or are extragalactic FRBs, and help us pin down the mysterious origin of FRBs. 


\section{Chapter 5}

\section{Empirical Population Models for Fast Radio Bursts}

The work in this chapter will be submitted to MNRAS as: A. Rane, D. R. Lorimer, J. Newman, K. Ponder, S. K. Sarbadhicary, B. Zhang, D. Palaniswamy, 'Empirical Population Models for Fast Radio Bursts'.

\subsection{Introduction}

As discussed earlier in this thesis, FRBs are very bright, short millisecond bursts of radio waves that are highly dispersed. Due to the short duration of FRBs (typical pulse widths are $0.3-16.0 \mathrm{~ms}$ ), the emission regions of these exotic astrophysical events must be small. For all 23 bursts published to date (Lorimer et al. 2007; Keane et al. 2012; Thornton et al. 2013; Spitler et al. 2014; Burke-Spolaor \& Bannister 2014; Ravi et al. 2014; Petroff et al. 2015a; Masui et al. 2015; Ravi et al. 2016; Keane et al. 2016; Champion et al. 2016; Caleb et al. 2017), the dispersion delay with observing frequency $\nu$ follows a $\nu^{-2}$ frequency dependence and the scattering indices, when measurable, follow $\nu^{-4}$ frequency dependence, similar to pulsars, both of which are consistent with theoretical values for a pulse traveling through a cold turbulent plasma of free electrons. However, the very high DM, in the range $260-1630 \mathrm{~cm}^{-3} \mathrm{pc}$, are more than an order of magnitude larger than the 
DM contribution expected from the interstellar medium of the Milky Way in these directions. This is highly suggestive of an extragalactic origin.

Although a variety of models have been proposed to explain the physical origin of FRBs (see $\S 1.4 .3$ ), the true progenitor source (or sources) remains unknown. Theories for the progenitors of FRBs must explain the large DMs and seemingly high energy output and must be consistent with the very high FRB event rates inferred from current observations (see Chapter 3). With only a small sample size of FRBs, many of their properties are consistent with a number of progenitor models. Many follow-up observations have been carried out to search for repeating bursts at the location of known FRBs and to see if there is emission at other wavelengths.

So far, only one FRB, 121102, has been observed to repeat (Spitler et al., 2014, 2016; Scholz et al., 2016). This discovery demonstrated that its source survives the energetic bursts and ruled out models involving cataclysmic events. But since none of the other FRBs have been observed to repeat so far, other models cannot be ruled out completely as of now. Additionally, the other 17 FRBs were discovered with single-dish telescopes with a beamsize of a few arc-minutes (e.g., $14^{\prime}$ for the Parkes telescope). This makes the localization extremely difficult to determine. The followup observation of FRB 121102 with the interferometric Karl Jansky Very Large Array (VLA) recently localized it at sub-arcsecond resolution and located its starforming host galaxy at a redshift of $\approx 0.19$ (Tendulkar et al., 2017) and determined association with a persistent and compact radio source (Chatterjee et al., 2017). The properties are consistent with two scenarios based on the data from this burst. Marcote et al. (2017) argue that the source could be associated with either a low- 
luminosity active galactic nuclei or a young supernova remnant powered by a highly energetic neutron star or a magnetar (Metzger et al., 2017). This FRB, however, is unique compared to the wider population of FRBs and hence it is insufficient to claim that all FRBs might be originating in the low-redshift universe similar to FRB 121102.

In this chapter, we present simulations of a cosmological population of FRBs to constrain the possible physical scenarios for the origin.

Previously, a number of studies have been carried out to simulate the FRB population to study the observable properties of FRBs, for example, dispersion measure, flux densities, and pulse widths. Dolag et al. (2015) studied the distribution and energetics of FRBs using cosmological hydrodynamic simulations. They showed that the observations are consistent with FRBs being standard candles and found a constant isotropic burst energy of $\sim 7 \times 10^{40}$ ergs. Caleb et al. (2016) assumed a log-normal intrinsic energy distribution of FRBs and compared the cosmological simulated population with the nine FRBs discovered in the HTRU high latitude pulsar survey (Thornton, 2013; Champion et al., 2016). The FRBs were considered to be uniformly distributed over a co-moving volume and also following the cosmic star formation history. The cumulative number of sources $N$ within some volume corresponding to a distance $d$ that are detectable above some flux threshold $S$ is

$$
N(>S) \equiv N(<d)=\frac{4 \pi}{3} n d^{3}
$$


where $n$ is the number density. If all sources have same intrinsic luminosity $L$ then

$$
S=\frac{L}{4 \pi d^{2}}
$$

substituting for $d$ gives

$$
N(>S) \propto S^{-3 / 2} \propto S^{\alpha}
$$

Here $\alpha$ is the power law index which has the value $-3 / 2$ in this simple model. Caleb et al. (2016) determined $\alpha$ to be $-0.9 \pm 0.3$. Oppermann et al. (2016) investigated the distribution of observed flux densities with a simulated population and found that they are consistent with a constant number density of FRBs in Euclidean space. These authors also constrained the spectral power law index of FRBs $\alpha$ to be between -0.8 and -1.7 which is consistent with the results from Caleb et al. (2016).

In another study, Bera et al. (2016) assumed the spectral energy density depending on some emission profile for FRB. They used FRB 110220 as a reference event for their analysis. They also used two models to account for the scatter broadening arising from the IGM. The first model is based on the empirical relation between the width and the DM due to the ISM and rescaling it for scattering in the IGM. The second model is a theoretical model in which the amount of temporal smearing by the IGM is estimated by analyzing the contributions to the scattering measure (explained in $\S 1.3$ ) from the diffuse IGM, intervening galaxies, and intracluster gas (Macquart \& Koay, 2013). Additionally, some population studies have 
been focused on very specific quantities such as DM in the host galaxy. Yang \& Zhang (2016) performed Monte Carlo simulations by generating a redshift distribution of FRBs to infer the host galaxy DM which is a poorly known parameter. A study by Xu \& Han (2015) simulated the spatial distribution of FRBs in a host galaxy and calculated the DM along the sightlines to the edge of host galaxies including spiral, elliptical, and dwarf galaxies at different inclination angles and found a mean value of $70 \mathrm{pc} \mathrm{cm}^{-3}$.

We start with similar assumptions about the spatial distribution of FRBs. We assume that FRBs are uniformly distributed in space and, like many studies of the pulsar populations (e.g., Lorimer et al., 1993), we do not consider beaming of the FRBs specifically here. Instead, our simulations pertain to the potentially observable population. We use three distributions for energy and luminosity to generate FRB events in our simulations. The redshift distribution is drawn from their comoving density. The dispersion measures are derived using the properties of the ISM, IGM, and the host galaxy. We produce energy, redshift, peak flux, DM, effective width, and signal-to-noise ratio $\mathrm{S} / \mathrm{N}$ for each simulated FRB and compare this population to the observed FRBs. The analysis of the models is discussed in $\S 5.10$.

\subsection{Comparison sample}

The goal of this study is to model the sample of FRBs found at the Parkes telescope so far using the multi-beam receiver. Specifically, we consider the Parkes 
Table 5.1: The Galactic longitude $l$, Galactic latitude $b$, DM, observed pulse width $w_{\text {obs }}$, observed peak fluxes $S_{\text {peak,obs }}$, and the survey references of the known FRB sample used in our analysis.

\begin{tabular}{lrcrrrr}
\hline FRB & $\begin{array}{c}l \\
\left({ }^{\circ}\right)\end{array}$ & $\begin{array}{c}b \\
\left({ }^{\circ}\right)\end{array}$ & $\begin{array}{c}\text { DM } \\
\left(\mathrm{cm}^{-3} \mathrm{pc}\right)\end{array}$ & $\begin{array}{c}w_{\text {obs }} \\
(\mathrm{ms})\end{array}$ & $\begin{array}{c}S_{\text {peak,obs }} \\
(\mathrm{Jy})\end{array}$ & Reference \\
\hline 010125 & 356.6 & -20.0 & 790 & 10.6 & 0.5 & Burke-Spolaor \& Bannister (2014) \\
010724 & 300.7 & -41.8 & 375 & 16.0 & 0.5 & Lorimer et al. (2007) \\
090625 & 226.4 & -60.0 & 899 & 1.9 & 1.1 & Champion et al. (2016) \\
110220 & 50.8 & -54.8 & 944 & 6.6 & 1.1 & Thornton et al. (2013) \\
110626 & 355.9 & -41.8 & 723 & 1.4 & 0.6 & Thornton et al. (2013) \\
110703 & 81.0 & -59.0 & 1104 & 3.9 & 0.5 & Thornton et al. (2013) \\
120127 & 49.3 & -66.2 & 553 & 1.3 & 0.6 & Thornton et al. (2013) \\
121002 & 308.2 & -26.3 & 1629 & 5.4 & 0.4 & Champion et al. (2016) \\
130626 & 7.5 & 27.4 & 952 & 2.0 & 0.7 & Champion et al. (2016) \\
130628 & 226.0 & 30.7 & 470 & 0.6 & 1.9 & Champion et al. (2016) \\
130729 & 324.8 & 54.7 & 861 & 15.6 & 0.2 & Champion et al. (2016) \\
131104 & 260.5 & -21.9 & 779 & 2.4 & 1.2 & Ravi et al. (2014) \\
140514 & 50.8 & -54.6 & 563 & 2.8 & 0.5 & Petroff et al. (2015a) \\
150807 & 336.7 & -54.4 & 267 & 0.4 & 12.2 & Ravi et al. (2016) \\
\hline
\end{tabular}

HTRU survey (Thornton et al., 2013; Thornton, 2013). The observed quantities of these FRBs are listed in Table 5.1. The parameter space covered by this observed sample is plotted in Figure 5.1. Most of the FRBs discovered with the Parkes telescope are at high latitudes. At low latitudes, the sky temperature is enhanced and excessive scattering is observed with increased dispersive smearing which can explain fewer detections reported for observed population so far (Macquart \& Johnston, 2015). Taking into account these factors, we consider only the high latitude FRBs in our simulations. 

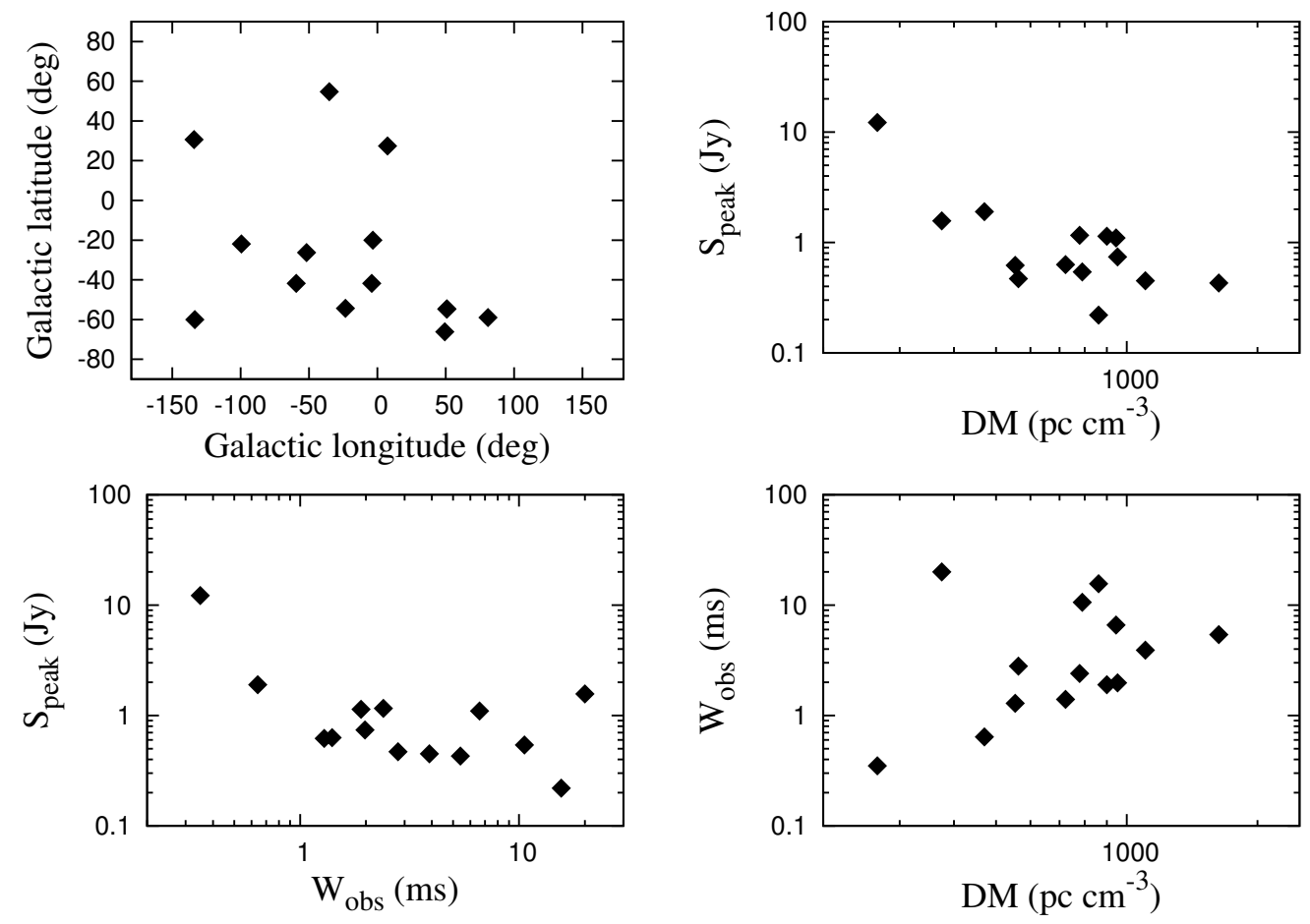

Figure 5.1: Scatter plots showing the parameter space for the observed FRB sample considered in this study. The relevant data are listed in Table 5.1.

\subsection{Energy and luminosity distributions}

We assume the intrinsic energy distribution to be either a constant or a Gaussian distribution around zero mean with some standard deviation $\sigma_{\mathrm{E}}$. The luminosity distribution is assumed to be either a power law or a Gaussian distribution around zero mean with some standard deviation $\sigma_{\mathrm{L}}$, which is a free parameter. For the power law distribution where the probability of having a luminosity in the range $L \rightarrow L+d L$, we use

$$
p(L) d L=\frac{\alpha_{L}-1}{L_{\min }}\left(\frac{L}{L_{\min }}\right)^{-\alpha_{L}} d L .
$$


Here $L_{\text {min }}$, the minimum luminosity, and the power law index $\alpha_{L}$, are both free parameters. In the simulations, $L$ and $E$ values are drawn from one of these distributions. A more detailed discussion about how these distributions are incorporated in the simulations is given in $\S 5.8$.

\subsection{Redshift distribution}

The highest inferred redshift of an FRB is about 1.3 for FRB 121002 which has a DM of $1629 \mathrm{~cm}^{-3} \mathrm{pc}$ (Champion et al., 2016). We generate events up to a redshift of 2.5 which is more than sufficient to sample the dispersion measure space of the known FRBs. We adopt a $\Lambda-\mathrm{CDM}$ model (explained in $\S 1.6 .9$ ) and follow results from the Planck Collaboration et al. (2016) with the total energy density $\Omega_{\Lambda}=0.68$, total matter density $\Omega_{m}=0.32$, and the Hubble constant $H_{0}=68.0 \mathrm{kms}^{-1} \mathrm{Mpc}^{-1}$. The maximum co-moving radial distance corresponding to a redshift of 2.5 is 5.889 Gpc, obtained using the online cosmological calculator by Ned Wright. ${ }^{1}$ The comoving volume at a redshift of 2.5 is

$$
V_{2.5}=V_{\max }=\frac{4}{3} \pi(5.889)^{3} \approx 856 \mathrm{Gpc}^{3}
$$

Within this volume, we can express the co-moving distance

$$
D_{\mathrm{c}}=\left(\frac{3 V_{\max } q}{4 \pi}\right)^{\frac{1}{3}}
$$

\footnotetext{
${ }^{1}$ http://www.astro.ucla.edu/ wright/CosmoCalc.html
} 


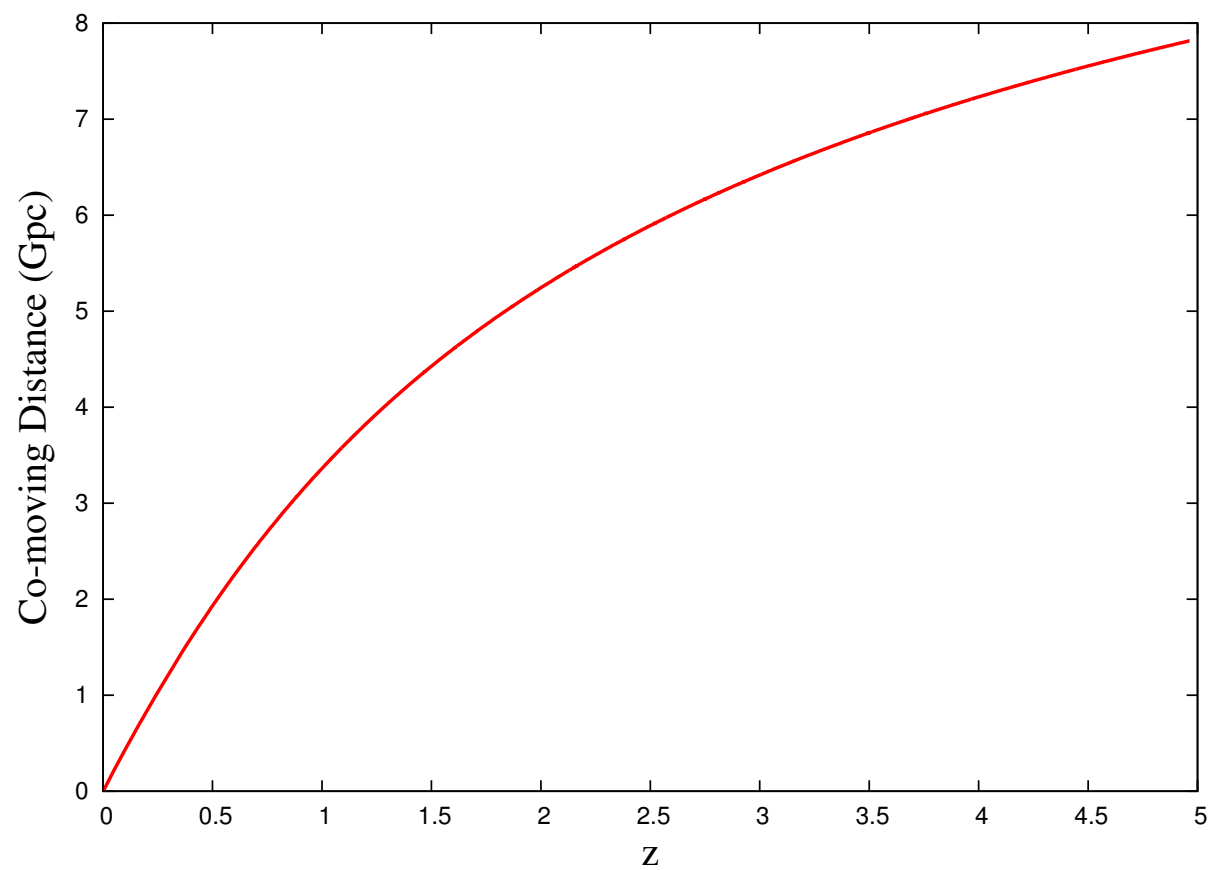

Figure 5.2: Co-moving distance as a function of redshift. The redshift is computed by numerical integration of Equation 5.7 assuming $\Omega_{\Lambda}=0.68$ and $\Omega_{m}=0.32$.

where $q$ is a random deviate ranging between 0 and 1 . Using the $\operatorname{ran}(2)$ routine of Numerical Recipes (Press et al., 1992), we compute $D_{\text {c }}$ for each FRB. Following Hogg (1999), the co-moving distance is

$$
D_{c}(z)=\frac{c}{H_{0}} \int_{0}^{z} \frac{d z^{\prime}}{\sqrt{\Omega_{m}\left(1+z^{\prime}\right)^{3}+\Omega_{\Lambda}}},
$$

where $c$ is the speed of light and the dimensionless parameters $\Omega_{m}$ and $\Omega_{\Lambda}$ have the above specified values. The co-moving distance as a function of redshift is plotted in Figure 5.2. We use this equation to generate redshifts from the co-moving distances obtained from Equation 5.6 for each of these events assuming constant co-moving number density of FRBs. We simulate 1000 FRBs for each realization. 


\subsection{DM contributions}

Once the redshift is determined, we compute the various DM contributions for each FRB. As discussed in $\S 1.4$, the DM is given by Equation 1.5. If FRBs are cosmological sources, originating in some host galaxy at a redshift of $z$ then the radiation emitted by each one is dispersed as it travels from the host galaxy to the Earth. This dispersion is a function of redshift and different dispersive regions will contribute to the total DM observed at the telescope

$$
\mathrm{DM}_{\text {observed }}=\mathrm{DM}_{\mathrm{MW}}+\mathrm{DM}_{\mathrm{IGM}}+\mathrm{DM}_{\text {host }}
$$

where $\mathrm{DM}_{\mathrm{MW}}$ is the dispersion measure within Milky Way arising due to the inter-

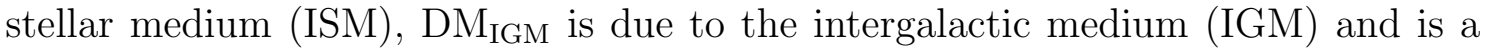
function of redshift, and $\mathrm{DM}_{\text {host }}$ is the $\mathrm{DM}$ due to the material in the host galaxy of the source.

\subsubsection{Galactic contribution}

The DM contribution arising due to the ISM in our Galaxy can be determined by integrating the NE2001 model (Cordes \& Lazio, 2002) to the edge of the Galaxy along the line of sight of the source. This model is based on the observed DMs of Galactic radio pulsars and includes contributions from the thin disk associated with low-latitude HII regions, the thick-disk, the spiral arms, small-scale features corresponding to local ISM, and individual high-density clumps and voids. We 
generate a list of $\mathrm{DM}_{\mathrm{MW}}$ values for random lines of sight with $|b|>20^{\circ}$ by integrating this model to the edge of our Galaxy. In our simulations, we choose $\mathrm{DM}_{\mathrm{MW}}$ randomly from this list for each FRB. The reason for selecting FRBs at $|b|>20^{\circ}$ is that the known FRBs in our sample (See, Table 5.1) have Galactic latitudes greater than $20^{\circ}$

\subsubsection{IGM contribution}

In this case, dispersion takes place at a range of redshifts and is an integrated effect between the source and observer. We assume that all baryons are fully ionized and homogeneously distributed so that the electron density in the IGM

$$
n_{\mathrm{e}, \mathrm{IGM}}=x(z) n_{\mathrm{e}, 0}(1+z)^{3},
$$

where $x(z)$ is the ionization fraction and $n_{\mathrm{e}, 0} \approx 2 \times 10^{-7} \mathrm{~cm}^{-3}$ is the mean local inter-galactic electron density. This gives

$$
\mathrm{DM}_{\mathrm{IGM}}=\int_{0}^{L} n_{\mathrm{e}, \mathrm{IGM}} d l
$$

Following Zheng et al. (2014), and derived in full in Appendix B, we find

$$
\mathrm{DM}_{\mathrm{IGM}}=\frac{c}{H_{0}} n_{\mathrm{e}, 0} \int_{0}^{z} d z \frac{(1+z) x(z)}{\sqrt{\Omega_{m}(1+z)^{3}+\Omega_{\Lambda}}}
$$


At low redshifts, $x(z) \simeq 0.88$ (Zheng et al., 2014). This relation is approximated by

$$
\mathrm{DM}_{\mathrm{IGM}} \simeq 1000 z
$$

which we use to compute the IGM contribution. The accuracy of this approximation is $<6 \%$ up to $z=2.5$. The use of this simplified relationship is reasonable, especially given our overall limited knowledge about the IGM structure along individual lines of sight.

\subsubsection{Host Galaxy contribution}

The only FRB for which a host galaxy has been identified unambiguously so far is a dwarf galaxy (Tendulkar et al., 2017). But it is still unclear whether the whole population of FRBs are more commonly hosted by dwarf galaxies. So, the potential host galaxy can be of any type. Since we have least information about the properties of the host galaxy, we assume that it also has similar free electron density distribution as in the Milky Way. The host contribution to DM will depend on the type of galaxy, where the source is located within it and also on the inclination angle. We generate a list of random FRB locations to account for these factors and integrate the NE2001 model to the edge of the galaxy (similar to the Milky Way) corresponding to the galactic coordinates for each of these locations. In our simulations, we choose $\mathrm{DM}_{\text {host }}$ randomly from this list. 


\subsection{Contributions to FRB widths}

In a simple empirical emission model, we set the pulse width in the rest frame

$$
W_{\text {rest }}=\frac{E}{L}
$$

where $E$ and $L$ represent the intrinsic energy and luminosity of an FRB in the rest frame. In our simulations, these are drawn from the distributions as explained in $\S 5.7 .1$. The scattering within the host galaxy will cause this rest-frame pulse to get broadened. Therefore, the time delayed intrinsic pulse width including scattering in the host galaxy is then

$$
W_{\mathrm{int}}=(1+z) \sqrt{\left(\frac{E}{L}\right)^{2}+t_{\mathrm{scatt}, \mathrm{host}}^{2}}
$$

Taking into account a number of contributing components, the effective observed pulse width of an FRB at the telescope can be expressed as

$$
W_{\mathrm{obs}}=\sqrt{W_{\mathrm{int}}^{2}+\Delta t^{2}+t_{\mathrm{scatt}, \mathrm{MW}}^{2}+t_{\mathrm{samp}}^{2}}
$$

where $\Delta t$ is the dispersive broadening across individual frequency channels due to the total DM, $t_{\text {scatt,Mw }}$ is the pulse broadening due to scattering within the Milky Way, and $t_{\text {samp }}$ is the data sampling interval for a particular survey. 


\subsubsection{Dispersive delay}

The total dispersive delay due to the total measured DM measured at the telescope across a frequency channel of width $\Delta \nu$, as explained in Chapter 1, is

$$
\Delta t \cong 8.3 \times 10^{6} \mathrm{~ms} \times \mathrm{DM} \times \frac{\Delta \nu}{\nu^{3}} .
$$

In this expression, $\Delta \nu$ and $\nu$ are in MHz. This delay causes the pulse to be smeared across the frequency channels.

\subsubsection{Scattering}

The radio waves emitted at the source are scattered by the turbulent ionized material along the line of sight which broadens the burst. This causes the radio waves to take slightly longer and variable paths to the observer. The time delay due to scattering in the interstellar medium is computed using the empirical fit from Bhat et al. (2004) and scaled to $1400 \mathrm{MHz}$ as done in Lorimer et al. (2006),

$$
\tau=0.154 \mathcal{D}+1.07 \mathcal{D}^{2}-7,
$$

where $\tau$ and $\mathcal{D}$ are the base-10 logarithms of $t_{\text {scatt }}$ (in ms) and DM (in $\mathrm{cm}^{-3} \mathrm{pc}$ ) respectively. The same scattering model is used for the host galaxy. For both scattering cases, we compute the scattering times using a Gaussian distribution centered at $\tau$ with a standard deviation of 0.8 .

It seems highly unlikely that the IGM follows the same scattering relationship 
as in the Milky Way. Also, Cordes et al. (2016) argue that the non-detection of FRBs in directions through the inner Galaxy is due to large Galactic scattering, causing the pulse to get smeared out and therefore host galaxies dominate pulse broadening. For the known FRBs, the scattering due to the Milky Way is of the order of a few $\mu$ s. If the IGM contributes to scattering then that would require a level of turbulence an order of magnitude higher than encountered at the Galactic center (Macquart \& Koay, 2013). Low density IGM cannot support these density fluctuations. Accounting for these arguments and lack of reliable IGM scattering model, we assume that the contribution to scattering from IGM is negligible in our simulations.

\subsection{Peak fluxes of FRBs}

To compute the peak flux at a redshift $z$ measured over a certain bandwidth $\nu_{2}-\nu_{1}$ (see Table 5.2), we use Equation 4 of Lorimer et al. (2013) which gives the energy

$$
E_{\nu^{\prime}}=k \nu^{\prime \alpha}
$$

where $k$ is a constant, $\nu^{\prime}$ is the rest-frame frequency and $\alpha$ is a spectral index. In the rest frame of the source, from conservation of energy, for an observation over some frequency band between $\nu_{1}$ and $\nu_{2}$, we get Equation 9 of Lorimer et al. (2013),

$$
S_{\text {peak }}=\frac{L(1+z)^{\alpha-1}}{4 \pi D(z)^{2}\left(\nu_{2}^{\prime \alpha+1}-\nu_{1}^{\prime \alpha+1}\right)}\left(\frac{\nu_{2}^{\alpha+1}-\nu_{1}^{\alpha+1}}{\nu_{2}-\nu_{1}}\right) .
$$


For an FRB at a redshift $z$ with a bolometric luminosity $L$ and observed pulse width $W_{\text {obs }}$, the peak flux is

$$
S_{\text {peak }}=\frac{L(1+z)^{\alpha-1}}{4 \pi D(z)^{2}\left(\nu_{2}^{\prime \alpha+1}-\nu_{1}^{\prime \alpha+1}\right)}\left(\frac{\nu_{2}^{\alpha+1}-\nu_{1}^{\alpha+1}}{\nu_{2}-\nu_{1}}\right) \frac{W_{\mathrm{int}}}{W_{\mathrm{obs}}} .
$$

Although we record radio signals across a small observing band at the telescope, the radio emission at the source covers a broader range of frequencies. We adopt $\nu_{2}^{\prime}=10 \mathrm{GHz}, \nu_{1}^{\prime}=10 \mathrm{MHz}$, and assume a spectral index $\alpha=-1.4$, consistent with the radio pulsar population. The observed flux $S_{\text {peak,obs }}$ is in practice less than $S_{\text {peak }}$ due to the finite size of the telescope beam. To calculate $S_{\text {peak,obs }}$, we adopt a Gaussian beam, so that

$$
S_{\text {peak }, \text { obs }}=S_{\text {peak }} \exp \left[-\frac{r^{\prime 2}}{2 \ln 2 r_{\text {beam }}^{2}}\right]
$$

where $r^{\prime}$ is the distance from the beam center chosen randomly for each simulated FRB and $r_{\text {beam }}$ is the beam radius.

The 13 beams of the Parkes multibeam receiver have different gains and slightly different beam radii. The telescope gain $G$ quantifies the radio noise power received from a source of unit flux density and is expressed in units of $\mathrm{K} \mathrm{Jy}^{-1}$ (Staveley-Smith et al., 1996; Manchester et al., 2001). For beam 1, the beam radius is $7.0^{\prime}$ and at the center of this beam, the gain is $0.731 \mathrm{~K} \mathrm{Jy}^{-1}$. Beams $2-7$ represent the inner ring of the multibeam receiver each with beam radius of $7.05^{\prime}$ and the gain corresponding to each beam center is $0.690 \mathrm{~K} \mathrm{Jy}^{-1}$. Beams $8-13$ represent the outer ring of the 
multibeam receiver each with beam radius of $7.25^{\prime}$ and the gain corresponding to each beam center is $0.581 \mathrm{~K} \mathrm{Jy}^{-1}$. For a more realistic approach, we randomly select both a beam number for each FRB and a distance from the beam center $r^{\prime}$. Then using the corresponding gain for that beam and $r^{\prime}$, we compute $S_{\text {peak,obs }}$ for each simulated FRB.

\subsection{Signal-to-noise ratio}

The single-pulse search algorithm searches for pulses of different widths by adding adjacent samples in the de-dispersed time series. The pulse is detected for a trial width $W_{\text {trial }}$ closest to the observed effective width measured at the telescope $W_{\text {obs. }}$ The $\mathrm{S} / \mathrm{N}$ for optimal detection is then

$$
S / N=\frac{S_{\text {peak }, \text { obs }}}{\sigma_{\text {rms }}}
$$

where

$$
\sigma_{\mathrm{rms}}=\frac{\beta T_{\mathrm{sys}}}{G \sqrt{2 \Delta \nu W_{\text {trial }}}}
$$

Here $\beta$ is the digitization factor (Kouwenhoven \& Voûte, 2001), and $T_{\text {sys }}$ is the system temperature. We simulate FRBs using the survey parameters of the HTRU high latitude survey. This survey is chosen because half of the currently published population was discovered in this survey. The survey parameters are listed in Table 5.2 .

Ideally, if the trial width matches exactly with the observed width then the 
Table 5.2: System parameters for Parkes HTRU high latitude survey obtained from Thornton (2013).

\begin{tabular}{lc}
\hline Parameter & Value \\
\hline$T_{\text {sys }}$ & $30 \mathrm{~K}$ \\
$\Delta \nu$ & $340 \mathrm{MHz}$ \\
$\Delta \nu_{\mathrm{ch}}$ & $0.39 \mathrm{MHz}$ \\
$\beta$ & 1.07 \\
$t_{\text {samp }}$ & $64 \mu \mathrm{s}$ \\
\hline
\end{tabular}

observed peak flux is the optimal flux. But we do not try every pulse width. For a more realistic approach, in our simulations as done for the single-pulse searching in the HTRU survey of the Parkes telescope (Thornton, 2013), we generate trial widths in powers of two and find a closest effective width to one of the trial widths for each simulated FRB. The trial widths considered in our analysis are 0.064, 0.128, 0.256, $0.512,1.024,2.048,4.096,8.192,16.384,32.768 \mathrm{~ms}$, same as chosen in the processing of the HTRU survey (Thornton, 2013). If $W_{\text {trial }}<W_{\text {obs }}$, then the observed peak flux is lowered by a factor $W_{\text {trial }} / W_{\text {obs }}$. In this case, the $\mathrm{S} / \mathrm{N}$ can be computed from

$$
S / N=\frac{\left(W_{\text {trial }} / W_{\text {obs }}\right) S_{\text {peak }, \mathrm{obs}}}{\sigma_{\mathrm{rms}}}=\frac{S_{\text {peak }, \mathrm{obs}} G \sqrt{2 \Delta \nu}}{\beta T_{\mathrm{sys}}} \frac{W_{\text {trial }}^{1.5}}{W_{\mathrm{obs}}}
$$

If the trial width is greater than the observed effective width then the observed peak flux is reduced by a factor $W_{\text {obs }} / W_{\text {trial }}$ and the measured $\mathrm{S} / \mathrm{N}$ can be computed from

$$
S / N=\frac{\left(W_{\text {obs }} / W_{\text {trial }}\right) S_{\text {peak }, \text { obs }}}{\sigma_{\text {rms }}}=\frac{S_{\text {peak }, \text { obs }} G \sqrt{2 \Delta \nu}}{\beta T_{\text {sys }}} \frac{W_{\text {obs }}}{\sqrt{W_{\text {trial }}}} .
$$




\subsection{Simulation procedure}

For a particular model based on the luminosity $L$ and energy $E$ distributions described below, we carry out the simulations until we reach a sample of 1000 detectable FRBs for each realization. For each simulated FRB, $E$ and $L$ are chosen based on a particular model and redshift, DM, observed pulse width, peak observed flux, and $\mathrm{S} / \mathrm{N}$ are computed following the steps summarized in the flowchart (Figure 5.3). Once the $\mathrm{S} / \mathrm{N}$ is obtained, a detection criteria is applied. We set a minimum $\mathrm{S} / \mathrm{N}$ of nine for the FRB to be detected in the HTRU survey (as done in the processing of this survey, Thornton 2013). The three models explored in this analysis are referred to as models A, B, and C. In model A, we use zero mean Gaussians for $L$ and $E$. The standard deviations of these distributions are $\sigma_{\mathrm{E}}$ and $\sigma_{\mathrm{L}}$. For each simulated FRB, only positive values of energy and luminosity are chosen from these distributions. Initially, we tried an even simpler model with constant $E$ and $L$ values. However, the simulated population did not quite cover the observed population. Model A was an attempt to improve upon this by assigning a range to the $L$ and $E$ values. The zero mean Gaussian models reflect the idea that we might expect more faint sources than bright ones.

In model B, we use a power law luminosity distribution and a constant energy for an FRB. For each simulated FRB, a luminosity is drawn from this power law model with some minimum luminosity $L_{\min }$ and a power law index $\alpha_{\mathrm{L}}$ as described in Section 5.1. The energy for each FRB is constant, drawn from an energy range.

In model C, we use a power law luminosity distribution and zero mean Gaus- 


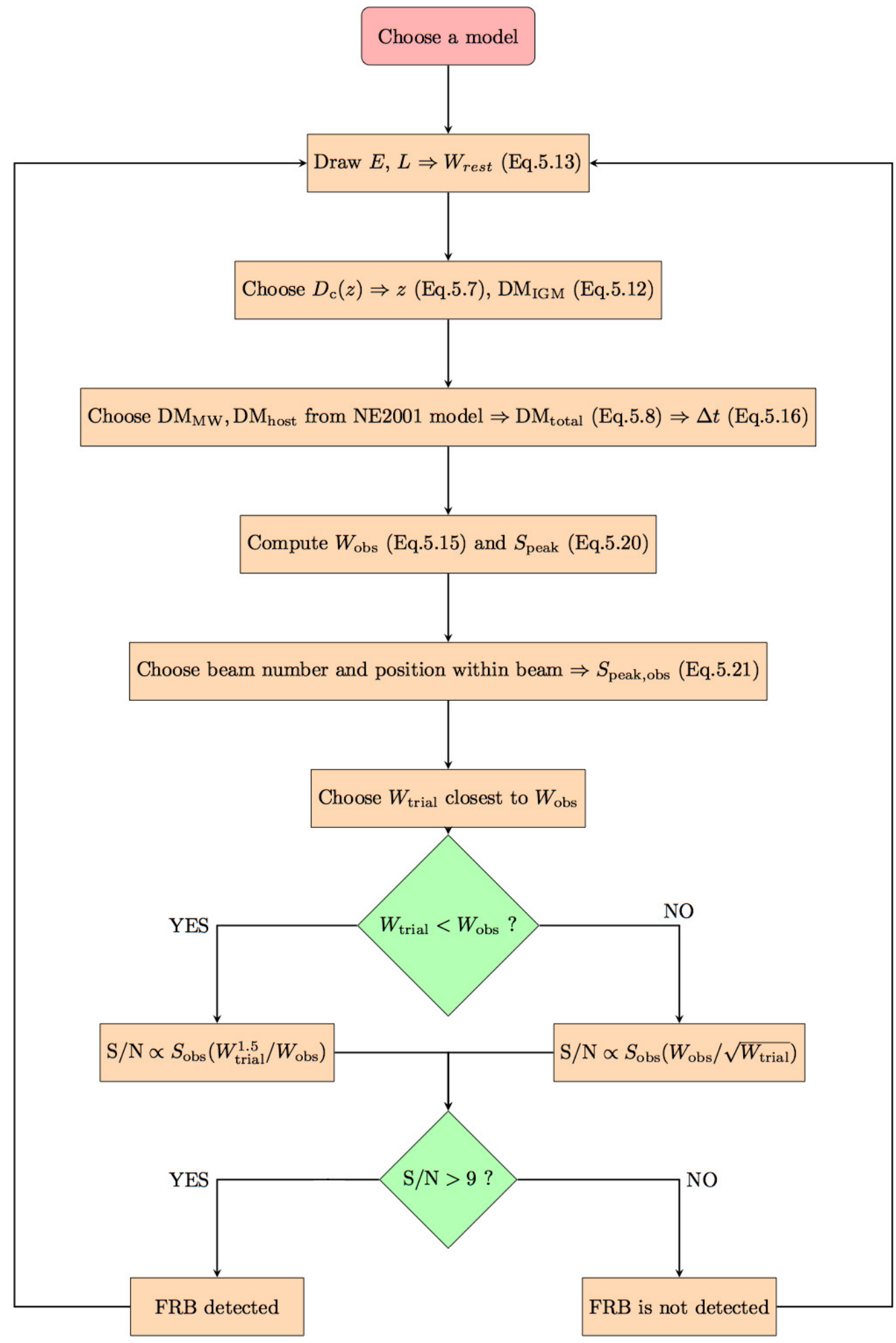

Figure 5.3: A flowchart describing the steps involved in the simulations. 
sian for $E$. For each simulated FRB, a luminosity is drawn in the same way as in Model B and energy is drawn in the same way as in Model A.

As mentioned earlier, it is very likely that host galaxies dominate pulse broadening. In order to implement this scenario in our simulations, we explore two cases for each model listed above. Case 1 refers to scattering arising only in the host galaxy. The models in this case are labeled as A1, B1, and C1. Case 2 refers to significant scattering both in the MW and from the host galaxy. The models in this case are labeled as A2, B2, and C2. Both of these scenarios use Equation 5.17 to compute scattering in the Milky Way and in the host galaxy.

We find the minimum and maximum of the DMs, peak fluxes, and observed widths for the observed sample considering $10 \%$ uncertainties in the same. For each observed FRB, we find a cell with the above mentioned uncertainties in DMs, fluxes, and effective widths and count the number of modeled FRBs in each cell.

The next step is to compute the probability of getting the modeled number of FRBs in each cell. For the $i^{t h}$ cell, this probability is given by

$$
p_{\mathrm{i}}=\frac{N_{\mathrm{i}}}{N_{\mathrm{sim}}},
$$

where $N_{\mathrm{i}}$ is the number of modeled FRBs in cell $i$ and $N_{\mathrm{sim}}=1000$ is the total number of detectable FRBs simulated for a model. If $N_{\mathrm{i}}$ is zero then the predicted probability is set to $1 / 1000$ instead (i.e., an upper limit). The likelihood function 
for one iteration of a model is

$$
\mathcal{L}\left(\vec{x} \mid D M, S_{\text {peak }, \text { obs }}, W_{\text {obs }}\right)=\prod_{i=1}^{n} p_{\mathrm{i}}
$$

and is described by a set of parameters $\vec{x}$, and observed values of DM, $S_{\text {peak,obs }}, W_{\text {obs }}$ of known FRBs. Here $n$ is the number of known FRBs $(n=14$, see Table 5.1). For model $\mathrm{A}, \vec{x}=\left[\sigma_{\mathrm{E}}, \sigma_{\mathrm{L}}\right]$. For model $\mathrm{B}, \vec{x}=\left[E, L_{\mathrm{min}}, \alpha_{\mathrm{L}}\right]$. And for model $\mathrm{C}$, $\vec{x}=\left[\sigma_{\mathrm{E}}, L_{\min }, \alpha_{\mathrm{L}}\right]$

\subsection{Maximum likelihood analysis}

We compute the likelihood as described in previous section over a wide range of energy and luminosity values. We select energy values in the range $10^{38}-10^{44}$ ergs and luminosity values in the range $10^{39}-10^{46} \operatorname{ergs~s}^{-1}$ and determine $E$ and $L$ values with the highest likelihood within this range (see Table 5.3). This brute-force method is quite time consuming and since each of our models have two or more free parameters, much of the parameter space does not contribute to the region of higher likelihood values. This is why we use the more efficient Markov Chain Monte Carlo (MCMC) method to explore the parameter space for each of our models.

We compute the likelihoods for each model using the Metropolis-Hastings algorithm (Metropolis et al., 1953; Hastings 1970). The steps followed are

1. To initiate a Markov chain, start with the initial set of parameters $\vec{x}$ for each model determined from the brute-force method (see Table 5.3). So we have 
Table 5.3: The initial set of parameters for each model.

\begin{tabular}{|c|c|c|c|}
\hline Model & \multicolumn{3}{|c|}{ Parameter values } \\
\hline & $\sigma_{\mathrm{E}}\left(\times 10^{42} \mathrm{ergs}\right)$ & $\sigma_{\mathrm{L}}\left(\times 10^{45} \mathrm{ergs} \mathrm{s}^{-1}\right)$ & \\
\hline A1 & 2.03 & 1.03 & \\
\hline A2 & 2.10 & 1.11 & \\
\hline & $E\left(\times 10^{41} \mathrm{ergs}\right)$ & $L_{\min }\left(\times 10^{44} \operatorname{ergs~s}^{-1}\right)$ & $\alpha_{L}$ \\
\hline B1 & 0.23 & 4.12 & 2.17 \\
\hline B2 & 0.28 & 4.35 & 2.00 \\
\hline & $\sigma_{\mathrm{E}}\left(\times 10^{41} \mathrm{ergs}\right)$ & $L_{\min }\left(\times 10^{44} \mathrm{ergs} \mathrm{s}^{-1}\right)$ & $\alpha_{L}$ \\
\hline $\mathrm{C} 1$ & 1.43 & 5.51 & 2.54 \\
\hline $\mathrm{C} 2$ & 0.86 & 4.60 & 1.87 \\
\hline
\end{tabular}

$\vec{x}_{1}=\vec{x}$. Compute the likelihood for this set of parameters, $\mathcal{L}_{1}$.

2. Choose $\vec{x}_{2}$ from a Gaussian distribution centered at $\vec{x}_{1}$ with a standard deviation of $0.1 \vec{x}_{1}$ such that

$$
f\left(\vec{x}_{2}\right)=\exp \left[\frac{-\left(\vec{x}_{2}-\vec{x}_{1}\right)^{2}}{2\left(0.1 \vec{x}_{1}\right)^{2}}\right] .
$$

For this set of parameters, compute $\mathcal{L}_{2}$.

3. Compute the Metropolis ratio: $s=\mathcal{L}_{2} / \mathcal{L}_{1}$.

4. Choose a random deviate $q$ from a uniform distribution in the range $0 \leq q \leq 1$.

5. If $q>s$, then stay at the current position. If $q<s$, then move to the new position.

6. Repeat this process by choosing a new candidate position and random deviate until the chain converges.

The same procedure is followed for the three models, each for the two scattering 
cases. In each Markov chain, we carried out 6000 iterations.

\subsection{Results}

We now discuss the results of the MCMC method for one of the models here. The left panels of Figure 5.4 are the so-called traceplots showing the running mean of each parameter over the entire 6000 iterations of the MCMC for model C2. Traceplots for the other models are given in Appendix 3. In a Markov chain, the initial samples generated starting with the initial values do not describe the observed population very well producing very low likelihoods. However, the MCMC algorithm generates samples from the model parameter space such that the probability for being in that region is proportional to the likelihood. The samples are not independently chosen from their parameter spaces, instead, the new sample depends on the previous sample and hence the free parameters are correlated. The initial set of steps in the chain that do not accurately sample the range of high likelihood values is

referred to as the burn-in period seen in Figure 5.4. This period is corresponding to first 2000 iterations for this particular model (region before the dashed vertical line). The sampled values of the free parameters fluctuate over a wide range, whereas, after the burn-in period, the free parameters are sampled over a broad range, eventually converging to a stationary distribution giving maximum likelihood. The marginalized PDFs for each parameter after satisfactory convergence are shown in the right panels and are constructed after discarding the first 2000 iterations.

The maximum likelihood values and the $95 \%$ confidence intervals for all models 

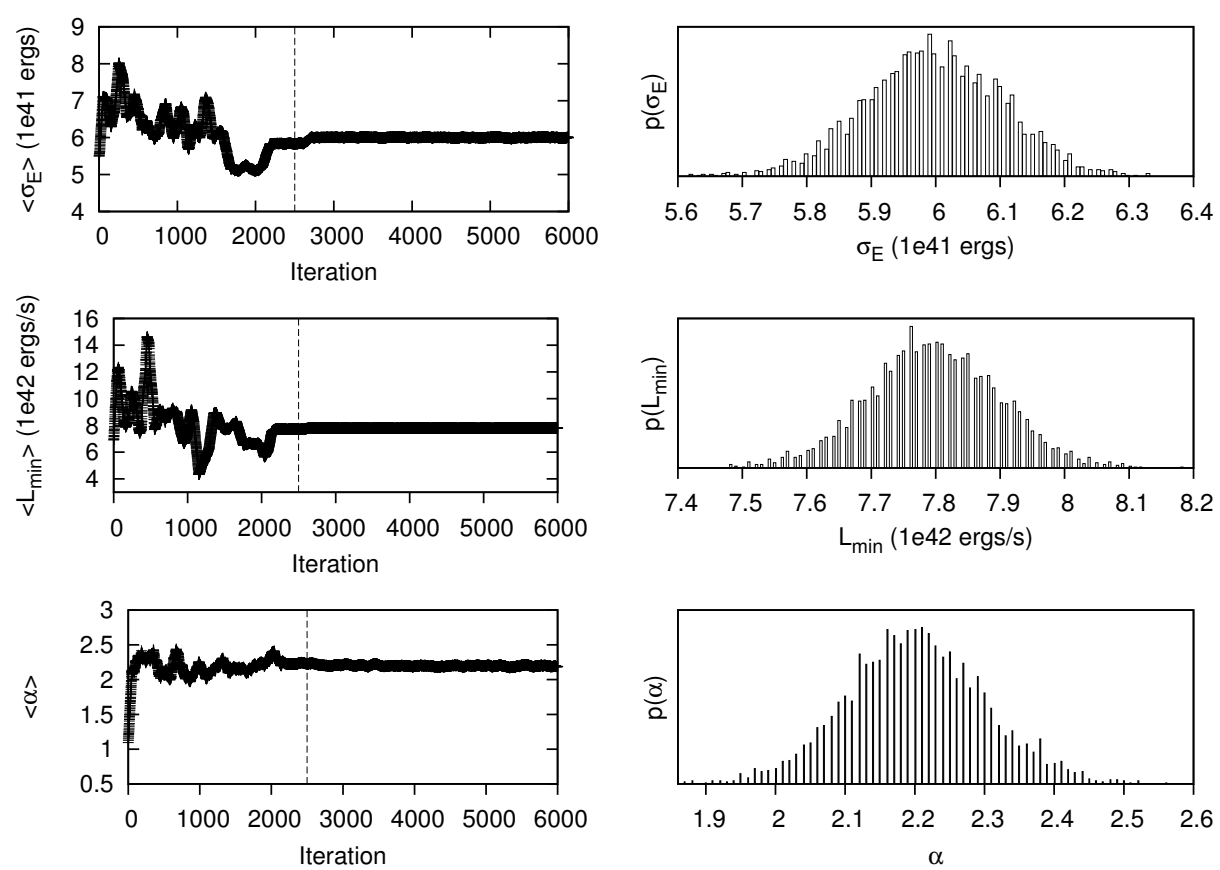

Figure 5.4: MCMC results for model $\mathrm{C} 2$ with scattering in the Milky Way and from host galaxy. The left panel are traceplots showing the running mean of each parameter over the entire 6000 iterations of the MCMC. The region before the dashed vertical line represents the burn-in period. The marginalized PDFs for each parameter are constructed after discarding the steps before this burn-in period and are shown in the right panels. 
Table 5.4: The optimal parameters and the maximum likelihood for each model

\begin{tabular}{|c|c|c|c|c|}
\hline Model & \multicolumn{3}{|c|}{ Optimal parameters } & $\log _{10}(\mathcal{L})$ \\
\hline & $\sigma_{\mathrm{E}}\left(\times 10^{41} \mathrm{ergs}\right)$ & $\sigma_{\mathrm{L}}\left(\times 10^{44} \operatorname{ergs~s}^{-1}\right)$ & & \\
\hline A1 & $14.5_{-0.3}^{+0.4}$ & $5.2_{-0.3}^{+0.3}$ & & -20.1 \\
\hline $\mathrm{A} 2$ & $22.1_{-0.4}^{+0.3}$ & $6.3_{-0.3}^{+0.4}$ & & -20.3 \\
\hline & $E\left(\times 10^{41} \mathrm{ergs}\right)$ & $L_{\min }\left(\times 10^{42} \operatorname{ergs~s}^{-1}\right)$ & $\alpha_{L}$ & \\
\hline B1 & $1.3_{-0.3}^{+0.4}$ & $1.8_{-0.3}^{+0.3}$ & $1.9_{-0.3}^{+0.3}$ & -18.8 \\
\hline $\mathrm{B} 2$ & $1.7_{-0.3}^{+0.3}$ & $1.8_{-0.4}^{+0.4}$ & $1.9_{-0.3}^{+0.3}$ & -18.9 \\
\hline & $\sigma_{\mathrm{E}}\left(\times 10^{41} \mathrm{ergs}\right)$ & $L_{\min }\left(\times 10^{42} \operatorname{ergs~s}^{-1}\right)$ & $\alpha_{L}$ & \\
\hline $\mathrm{C} 1$ & $4.0_{-0.3}^{+0.3}$ & $6.9_{-0.3}^{+0.4}$ & $2.2_{-0.3}^{+0.5}$ & -18.5 \\
\hline $\mathrm{C} 2$ & $6.0_{-0.4}^{+0.3}$ & $7.8_{-0.3}^{+0.3}$ & $2.2_{-0.3}^{+0.3}$ & -18.4 \\
\hline
\end{tabular}

are listed in Table 5.4. Out of the three models considered in our analysis, models $\mathrm{B}$ and $\mathrm{C}$ give higher likelihood values compared to model A. Both of these models represent the observed FRB population quite well and the model with a Gaussian distributed energy and a power law distribution in luminosity (model C2) gives the highest likelihood compared to the other models. We therefore explore this model further in our analysis.

\subsection{Discussion}

We have performed MCMC simulations of an FRB population with different energy and luminosity distributions. In this section, we will discuss the results of the best-fit model. Model C2, which follows a power law luminosity and a Gaussian distributed energy distribution with scattering contribution from both the Milky Way and the host galaxy, has the highest log likelihood compared to the other models considered in our analysis. The optimal value of the standard deviation in energy is $6.0 \times 10^{41} \mathrm{ergs}$. The optimal values of the power law luminosity distribution 


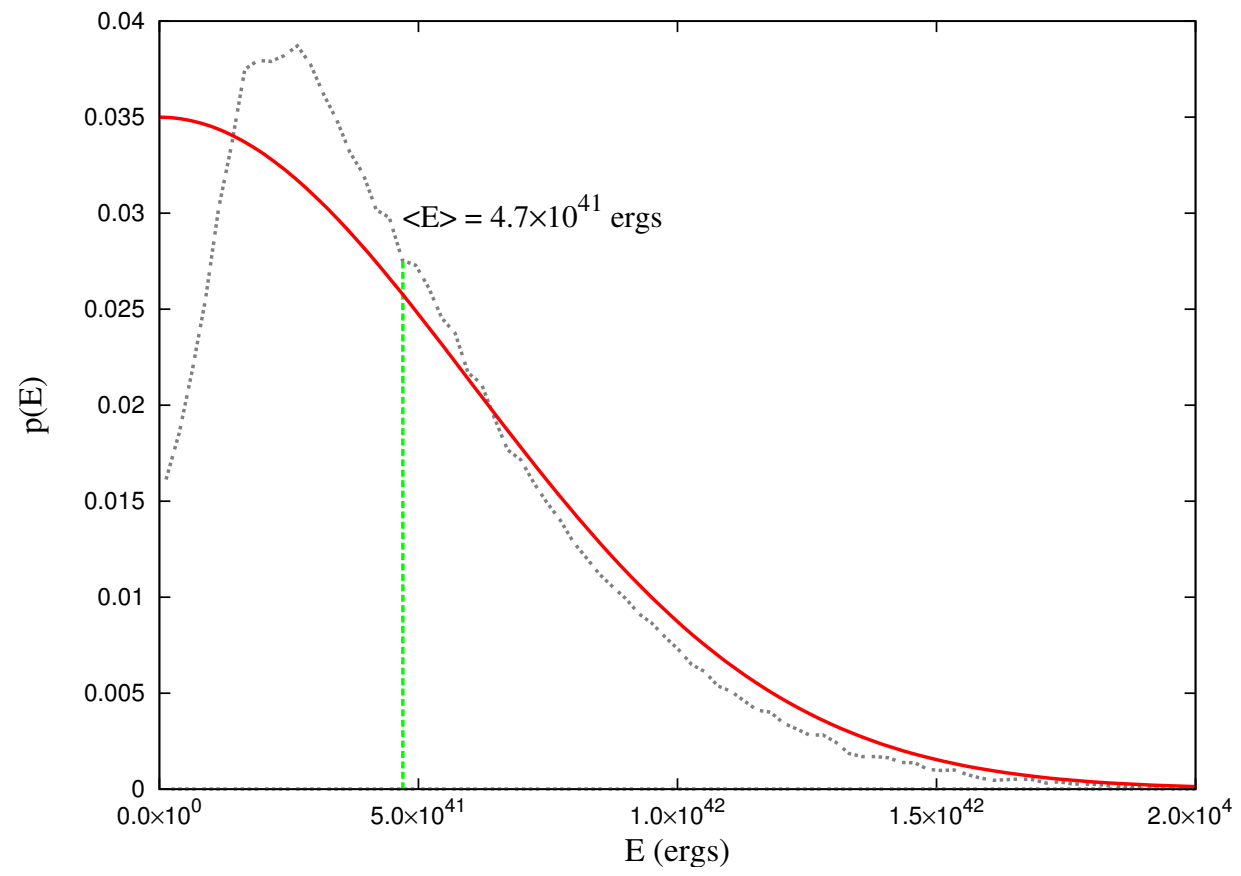

Figure 5.5: Energy distributions of the detectable modeled FRB population (dotted line) and for FRBs with optimal parameters listed in Table 5.4 (red solid line). The mean energy of the detectable population is $4.7 \times 10^{41} \mathrm{ergs}$.

has a minimum luminosity of $7.8 \times 10^{42} \mathrm{ergs} \mathrm{s}^{-1}$ with a power law index of 2.3. For this set of parameters, Figure 5.5 and Figure 5.6 show the energy and power law luminosity distributions of the detectable modeled FRB population (dotted lines). The mean energy of this distribution is $4.7 \times 10^{41} \mathrm{ergs}$ and the mean luminosity is $1.1 \times$ $10^{45} \mathrm{ergs} \mathrm{s}^{-1}$. The solid red lines are the distributions for the optimal parameters.

The observable parameters of this population are compared with the known population and Figure 5.7 shows that these two populations are consistent for this model. In this figure, the left panel compares the observed peak fluxes versus the measured DM for modeled FRBs (red dots) with the known FRBs (black diamonds). The right panel compares the observed peak fluxes versus the observed effective widths for the same two populations. The dips in this plot are due to the limited 


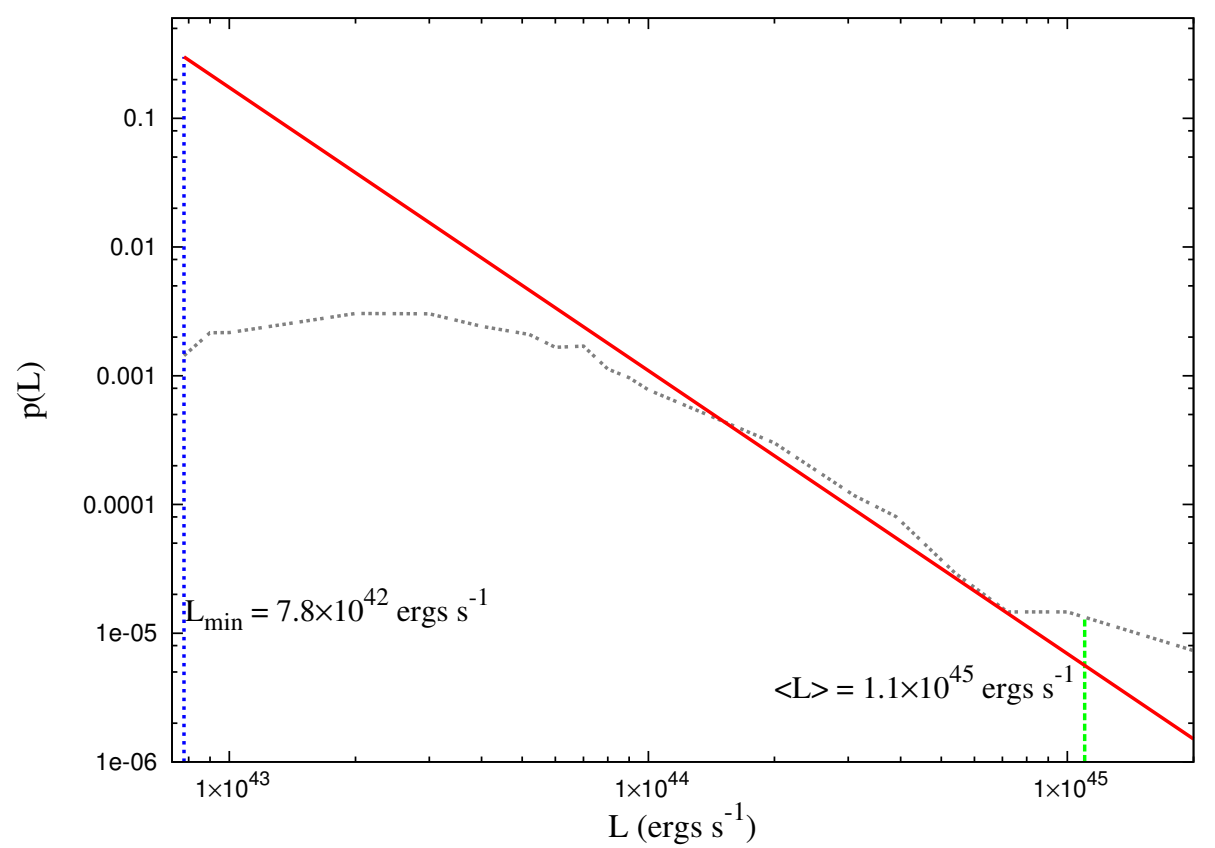

Figure 5.6: Luminosity distribution of the detectable modeled FRB population (dotted line) and for FRBs with optimal parameters listed in Table 5.4 (red solid line). The mean luminosity of the detectable population is $1.1 \times 10^{45} \mathrm{ergs} \mathrm{s}^{-1}$.

number of trial widths considered in our analysis. Also, it can be seen that no FRBs are detectable in the $\mathrm{DM}<200 \mathrm{~cm}^{-3}$ pc and $S_{\text {peak,obs }}<2$ Jy range.

To explore this region, we have plotted the fluxes and $\mathrm{S} / \mathrm{N}$ of these undetectable FRBs in Figure 5.8. All of these FRBs have S/N lower than the threshold due to low peak fluxes and larger widths (Equation 5.24), for trial widths smaller than the observed widths. In order to verify that the modeled FRBs are sampling the parameter space well, we have repeated plots in Figure 5.7 for a sample of only 20 modeled FRBs shown in Figure 5.9.

The sky distribution of modeled FRB in each sample cell (triangles) is also consistent with that of the known FRBs (circles) as shown in Figure 5.10. Both the populations are uniformly distributed with no preference along any particular lines 

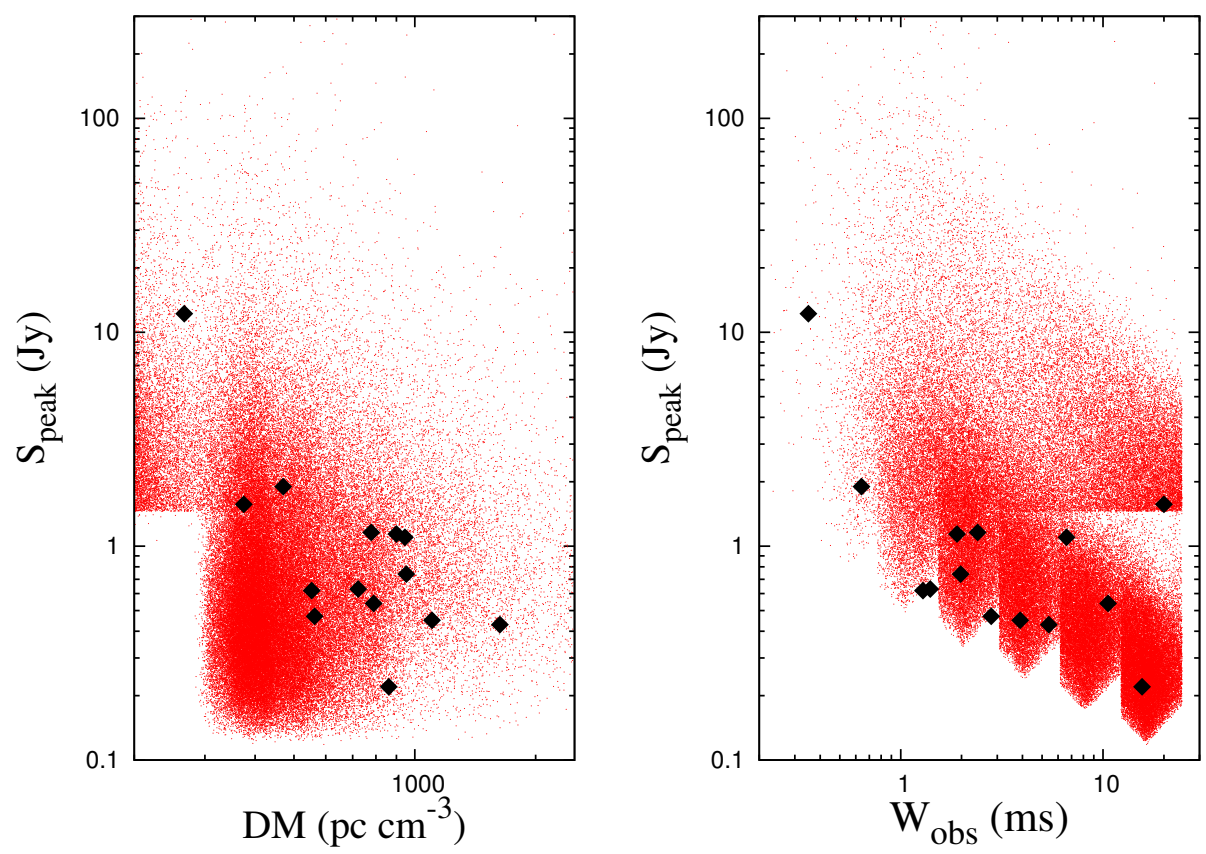

Figure 5.7: The left panel shows the observed peak flux versus the measured DM for modeled FRBs (red dots) and known FRBs (black diamonds). The right panel shows the observed peak fluxes versus the observed effective widths for the same two populations.

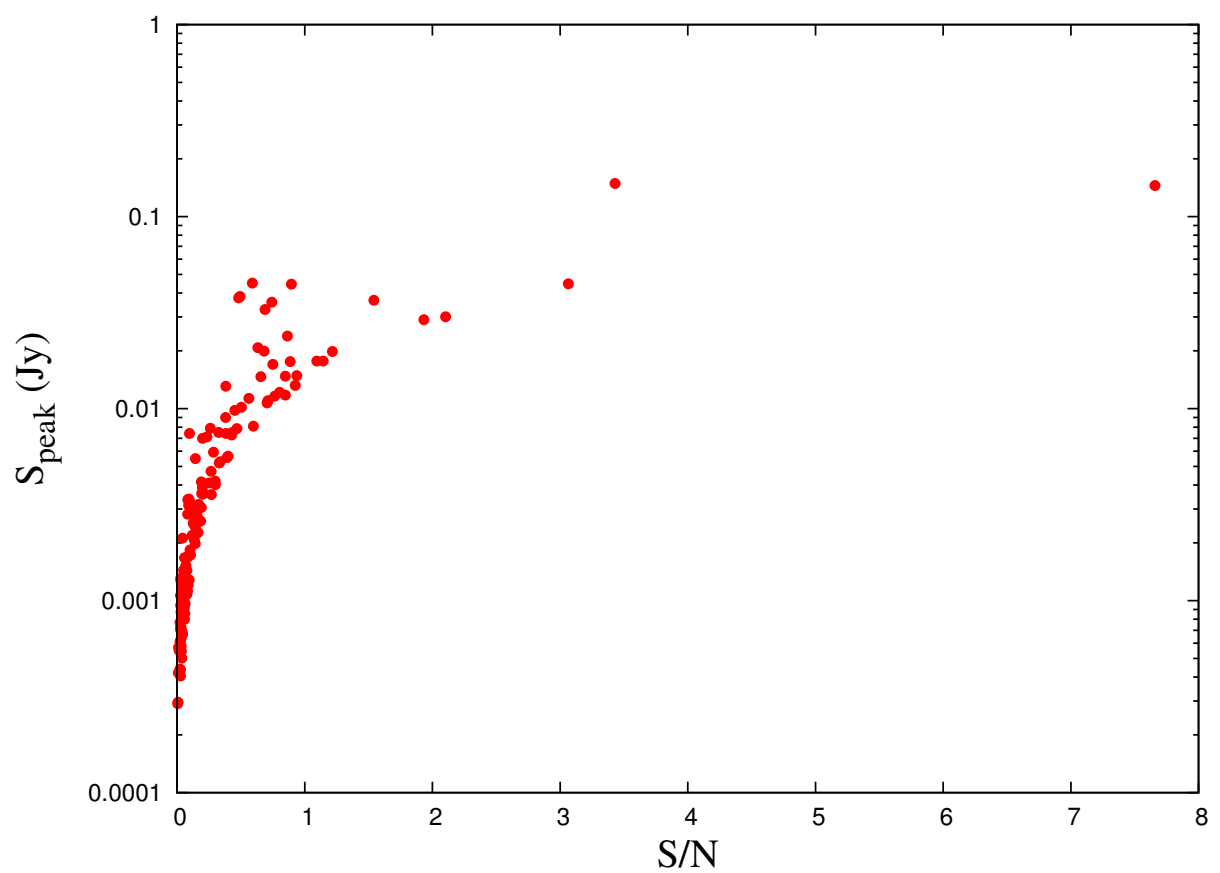

Figure 5.8: The figure shows the peak observed fluxes versus $\mathrm{S} / \mathrm{N}$ of undetectable FRBs that lie in the left bottom corner of the same plot from Figure 5.7. 

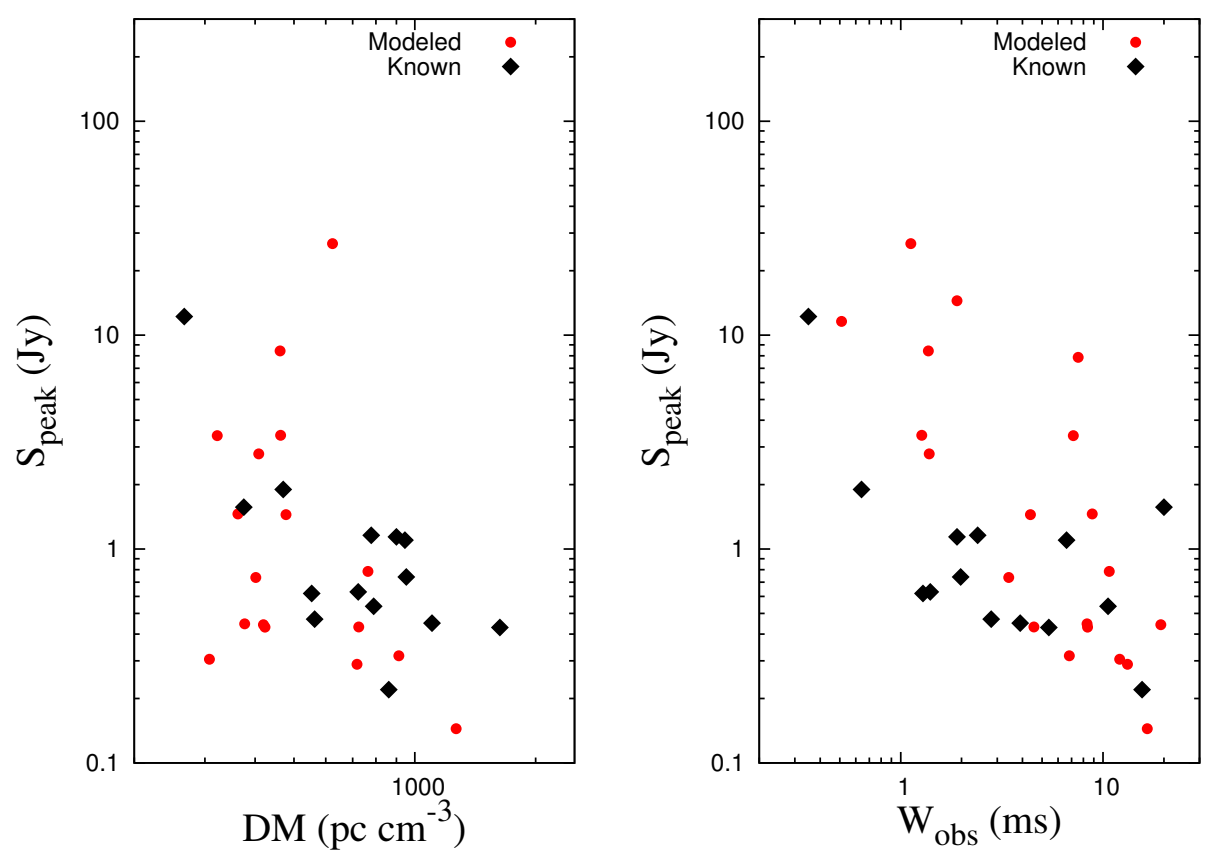

Figure 5.9: The left panel shows the observed peak flux versus the measured DM for modeled FRBs (red dots) and known FRBs diamonds. The right panel shows the observed peak fluxes versus the observed effective widths for the same two populations.

of sight. In our simulations, we generate $1000 \mathrm{FRBs}$ that are detectable with the Parkes multi-beam setup up to a redshift of 2.5. Figure 5.11 shows the fraction of detectable FRBs in a co-moving shell between $z$ and $z+d z$ as a function of redshift (and co-moving distance). More FRBs are detectable in shells at lower redshifts compared to all FRBs (detectable and non-detectable) generated in the simulation for the same co-moving shells. As we go to higher redshifts, fewer FRBs (very bright ones) out of the total simulated population are detectable as expected.

\subsubsection{The $\log N-\log S$ relation of FRB events}

The number of sources as a function of flux $(\log N-\log S)$ is an important tool for describing and investigating the properties of various types of source populations 


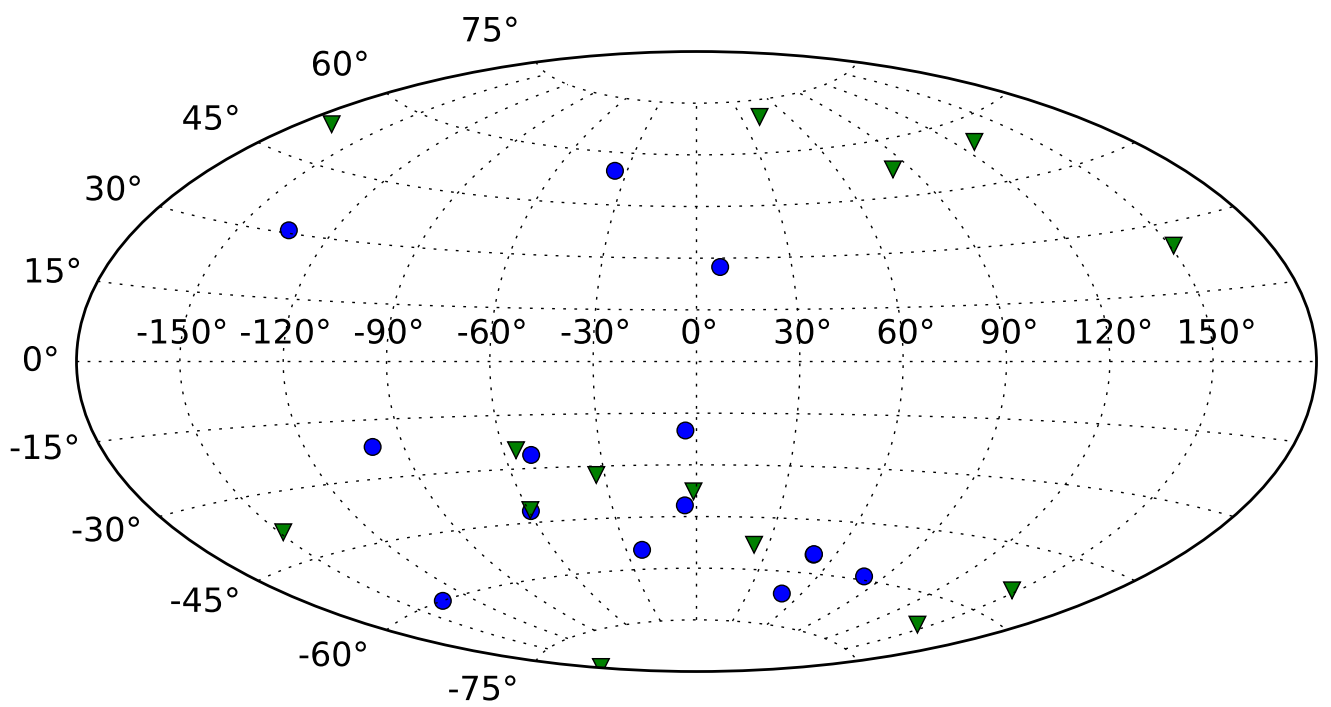

Figure 5.10: The sky distribution of modeled FRB in each sample cell (triangles) and the known FRB in the same cell (circles).

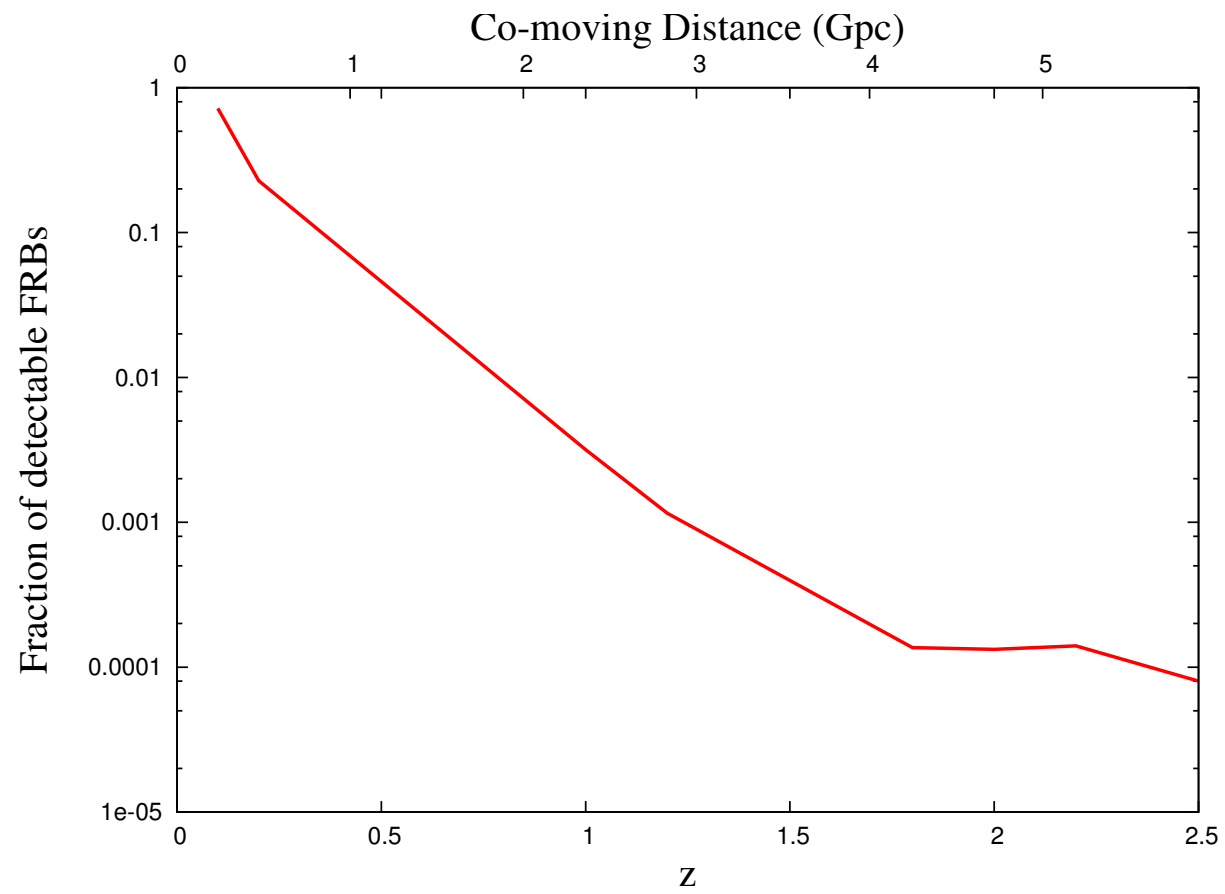

Figure 5.11: Fraction of detectable FRBs in co-moving shell between $z$ and $z+d z$ as a function of redshift and co-moving distance. 
and their distribution in space. For sources of given intrinsic luminosity distributed uniformly throughout the Euclidean universe, we expect the number of sources $N$ detected above some flux threshold $S$ to vary as $N \propto S^{\alpha}$, where $\alpha=-3 / 2$. This is shown in Figure 5.12 for sources within a redshift of 1 having a constant luminosity and the dashed line is a best-fitting line with a slope of $-3 / 2$. However, in our models $B$ and $C$, we simulate FRBs with a broad luminosity distribution up to a redshift of 2.5. This dispersion in intrinsic luminosities indicates a less steep slope. Additionally at such distances, non-Euclidean effects are important. Figure 5.13 describes the observed cumulative distribution of modeled FRBs before (dotted line) and after (solid line) flux degradation and the same for all known Parkes FRB sample (crosses). The line with a slope of -1.5 is shown for reference (gray line). These three curves have slopes of $-1.3,-1.3$, and -0.9 respectively. These values of slopes are consistent with the previous study by Caleb et al. (2016).

\subsubsection{Tracking star formation history}

The basic assumption in our analysis is that FRBs are uniformly distributed over a co-moving volume up to a maximum redshift of 2.5. However, if we find FRBs at higher redshifts (for example, $z>2$ ) then this assumption might not be valid as the rate of star formation is not constant with redshift. Most of the progenitor models suggested for repeating and non-repeating FRBs involve either cataclysmic events from collapsing massive stars or giant flares from magnetars or phenomena involving young neutron stars. These models indicate that the redshift distribution 


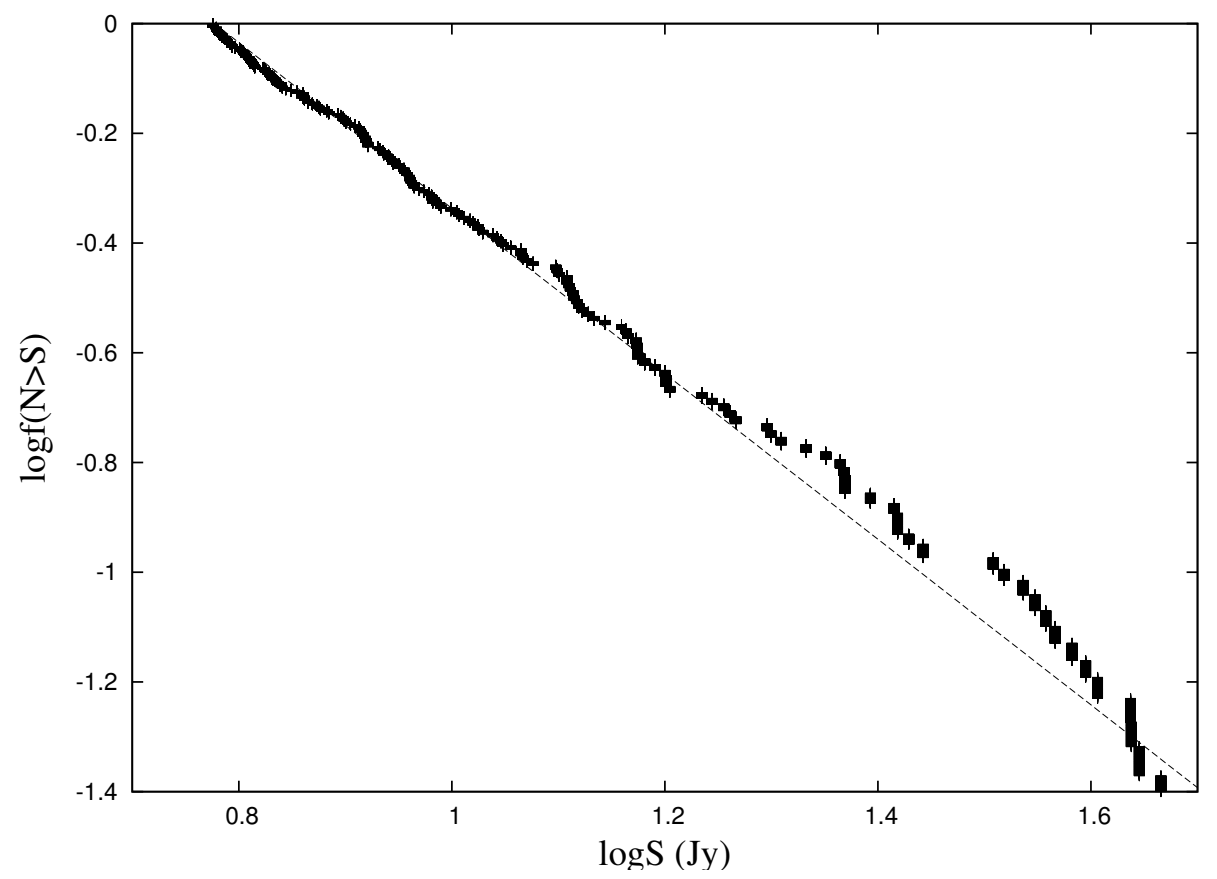

Figure 5.12: The $\log N-\log S$ relation for sources (points) with redshifts less than 1. The dashed line is a best-fitting line with a slope of $-3 / 2$.

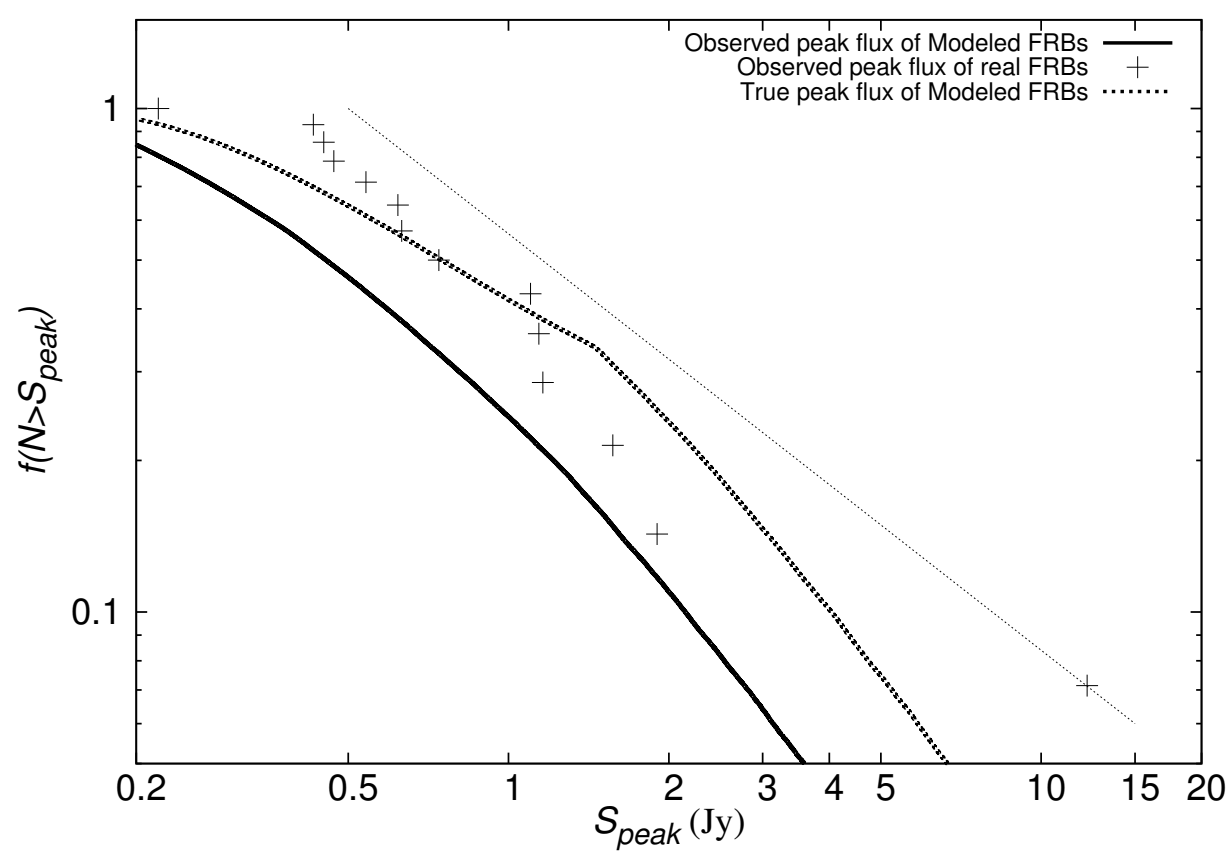

Figure 5.13: The $\log N-\log S$ curves for the Parkes FRBs and the modeled FRBs. The solid line represents the the observed cumulative distribution of modeled FRBs, whereas the dotted line represents the same of modeled FRBs before accounting for flux degradation. The crosses are the known FRBs in our sample and the line with a slope of -1.5 is shown for reference (dotted gray line). 


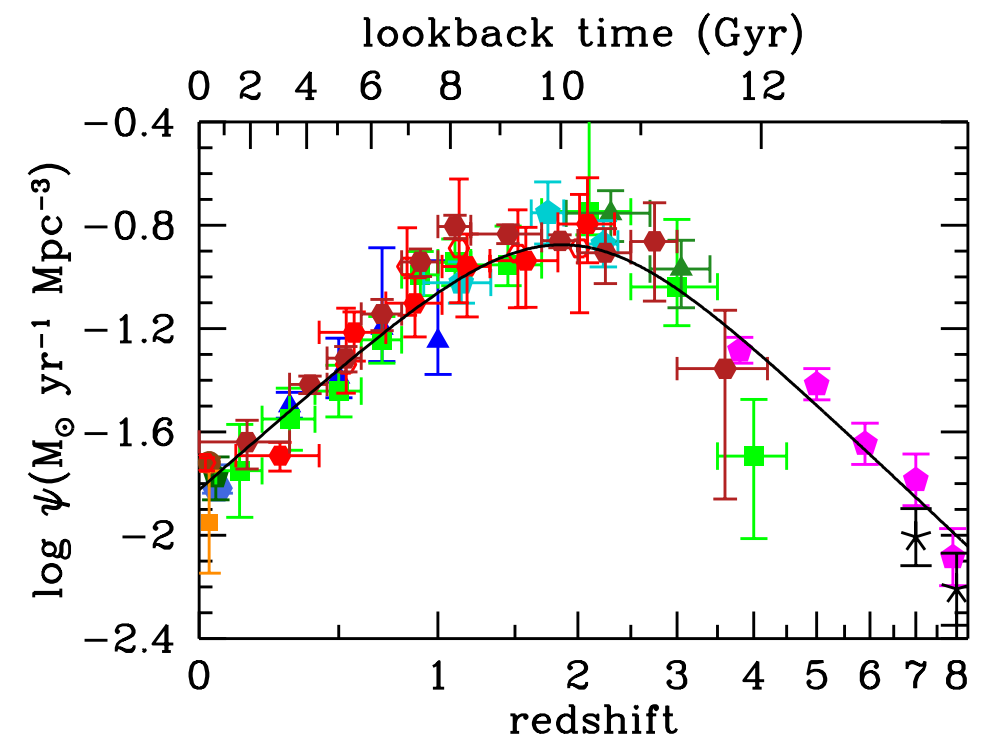

Figure 5.14: The history of cosmic star formation from far UV and infrared restframe measurements. This figure is obtained from Madau \& Dickinson (2014).

of FRBs should follow the star formation history (SFH) of the universe. The cosmic star-formation rate density is peaked at $z \approx 1.9$ and declines exponentially at later times (Madau \& Dickinson, 2014). Figure 5.14 shows the cosmic SFH from UV and IR data and is obtained from Madau \& Dickinson (2014). The best-fitting function for this is given as

$$
\Psi(z)=0.015 \frac{(1+z)^{2.7}}{1+[(1+z) / 2.9]^{5.6}} \mathrm{M}_{\odot} \mathrm{year}^{-1} \mathrm{Mpc}^{-3}
$$

To generate the FRB redshift distribution following cosmic $\mathrm{SFH}$ for some 


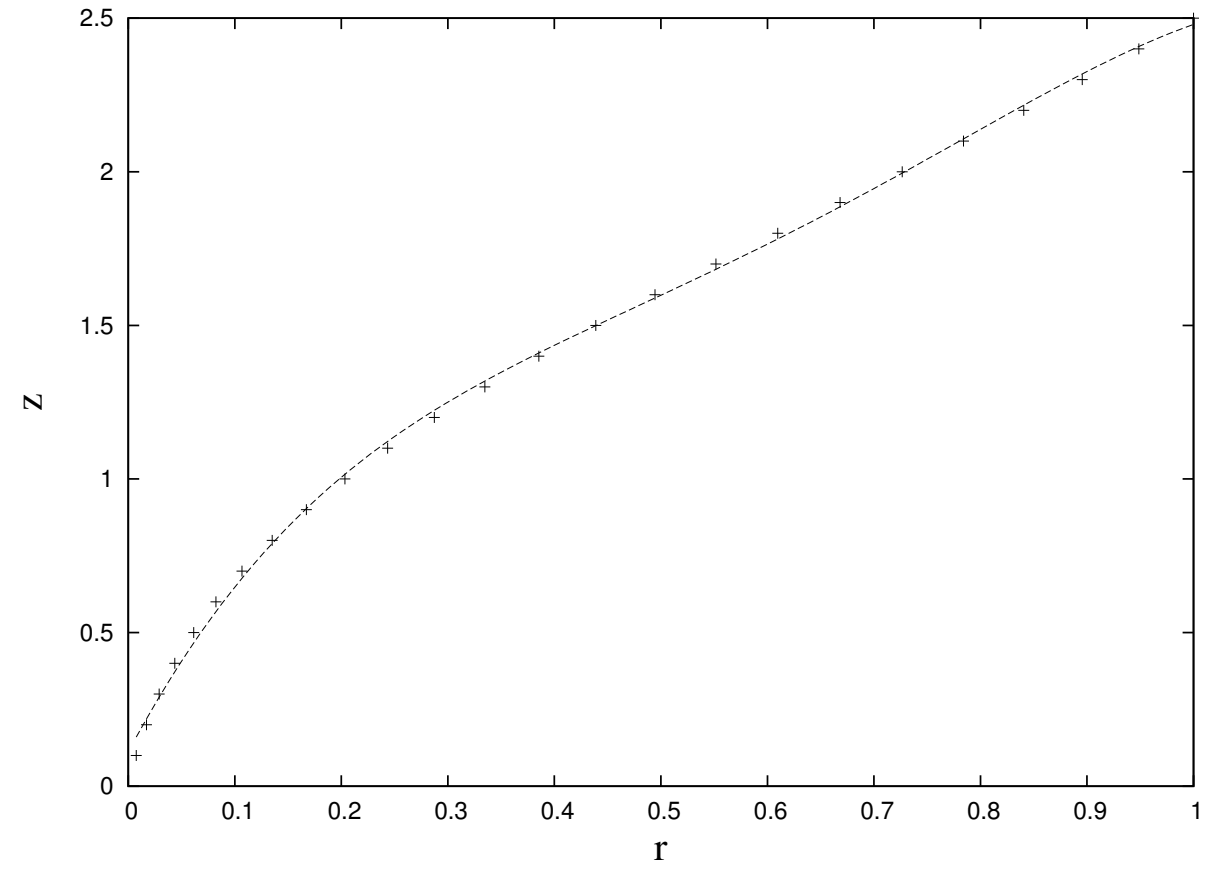

Figure 5.15: The individual points show the redshift distribution of FRBs following cosmic SFH as obtained from Equation 5.30 and the best-fitting curve as dashed lines.

random deviate $r$ we note that

$$
r=\frac{\int_{0}^{z} \Psi\left(z^{\prime}\right) d z^{\prime}}{\int_{0}^{2.5} \Psi\left(z^{\prime}\right) d z^{\prime}}
$$

We plot $z$ as a function of $r$ in Figure 5.15 and find the best-fitting curve to be

$$
z=0.11+6.51 r-12.89 r^{2}+14.48 r^{3}-5.74 r^{4} .
$$

We use this function to generate a redshift distribution of FRBs. In our simulations, we use the optimal values from the best-fit model (model B1) to compute the log likelihood for the SFH model and compare it with the results from the uniform redshift distribution model of FRBs (see Table 5.5). Based on the likelihood values, 
Table 5.5: Optimal parameters and the maximum likelihood for a model in which the redshift distribution tracks the SFH and the constant density model.

\begin{tabular}{lcccc}
\hline Model & \multicolumn{2}{c}{ Parameters } & $\log _{10}(\mathcal{L})$ \\
\hline \multirow{2}{*}{ C2 } & $\sigma_{\mathrm{E}}\left(\times 10^{41} \mathrm{ergs}\right)$ & $L_{\min }\left(\times 10^{42} \mathrm{ergs} \mathrm{s}^{-1}\right)$ & $\alpha_{L}$ & \\
$\mathrm{SFH}$ & 6.0 & 7.8 & 2.2 & -18.9 \\
& 6.0 & 7.8 & 2.2 & -18.4 \\
\hline
\end{tabular}

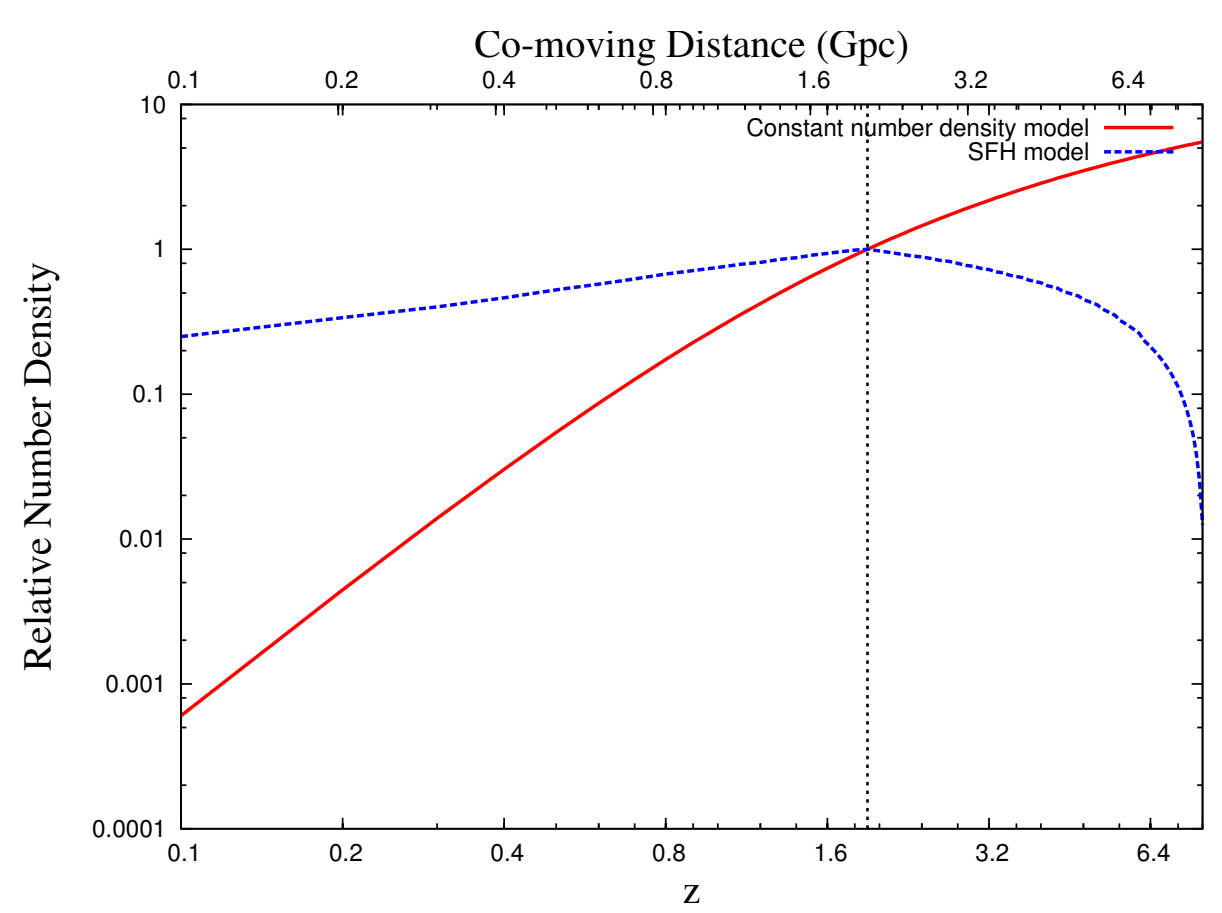

Figure 5.16: The redshift distributions of FRBs tracking cosmic SFH and with constant number density in a co-moving volume.

it can be seen that the two models are indistinguishable for the current sample of FRBs. This is also apparent if we compare the histograms of redshift distributions of simulated FRBs from both the models as shown in Figure 5.16. Although our assumption of uniform distribution of FRBs in a co-moving volume do not affect the results, it should be noted that the two models will differ if we have a larger observed sample of FRBs and if FRBs are discovered at larger redshifts.

To investigate the impacts that a larger sample may have in the future, we performed a Kolmorogov-Smirnov (K-S) test (Press et al., 1992) to compare the two 
Table 5.6: K-S statistic for flux, width, and DM distributions of the two models compared with the known population.

\begin{tabular}{lcc}
\hline Model parameter & \multicolumn{2}{c}{$Q_{\mathrm{KS}}$} \\
& Constant density & Cosmic SFH \\
\hline $\mathrm{DM}$ & 0.02 & 0.83 \\
$W_{\text {obs }}$ & 0.25 & 0.99 \\
$S_{\text {peak }}$ & 0.28 & 0.72 \\
\hline
\end{tabular}

models as a function of number of detectable FRBs. We compared the DM, flux, and width distributions of detectable FRBs from both models and the K-S statistic probability $Q_{\mathrm{KS}}$ as a function of number of samples in each distribution is plotted in Figure 5.17. A criterion of $Q_{\mathrm{KS}}<0.1$ implies that two samples are not drawn from the same distribution. The $Q_{\mathrm{KS}}$ values show that the DM distributions are somewhat distinguishable with even a smaller sample of FRBs. However, we would need an observed sample of at least $100 \mathrm{FRBs}$ to distinguish all three distributions of the two models at the $90 \%$ confidence level as compared to the results from Caleb et al. (2016), which concluded that 50 FRBs are required to distinguish between the two number density models.

We have also performed the K-S test to compare each of the number density models with the known sample of 14 FRBs. The $Q_{\mathrm{KS}}$ values are listed in Table 5.6. Although the results from the flux and width distributions do not distinguish between the two models for the current sample, the much lower K-S probability seen for the DM distribution of model $\mathrm{C} 2$ versus the $\mathrm{SFH}$ model is an indication that FRB progenitors are favoring the redshift distribution that follows the cosmic $\mathrm{SFH}$ rather than being uniformly distributed in a co-moving volume. A larger sample of FRBs will be able to confirm or refute this claim. 


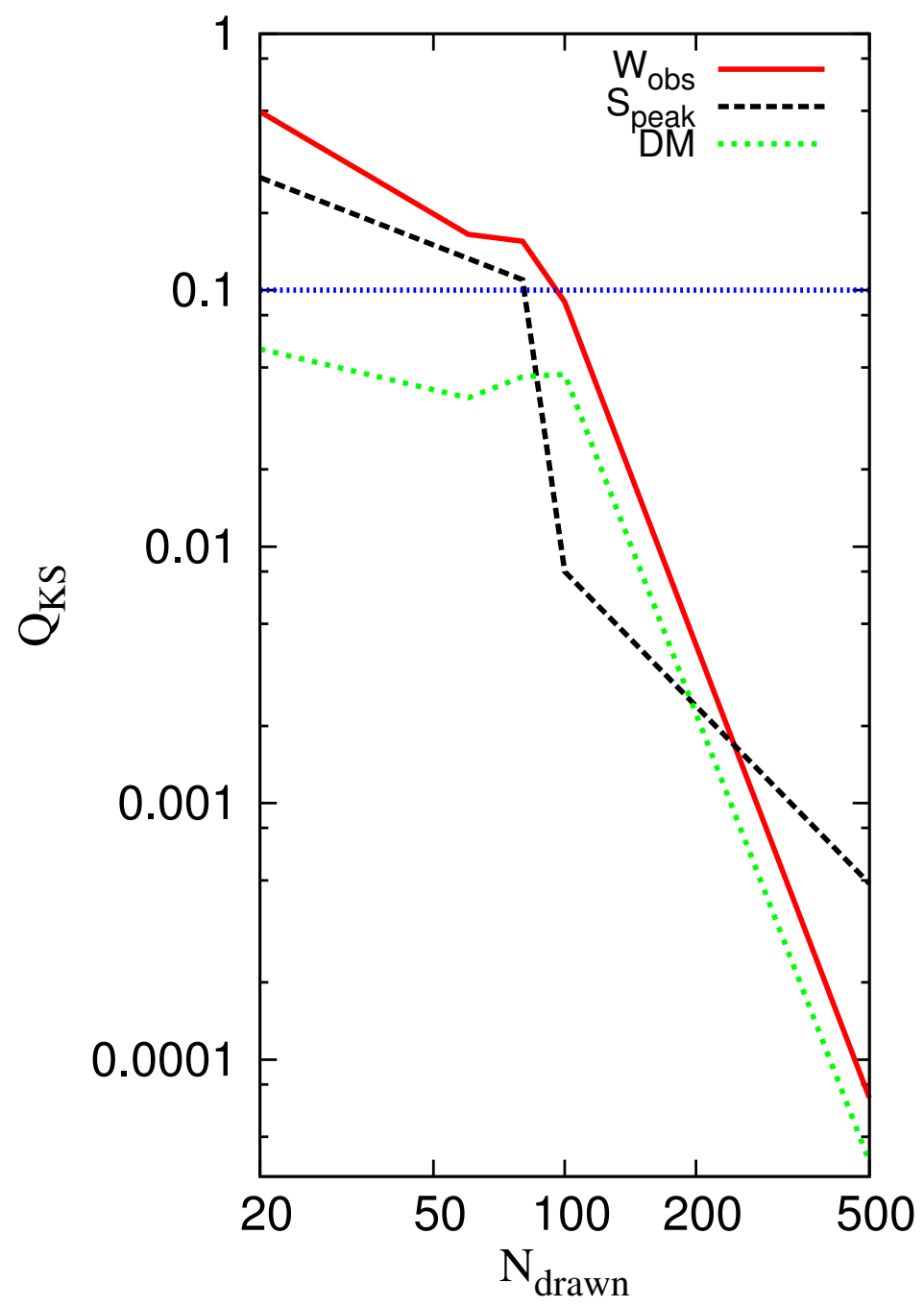

Figure 5.17: Results of the K-S test showing the probability for flux, width, and DM distributions as a function of number of FRBs in each model. 


\subsection{Conclusions}

We have presented simulations of a cosmological population of FRBs that are detectable with the Parkes telescope assuming that FRBs are uniformly distributed in space. We use three distributions for energy and luminosity to generate FRB events in our simulations. The redshift distribution is drawn from their co-moving density. The dispersion measures are derived using the properties of the ISM and IGM. We simulate energy, luminosity, redshift, peak flux, DM, effective width, and signal-to-noise ratio for each FRB and compare this population to the observed FRBs detected at the Parkes telescope using a maximum likelihood analysis.

We find that the best-fit model is the one in which energy has a Gaussian distribution and the luminosity distribution follows a power law. The optimal value of the standard deviation in energy with the $95 \%$ confidence interval is $6.0_{-3.0}^{+4.0} \times 10^{41} \mathrm{ergs}$. The optimal values of the power law luminosity distribution has a minimum luminosity of $7.8_{-0.3}^{+0.3} \times 10^{42} \mathrm{ergs} \mathrm{s}^{-1}$ with a power law index of $2.3_{-0.3}^{+0.3}$. For this set of parameters, the detectable modeled FRB population is consistent with the known population. The models with Gaussian distributed $E$ and $L$ values do not represent the observed FRBs quite well. The need to have a power distribution in luminosity shows that FRBs are unlikely to be standard candles.

For this best-fit model, we compared the results of likelihood for a redshift distribution tracking star formation history and find that the two models are indistinguishable for the current sample of FRBs. We performed a K-S test on the flux,

DM, and width distributions obtained for these 2 models. The results of this test 
show that we would at least need a sample of 100 observed FRBs to distinguish the two models. We also performed a K-S test to compare each of the number density models with the known sample of 14 FRBs. The $Q_{\mathrm{KS}}$ statistic for the DM distributions indicates that FRB progenitors do follow cosmic SFH. We should be able to confirm this suggestion in the future through an analysis of a larger sample of FRBs. 


\section{Chapter 6}

\section{Conclusions and Future Directions}

The work presented in the preceding chapters was based on detecting and understanding fast radio burst phenomena. In Chapter 2, we presented a search for RRATs and FRBs from the Parkes high latitude survey data set. The single-pulse search pipeline was described in detail and was used to search for highly dispersed pulses in the data. This processing resulted in detection of 20 known pulsars and one RRAT but no FRBs were discovered. Since this data were previously processed using the periodicity search, we computed the intermittency measures of these pulsars to compare the two searches based on previous detections and results from our analysis.

Some other archival pulsar surveys carried out with the Parkes telescope were also searched for FRBs previously. We used the null result from our analysis and these results from previous studies to constrain the total event rate of FRBs. In order to do this, we adopted a Bayesian approach which was outlined in Chapter 3. We used results from six surveys and accounting for the different backends used in each survey, total sky coverage and total observation time, we determined that the total event rate of FRBs with a fluence above $4.0 \mathrm{Jy} \mathrm{ms}$ is $\mathcal{R}=4.4_{-3.1}^{+5.2} \times$ $10^{3} \mathrm{FRBs} \mathrm{day}^{-1} \mathrm{sky}^{-1}$ at the $99 \%$ confidence level.

Crawford et al. (2016) processed three additional archival Parkes surveys to search for FRBs and found none. We incorporated these results and using the same 
Bayesian analysis, modified our event rate of FRBs. This resulted in an even lower all-sky rate of $R=3.3_{-2.2}^{+3.7} \times 10^{3}$ events per day per sky above a fluence limit of $3.8 \mathrm{Jy}$ ms at the $99 \%$ confidence level. This result led us to conclude that lack of detections at intermediate latitudes are likely to be due to a lower FRB rate as compared to the rate estimates done previously $\left(\sim 10^{4}\right.$ FRBs per day per sky) and does not necessarily imply a dearth of FRBs at intermediate latitudes as proposed by Petroff et al. (2014). Additionally we predicted the number of expected discoveries in future planned surveys.

Until the end of 2016, no host galaxy was found to be unambiguously associated with an FRB. The thesis also presented a search for host galaxies in Chapter 4 for RRATs that are at the edge of our Galaxy and therefore present a possibility of being an FRB. We found six RRAT candidates that represent this class for which the measured DM value is within the uncertainty of the Galactic free electron density model. We did not find any host galaxy which is within the search beam radius of each of these RRATs. This study suggested that a search for host galaxies should be carried out for future RRAT discoveries near the edge of our Galaxy instead of assuming that they are Galactic sources since these RRATs could possibly be residing in nearby galaxies and therefore could be FRBs. In Chapter 5, we investigated the FRB population by carrying out Monte Carlo simulations of FRBs which have different energy and luminosity distributions. From a maximum likelihood analysis, we found that the known population of FRBs can be best expressed as having Gaussian distributed energy values and has a luminosity distribution that is a power law. We found the optimal value of the standard deviation in energy with the $95 \%$ confidence 
interval to be $6.0_{-3.0}^{+4.0} \times 10^{41} \mathrm{ergs}$. The optimal values of the power law luminosity distribution was found to have a minimum luminosity of $7.8_{-0.3}^{+0.3} \times 10^{42} \mathrm{ergs} \mathrm{s}^{-1}$ with a power law index of $2.3_{-0.3}^{+0.3}$. The mean energy and luminosity of this distribution were determined to be $4.7 \times 10^{41} \mathrm{ergs}$ and $1.1 \times 10^{45} \mathrm{ergs} \mathrm{s}^{-1}$ respectively. We concluded that the need to have a power law luminosity distribution implies that FRBs are unlikely to be standard candles. Our main assumption in this analysis was that FRBs are uniformly distributed in the sky. For the best-fit model, we simulated a distribution that followed star formation history in which the number density peaks at a redshift of $\sim 1.9$. We determined that for the current sample of FRBs, we cannot differentiate between the constant density model in which FRBs are uniformly distributed and the model in which FRBs follow star formation history. The results of the K-S test showed that we would require at least 100 FRBs to distinguish these two models. A second K-S test performed to compare each of the number density models with the known sample of 14 FRBs shows that FRB progenitors do follow cosmic SFH at least from the DM distributions. More discoveries in the near future will be able to confirm or refute this claim.

All of the results presented in this thesis provided a new study of this population while exploring the parameter space of their properties. However, there are still many questions, particularly about the origin of the FRB pulses, that remain unsolved. As discussed in the introduction, the true distances to their progenitors and their usefulness as probes of the intervening ionized material remain to be determined. The current population is not sufficient to perform population statistics studies, and therefore, we need a larger population of FRBs. Given the relatively 
high all-sky rate inferred in Chapter 3, a telescope with a large field of view, a large amount of time on sky, and high sensitivity would be able to find a large number of FRBs. In addition to the existing telescopes, a number of new powerful radio telescopes will begin searching for these highly dispersed bursts in the near future which are predicting much higher detection rates. For example, the Canadian Hydrogen Intensity Mapping Experiment telescope (CHIME; Newburgh et al., 2014) is expecting to detect a few FRBs per day (Rajwade \& Lorimer, 2017; CHIME Scientific Collaboration et al., 2017). Also, precursor telescopes for the Square Kilometre Array (SKA) such the Australia SKA Pathfinder (ASKAP), MeerKAT, the Molonglo radio telescope (UTMOST) will enable us to find a larger sample of FRBs. Going forward, follow-up for FRBs and detailed statistical studies of the FRB population will be important to place further constraints on progenitor models and eventually to have a better understanding of FRBs. 


\section{Appendix A}

\section{Pulse broadening due to DM step size}

Levin (2012) describes the the algorithm which accounts for the amount of pulse broadening caused by the size of the previous DM step and then determines the next trial DM. The appropriate step size is computed from the effective pulse width $W_{\text {eff }}$, which is a quadrature sum of the intrinsic pulse width $t_{\text {in }}$, the smearing due to scattering $t_{\text {scatt }}$, dispersion smearing within each frequency channel $t_{\mathrm{DM}}$, sampling time of a particular survey $t_{\text {samp }}$, and smearing across all frequency channels due to the dispersion measure step size $t_{\Delta \mathrm{DM}}$ :

$$
W_{\text {eff }}=\sqrt{t_{\text {in }}^{2}+t_{\mathrm{scatt}}^{2}+t_{\mathrm{samp}}^{2}+t_{\mathrm{DM}}^{2}+t_{\Delta \mathrm{DM}}^{2}}
$$

where, for frequencies in $\mathrm{GHz}$ and $\mathrm{DM}$ in units of $\mathrm{cm}^{-3} \mathrm{pc}$,

$$
t_{\mathrm{DM}}=8300 \mathrm{~s}\left(\frac{\Delta \nu \mathrm{DM}}{\nu^{3}}\right)
$$

and

$$
t_{\Delta \mathrm{DM}}=8300 \mathrm{~s}\left(\frac{n_{\text {chan }} \Delta \nu \Delta \mathrm{DM}}{4 \nu^{3}}\right) .
$$

Here $n_{\text {chan }}$ is the number of frequency channels and $\Delta \mathrm{DM}=\mathrm{DM}-\mathrm{DM}^{\prime}$. Here DM is the new trial $\mathrm{DM}$ value and $\mathrm{DM}^{\prime}$ is the last trial $\mathrm{DM}$ value. Since 4 samples are packed per 64-bit word, the number of channels is divided by a factor of 4 . Since 
the total pulse width smearing of $25 \%$ is chosen, the pulse broadening fraction due to the DM step is $\epsilon=1.25$. Then the effective pulse width at the new trial DM with respect to the last trial $\mathrm{DM}^{\prime}$ is

$$
\left(W_{\mathrm{eff} \mid \mathrm{DM}}\right)=\epsilon \quad\left(W_{\mathrm{eff} \mid \mathrm{DM}^{\prime}}\right)
$$

Solving for DM by equating $W_{\text {eff }}$ at the new trial value, DM, with respect to the last trial value, $\mathrm{DM}^{\prime}$ gives,

$$
\mathrm{DM}=\frac{b^{2} \mathrm{DM}^{\prime}+\sqrt{-a^{2} b^{2} \mathrm{DM}^{\prime 2}+a^{2} c+b^{2} c}}{a^{2}+b^{2}}
$$

where

$$
\begin{gathered}
a=8300 \mathrm{~s} \frac{\Delta \nu \mathrm{DM}}{\nu^{3}}, \\
b=t_{\Delta \mathrm{DM}}=8300 \mathrm{~s} \frac{n_{\text {chan }} \Delta \nu \Delta \mathrm{DM}}{4 \nu^{3}},
\end{gathered}
$$

and

$$
c=\epsilon^{2}\left(t_{\mathrm{in}}^{2}+t_{\mathrm{scatt}}^{2}+t_{\mathrm{samp}}^{2}+t_{\mathrm{DM}}^{2}\right)-t_{\mathrm{samp}}^{2}-W_{\mathrm{in}}^{2} .
$$

The DM values are obtained using Equation A.5 where the DM step is finely spaced for small values of DM. For higher DM values, pulse smearing is greater than the sampling time and hence the temporal resolution is decreased giving DM channels that are spaced more coarsely. 


\section{Appendix B}

\section{Contribution to DM from the intergalactic medium}

As mentioned in $\S 5.5$, FRBs are cosmological sources and originate in some host galaxy at a redshift of $z$. As the radiation travels from the host galaxy to the telescope, it gets dispersed. This dispersion is a function of redshift and different dispersive regions will contribute to the total DM observed at the telescope

$$
\mathrm{DM}_{\mathrm{observed}}=\mathrm{DM}_{\mathrm{MW}}+\mathrm{DM}_{\mathrm{IGM}}+\mathrm{DM}_{\text {host }}
$$

where $\mathrm{DM}_{\mathrm{MW}}$ is the dispersion measure within the Milky Way arising due to the ISM, $\mathrm{DM}_{\mathrm{IGM}}$ is due to the IGM, and $\mathrm{DM}_{\text {host }}$ is the DM due to the turbulent medium in the host galaxy of the source. We derive the IGM contribution here.

The electron density in the IGM is

$$
n_{\mathrm{e}, \mathrm{IGM}}=x(z) n_{\mathrm{e}, 0}(1+z)^{3},
$$

where $x(z)$ is the ionization fraction and $n_{\mathrm{e}, 0}=2 \times 10^{-7} \mathrm{~cm}^{-3}$ is the mean local inter-galactic electron density. This gives

$$
\mathrm{DM}_{\mathrm{IGM}}=\int_{0}^{L} n_{\mathrm{e}, \mathrm{IGM}} d l .
$$


For this cosmological application, the conversion from $d l$ to $d z$ is as follows:

$$
d l=c \frac{d t}{d z} d z
$$

And

$$
\frac{d t}{d z}=\frac{1}{1+z} \frac{1}{H(z)}
$$

where $H(z)$ is the Hubble constant. In a $\Lambda$-CDM model,

$$
H(z)=H_{0}\left(\sqrt{\Omega_{m}(1+z)^{3}+\Omega_{\Lambda}}\right),
$$

where $H_{0}=68 \mathrm{~km} \mathrm{~s}^{-1} \mathrm{Mpc}^{-1}$ is the Hubble constant at present, and $\Omega_{m}=0.32$, $\Omega_{\Lambda}=0.68$ are the fractions of baryonic matter density and dark matter density for a flat universe respectively.

Following Zheng et al. (2014), the propagation time $t$ of a photon traveling through the IGM to reach the observer is affected by the continuous change of photon frequency with redshift, the change of the plasma frequency $\nu_{\mathrm{p}}$ due to the change in the IGM electron density with redshift, and the time dilation effect. So, the propagation time of a photon emitted at redshift $z$ seen by an observer at redshift 0 is

$$
t=\int_{0}^{z} \frac{d l}{d z} \frac{1}{v_{\mathrm{g}}}(1+z)
$$

where the group velocity is

$$
v_{\mathrm{g}}=c \sqrt{1-\frac{\nu_{\mathrm{p}}^{2}}{\nu^{\prime 2}}}
$$


The plasma frequency is

$$
\nu_{\mathrm{p}}=\sqrt{\frac{n_{\mathrm{e}} e^{2}}{\pi m_{\mathrm{e}}}} .
$$

and $\nu^{\prime}=\nu(1+z)$ is the rest frame frequency. This implies

$$
t=\frac{1}{c} \int_{0}^{z} d z \frac{c}{(1+z) H(z)}\left(1+\frac{1}{2} \frac{\nu_{p}^{2}}{\nu^{\prime 2}}\right)(1+z) .
$$

From Equation B.3, we have

$$
t=\frac{e^{2}}{2 \pi m_{\mathrm{e}} c} \frac{\mathrm{DM}_{\mathrm{IGM}}}{\nu^{2}} .
$$

Therefore,

$$
\frac{d t}{d \nu}=-\frac{4 \pi e^{2}}{\nu^{3} m_{\mathrm{e}} c} \mathrm{DM}_{\mathrm{IGM}}
$$

Substituting for $t$ from Equation B.10,

$$
\begin{aligned}
& \frac{d t}{d \nu}=\int_{0}^{z} d z \frac{1}{H(z)}\left[\frac{d}{d \nu}\left(1+\frac{1}{2} \frac{\nu_{p}^{2}}{\nu^{\prime 2}}\right)\right] \\
& \Longrightarrow \frac{d t}{d \nu}=-\int_{0}^{z} d z \frac{1}{(1+z)^{2} H(z)} \frac{\nu_{\mathrm{p}}^{2}}{\nu^{3}} .
\end{aligned}
$$

Thus we get

$$
\begin{gathered}
\mathrm{DM}_{\mathrm{IGM}}=\frac{m_{\mathrm{e}} c}{4^{2}} \int_{0}^{z} d z \frac{\nu_{\mathrm{p}}^{2}}{(1+z)^{2} H(z)} \\
\mathrm{DM}_{\mathrm{IGM}}=\frac{\mathrm{m}_{\mathrm{e}} \mathrm{c}}{4^{2}} \frac{4 \pi \mathrm{n}_{\mathrm{e}, 0}}{\mathrm{e}} m_{\mathrm{e}} \int_{0}^{z} d z \frac{x(z)(1+z)^{3}}{(1+z)^{2} H(z)}
\end{gathered}
$$


$\Longrightarrow \mathrm{DM}_{\mathrm{IGM}}=\frac{c}{H_{0}} n_{\mathrm{e}, 0} \int_{0}^{z} d z \frac{(1+z) x(z)}{\sqrt{\Omega_{\mathrm{m}}(1+z)^{3}+\Omega_{\Lambda}}}$. 


\section{Appendix C}

\section{MCMC results for all models}

Following the Markov chain explained in $\S 5.10$, we perform 6000 iterations for each of three models considered in our analysis. Model A refers to FRBs having a Gaussian distributed energy and luminosity distribution. In model B, FRBs have a constant energy and a power law distribution of luminosities. Model C refers to FRBs with a power law distribution of luminosities and Gaussian distributed energies. "1" after each model corresponds to models in which there is no scattering within the Milky Way and the host galaxy alone contributes to the scattering. "2" after each model corresponds to models in which scattering contribution is due to Milky Way and the host galaxy.

Here we show the MCMC results for the free parameters in all 6 models. The left panel are traceplots showing the running mean of each parameter over the entire 6000 iterations of the MCMC. The region before the dashed vertical line represents the burn-in period. The marginalized PDFs for each parameter are constructed after discarding the steps before this burn-in period and are shown in the right panels. 

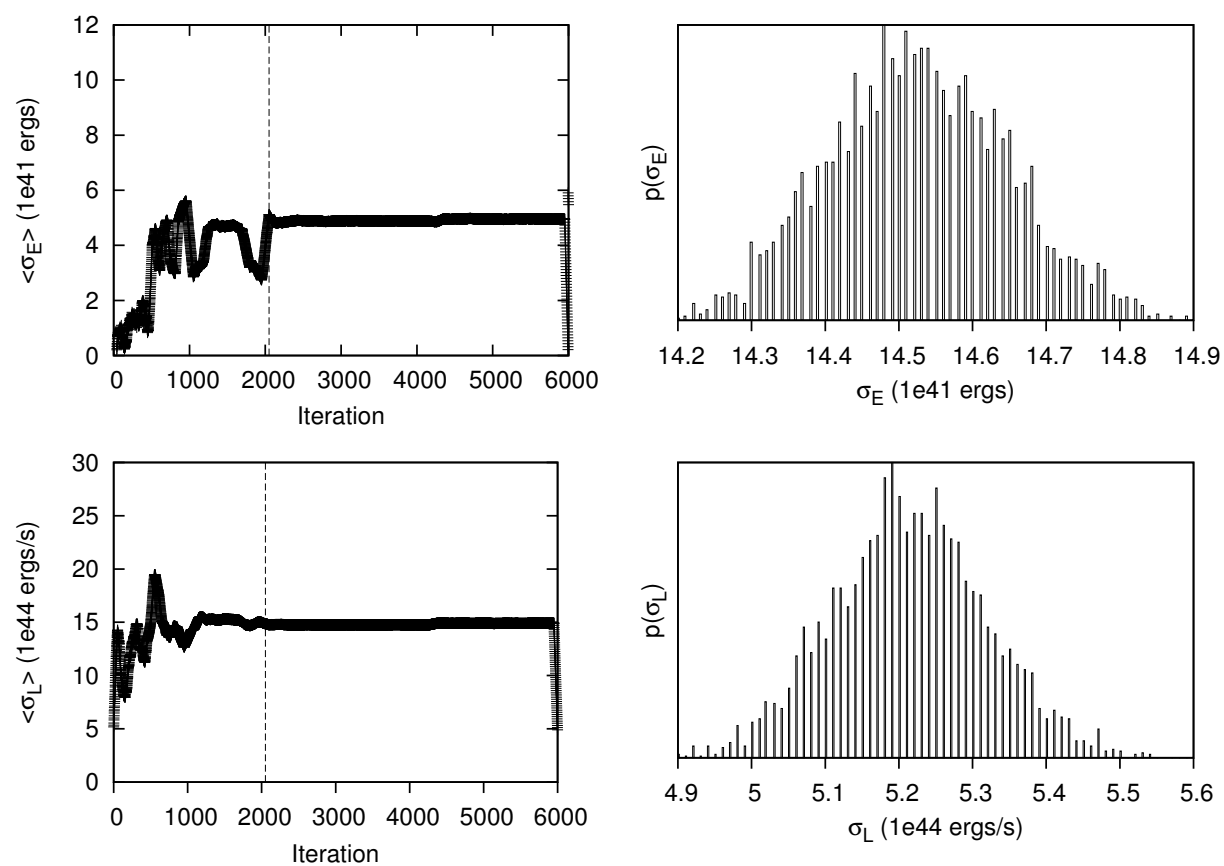

Figure C.1: MCMC results for model A1 with scattering due to the host galaxy alone.
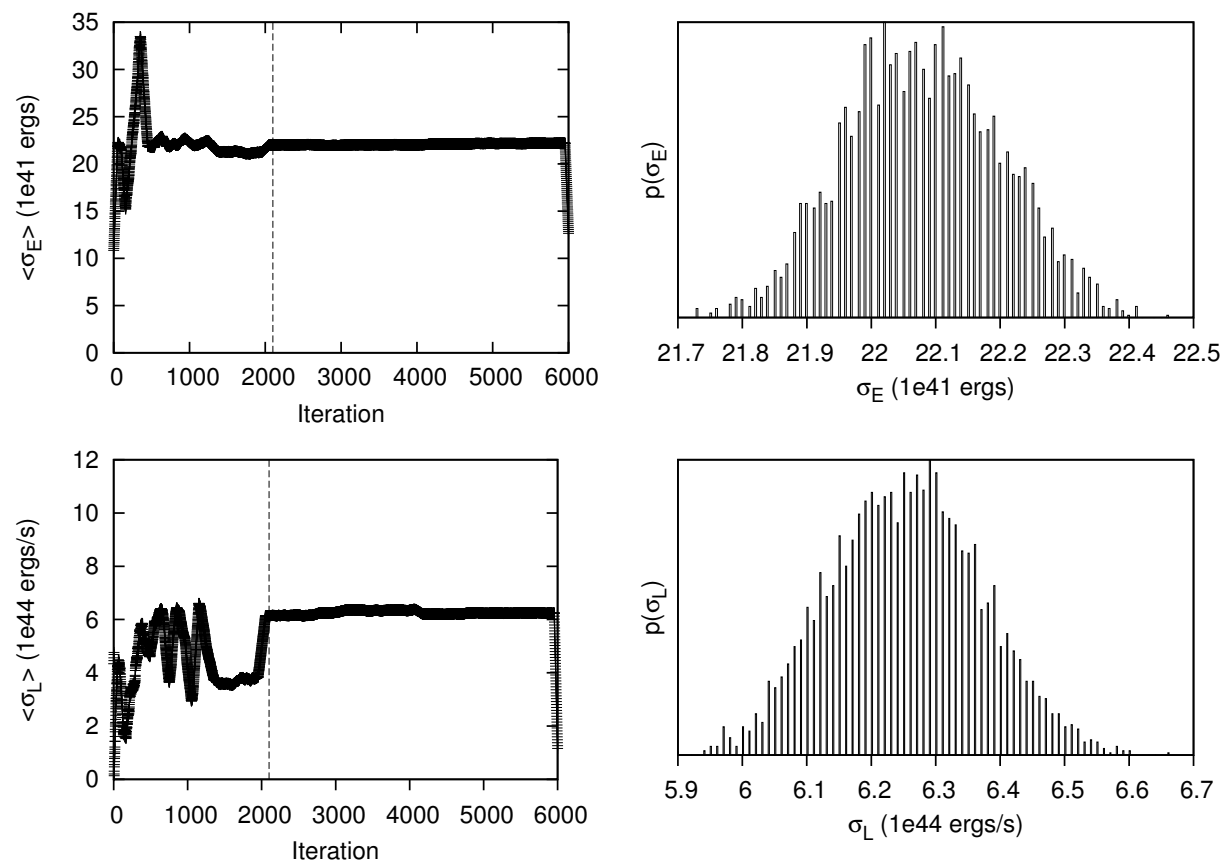

Figure C.2: MCMC results for model A2 with scattering due to the Milky Way and the host galaxy. 

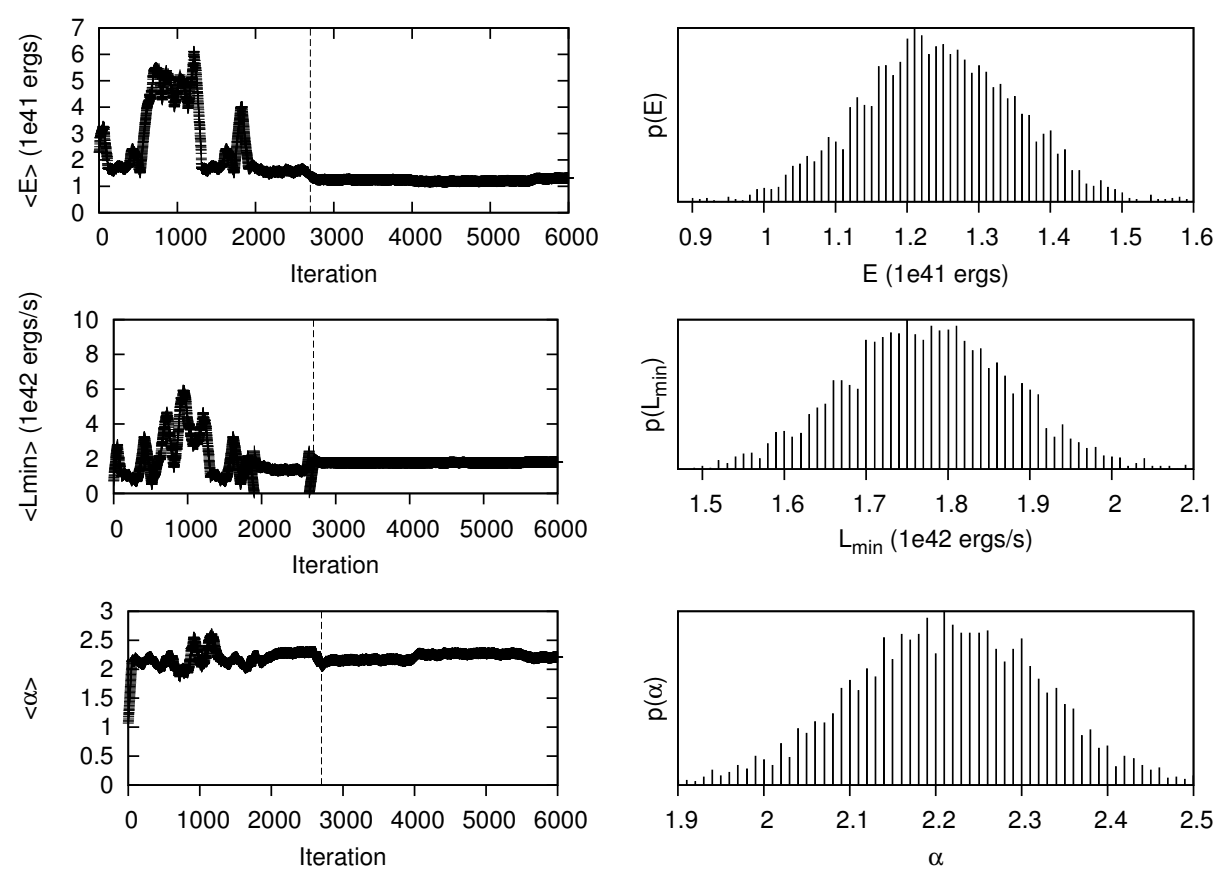

Figure C.3: MCMC results for model B1 with scattering due to the host galaxy alone.
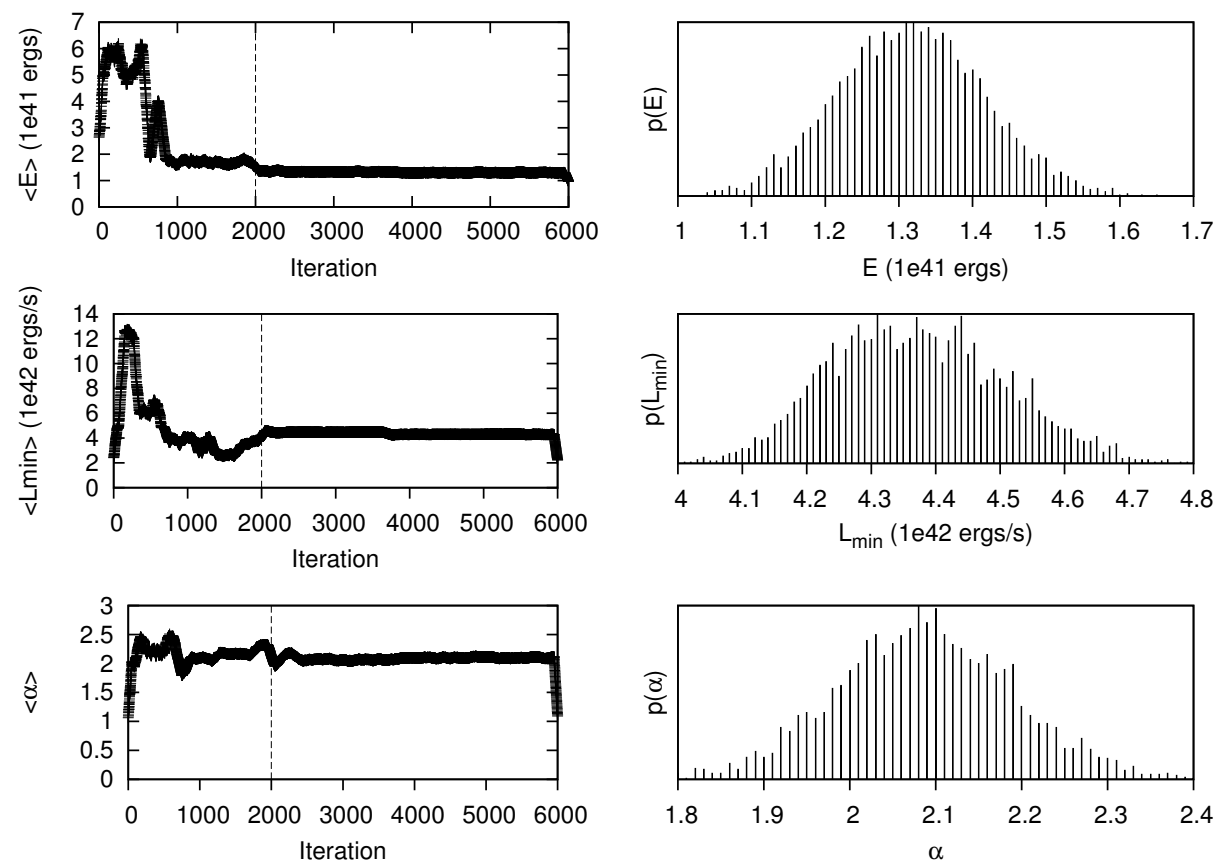

Figure C.4: MCMC results for model B2 with scattering due to the Milky Way and the host galaxy. 

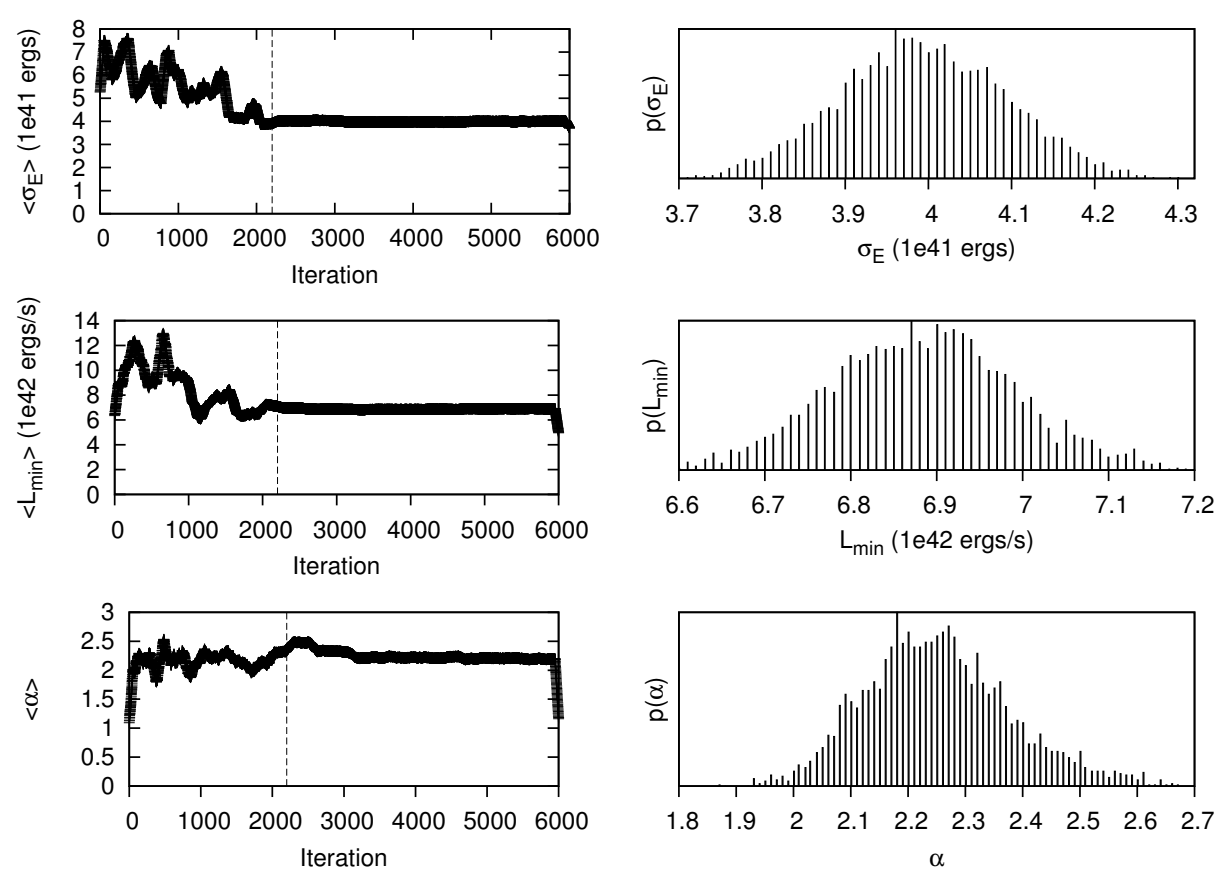

Figure C.5: MCMC results for model $\mathrm{C} 1$ with scattering due to the host galaxy alone.
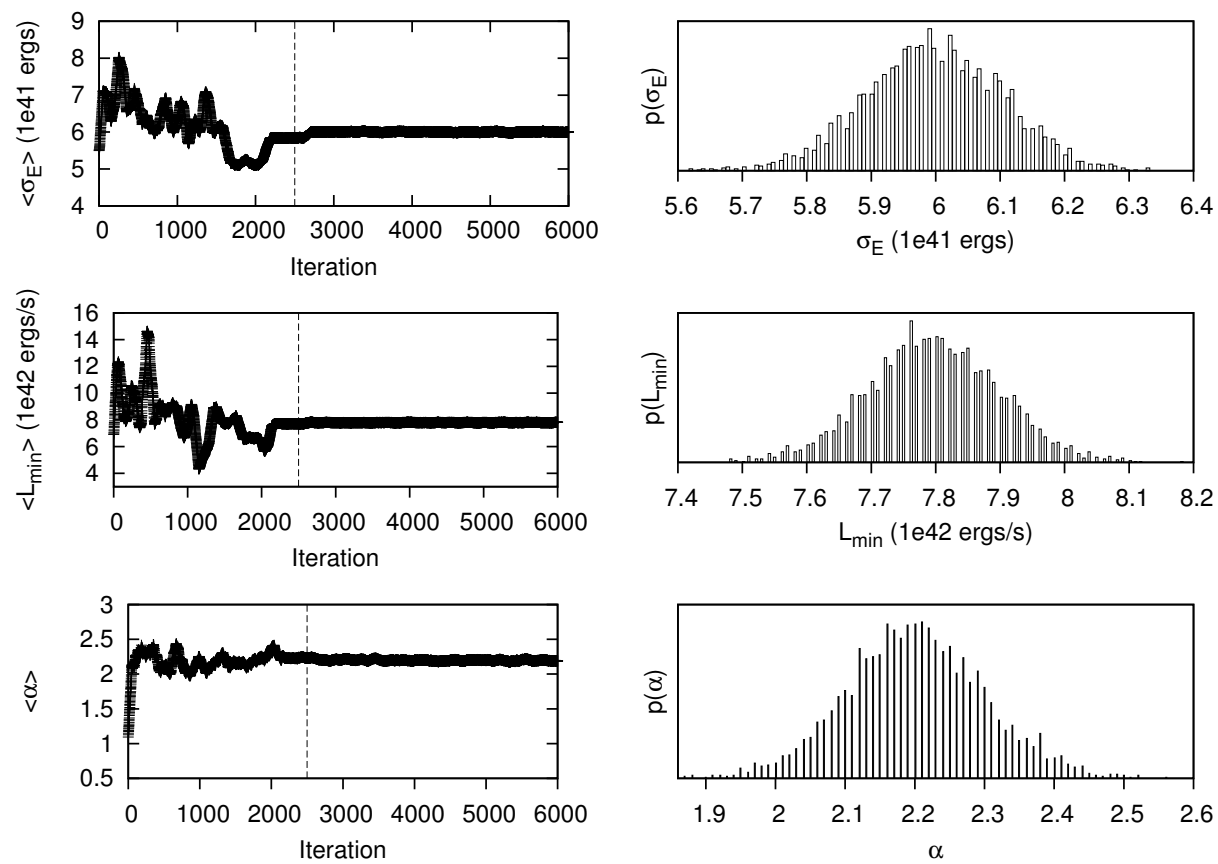

Figure C.6: MCMC results for model C2 with scattering due to the Milky Way and the host galaxy. 


\section{Bibliography}

Abdo, A. A., Ackermann, M., Ajello, M., et al. 2009, Physical Review Letters, 103, 251101

Planck Collaboration, Ade, P. A. R., Aghanim, N., et al. 2016, A\&A, 594, A13

CHIME Scientific Collaboration, Amiri, M., Bandura, K., et al. 2017, arXiv:1702.08040

Akahori, T., Ryu, D., \& Gaensler, B. M. 2016, ApJ, 824, 105

Appleton, E. V. 1945, Nature, 156, 534

Backer, D. C., Kulkarni, S. R., Heiles, C., Davis, M. M., \& Goss, W. M. 1982, Nature, 300, 615

Bagchi, M., Nieves, A. C., \& McLaughlin, M. 2012, MNRAS, 425, 2501

Bannister, K. W., \& Madsen, G. J. 2014, MNRAS, 440, 353

Bannister, K. W., Shannon, R. M., Macquart, J.-P., et al. 2017, ApJL, 841, L1

Bera, A., Bhattacharyya, S., Bharadwaj, S., Bhat, N. D. R., \& Chengalur, J. N. 2016, MNRAS, 457, 2530

Bhat, N. D. R., Cordes, J. M., Camilo, F., Nice, D. J., \& Lorimer, D. R. 2004, ApJ, 605,759

Bonetti, L., Ellis, J., Mavromatos, N. E., et al. 2016, Physics Letters B, 757, 548 
Bonetti, L., Ellis, J., Mavromatos, N. E., et al. 2017, arXiv:1701.03097

Burgay, M., Joshi, B. C., D'Amico, N., et al. 2006, MNRAS, 368, 283

Burgay, M., Keith, M. J., Lorimer, D. R., et al. 2013, MNRAS, 429, 579

Burke-Spolaor, S., Bailes, M., Ekers, R., Macquart, J.-P., \& Crawford, F., III 2011, ApJ, 727, 18

Burke-Spolaor, S., Bailes, M., Johnston, S., et al. 2011, MNRAS, 416, 2465

Burke-Spolaor S., Bannister K. W., 2014, ApJ, 792, 19

Caleb, M., Flynn, C., Bailes, M., et al. 2016, MNRAS, 458, 708

https://arxiv.org/abs/1703.10173

Champion, D. J., Petroff, E., Kramer, M., et al. 2016, MNRAS, 460, L30

Chatterjee, S., Law, C. J., Wharton, R. S., et al. 2017, arXiv:1701.01098

Cognard, I., Shrauner, J. A., Taylor, J. H., \& Thorsett, S. E. 1996, ApJL, 457, L81

Colless, M., Dalton, G., Maddox, S., et al. 2001, MNRAS, 328, 1039

Connor, L., Sievers, J., \& Pen, U.-L. 2016, MNRAS, 458, L19

Cordes, J. M., \& Rickett, B. J. 1998, ApJ, 507, 846

Cordes, J. M., \& Lazio, T. J. W. 2002, arXiv:astro-ph/0207156

Cordes, J. M., \& McLaughlin, M. A. 2003, ApJ, 596, 1142 
Cordes, J. M., Lazio, T. J. W., \& McLaughlin, M. A. 2004, New Astronomy Review, 48,1459

Cordes, J. M., Freire, P. C. C., Lorimer, D. R., et al. 2006, ApJ, 637, 446

Cordes, J. M., \& Wasserman, I. 2016, MNRAS, 457, 232

Cordes, J. M., Wharton, R. S., Spitler, L. G., Chatterjee, S., \& Wasserman, I. 2016, arXiv:1605.05890

Crawford, F., Rane, A., Tran, L., et al. 2016, MNRAS, 460, 3370

DeLaunay, J. J., Fox, D. B., Murase, K., et al. 2016, ApJL, 832, L1

Deneva, J. S., Cordes, J. M., McLaughlin, M. A., et al. 2009, ApJ, 703, 2259

Deneva, J. S., Stovall, K., McLaughlin, M. A., et al. 2016, ApJ, 821, 10

Dennison, B. 2014, MNRAS, 443, L11

Dolag, K., Gaensler, B. M., Beck, A. M., \& Beck, M. C. 2015, MNRAS, 451, 4277

Duncan, R. C., \& Thompson, C. 1992, ApJL, 392, L9

Dulk, G. A. 1985, ARA\&A, 23, 169

Eatough, R. P., Keane, E. F., \& Lyne, A. G. 2009, MNRAS, 395, 410

Edwards, R. T., Bailes, M., van Straten, W., \& Britton, M. C. 2001, MNRAS, 326, 358

Falcke, H., \& Rezzolla, L. 2014, A\&A, 562, AA137 
Falco, E. E., Kurtz, M. J., Geller, M. J., et al. 1999, PASP, 111, 438

Farrell, W. M., Desch, M. D., \& Zarka, P. 1999, , 104, 14025

Gaensler, B. M., Madsen, G. J., Chatterjee, S., \& Mao, S. A. 2008, PASA, 25, 184

Gold, T. 1968, Nature, 218, 731

Gomez-Gonzalez, J., \& Guelin, M. 1974, A\&A, 32, 441

Gregory, P. C. 2005, Bayesian Logical Data Analysis for the Physical Sciences: A Comparative Approach with 'Mathematica' Support. Edited by P. C. Gregory. ISBN 052184150 X (hardback); QA279.5.G74 2005 519.5’42 - dc22; 200445930. Published by Cambridge University Press, Cambridge, UK, 2005.,

Hallinan, G., Bourke, S., Lane, C., et al. 2007, ApJL, 663, L25

Hallinan, G., Antonova, A., Doyle, J. G., et al. 2008, ApJ, 684, 644-653

Hastings, W. (1970). Monte Carlo sampling methods using Markov chains and their application. Biometrika, 57: 97109.

Hessels, J. W. T., Ransom, S. M., Stairs, I. H., et al. 2006, Science, 311, 1901

Hewish, A., Bell, S. J., Pilkington, J. D. H., Scott, P. F., \& Collins, R. A. 1968, Nature, 217, 709

Hogg, D. W. 1999, arXiv:astro-ph/9905116

Huege, T., \& Falcke, H. 2003, A\&A, 412, 19 
Hulse, R. A., \& Taylor, J. H. 1974, ApJL, 191, L59

Ioka, K. 2003, ApJL, 598, L79

Inoue, A. K., Yamazaki, R., \& Nakamura, T. 2004, ApJ, 601, 644

Jacoby, B. A., Bailes, M., Ord, S. M., Edwards, R. T., \& Kulkarni, S. R. 2009, ApJ, 699, 2009

Karako-Argaman, C., Kaspi, V. M., Lynch, R. S., et al. 2015, ApJ, 809, 67

Kashiyama, K., Ioka, K., \& Mészáros, P. 2013, ApJl, 776, LL39

Kaspi, V. M., \& Beloborodov, A. 2017, arXiv:1703.00068

Keane, E. F., Ludovici, D. A., Eatough, R. P., et al. 2010, MNRAS, 401, 1057

Keane, E. F., Kramer, M., Lyne, A. G., Stappers, B. W., \& McLaughlin, M. A. 2011, MNRAS, 415, 3065

Keane, E. F., Stappers, B. W., Kramer, M., \& Lyne, A. G. 2012, MNRAS, 425, L71

Keane, E. F., \& Petroff, E. 2015, MNRAS, 447, 2852

Keane, E. F., Johnston, S., Bhandari, S., et al. 2016, Nature, 530, 453

Kocz, J., Bailes, M., Barnes, D., Burke-Spolaor, S., \& Levin, L. 2012, MNRAS, 420, 271

Kouwenhoven, M. L. A., \& Voûte, J. L. L. 2001, A\&A, 378, 700

Kramer, M. 2016, International Journal of Modern Physics D, 25, 1630029-61 
Lazarus, P., Brazier, A., Hessels, J. W. T., et al. 2015, ApJ, 812, 81

Lazio, T. J., W., Farrell, W. M., Dietrick, J., et al. 2004, ApJ, 612, 511

Lazio, T. J. W., Ojha, R., Fey, A. L., et al. 2008, ApJ, 672, 115-121

Lazio, T. J. W. 2012, Square Kilometre Array: Paving the Way for the New 21st Century Radio Astronomy Paradigm, 53

Levin L., 2012, PhD thesis, Swinburne University of Technology

Liebling, S. L., \& Palenzuela, C. 2016, Phys. Rev. D, 94, 064046

Lipunov, V. M., \& Pruzhinskaya, M. V. 2014, MNRAS, 440, 1193

Liu, T., Romero, G. E., Liu, M.-L., \& Li, A. 2016, ApJ, 826, 82

Loeb, A., Shvartzvald, Y., \& Maoz, D. 2014, MNRAS, 439, L46

Lorimer, D. R., Bailes, M., Dewey, R. J., \& Harrison, P. A. 1993, MNRAS, 263, 403

Lorimer, D. R., \& Kramer, M. 2004, Handbook of pulsar astronomy, by D.R. Lorimer and M. Kramer. Cambridge observing handbooks for research astronomers, Vol. 4. Cambridge, UK: Cambridge University Press, 2004

Lorimer, D. R., Faulkner, A. J., Lyne, A. G., et al. 2006, MNRAS, 372, 777

Lorimer, D. R., Bailes, M., McLaughlin, M. A., Narkevic, D. J., \& Crawford, F. 2007, Science, 318, 777

Lorimer, D. R. 2008, Living Reviews in Relativity, 11, 
Lorimer, D. R., \& McLaughlin, M. A. 2010, Highlights of Astronomy, 15, 131

Lorimer, D. R., Karastergiou, A., McLaughlin, M. A., \& Johnston, S. 2013, MNRAS, 436, L5

Luan, J., \& Goldreich, P. 2014, ApJl, 785, LL26

Lyne, A. G., \& Rickett, B. J. 1968, Nature, 219, 1339

Lyutikov, M. 2002, ApJL, 580, L65

Lyutikov, M. 2006, MNRAS, 367, 1594

Lyutikov, M., \& Lorimer, D. R. 2016, ApJL, 824, L18

Macquart, J.-P., \& Koay, J. Y. 2013, ApJ, 776, 125

Macquart, J.-P., \& Johnston, S. 2015, MNRAS, 451, 3278

Madau, P., \& Dickinson, M. 2014, ARA\&A, 52, 415

Manchester, R. N., Lyne, A. G., Camilo, F., et al. 2001, MNRAS, 328, 17

Manchester, R. N., Hobbs, G. B., Teoh, A., \& Hobbs, M. 2005, AJ, 129, 1993-2006 (2005), astro-ph/0412641

Maoz, D., Loeb, A., Shvartzvald, Y., et al. 2015, arXiv:1507.01002

Marcote, B., Paragi, Z., Hessels, J. W. T., et al. 2017, ApJL, 834, L8

Masui, K., Lin, H.-H., Sievers, J., et al. 2015, Nature, 528, 523 
Mazets, E. P., Golenetskij, S. V., \& Guryan, Y. A. 1979, Soviet Astronomy Letters, 5,641

Mazets, E. P., Golentskii, S. V., Ilinskii, V. N., Aptekar, R. L., \& Guryan, I. A. 1979, Nature, 282, 587

Mazets, E. P., \& Golenetskii, S. V. 1981, Ap\&SS, 75, 47

McIntosh, D. H., Bell, E. F., Rix, H.-W., et al. 2005, ApJ, 632, 191

McLaughlin, M. A., \& Cordes, J. M. 2003, ApJ, 596, 982

McLaughlin, M. A., Lyne, A. G., Lorimer, D. R., et al. 2006, Nature, 439, 817

McQuinn, M. 2014, ApJL, 780, L33

McQuinn, M., Lidz, A., Zaldarriaga, M., et al. 2009, ApJ, 694, 842

Metropolis, N., Rosenbluth, A., Rosenbluth, M., Teller, A., and Teller, E. (1953). Equations of state calculations by fast computing machines. J. Chem. Phys., 21(6): 10871092.

Metzger, B. D., Berger, E., \& Margalit, B. 2017, arXiv:1701.02370

Miller, M. J., \& Bregman, J. N. 2015, ApJ, 800, 14

Mingarelli, C. M. F., Levin, J., \& Lazio, T. J. W. 2015, ApJL, 814, L20

Murase, K., Kashiyama, K., \& Mészáros, P. 2016, MNRAS, 461, 1498

Nicastro, F., Mathur, S., \& Elvis, M. 2008, Science, 319, 55 
Oppermann, N., Connor, L. D., \& Pen, U.-L. 2016, MNRAS, 461, 984

Özel, F., \& Freire, P. 2016, ARA\&A, 54, 401

Pacini, F. 1968, Nature, 219, 145

Pen, U.-L., \& Connor, L. 2015, ApJ, 807, 179

Petroff, E., van Straten, W., Johnston, S., et al. 2014, ApJl, 789, LL26

Petroff, E., Bailes, M., Barr, E. D., et al. 2015, MNRAS, 447, 246

Petroff, E. et al. 2015, arXiv:1504.02165

Petroff, E., Johnston, S., Keane, E. F., et al. 2015, MNRAS, 454, 457

Petroff, E., Barr, E. D., Jameson, A., et al. 2016, PASA, 33, e045

Petroff, E., Burke-Spolaor, S., Keane, E. F., et al. 2017, arXiv:1705.02911

Pietka, M., Fender, R. P., \& Keane, E. F. 2015, MNRAS, 446, 3687

Piro, A. L. 2012, ApJ, 755, 80

Popov, S. B., \& Postnov, K. A. 2013, arXiv:1307.4924

Press, W. H., Teukolsky, S. A., Vetterling, W. T., \& Flannery, B. P. 1992, Cambridge: University Press, c1992, 2nd ed.

Rajwade, K. M., \& Lorimer, D. R. 2017, MNRAS, 465, 2286

Ravi, V., Shannon, R. M., \& Jameson, A. 2014, arXiv:1412.1599 
Ravi, V., Shannon, R. M., Bailes, M., et al. 2016, Science, 354, 1249

Roeder, R. C., \& Verreault, R. T. 1969, ApJ, 155, 1047

Rickett, B. J. 1969, Nature, 221, 158

Ritchings, R. T. 1976, MNRAS, 176, 249

Rubin, D., \& Loeb, A. 2014, Journal of Cosmology and Astroparticle Physics, 1, 051

Saint-Hilaire, P., Benz, A. O., \& Monstein, C. 2014, ApJ, 795, 19

Scheuer, P. A. G. 1968, Nature, 218, 920

Scholz, P., Spitler, L. G., Hessels, J. W. T., et al. 2016, arXiv:1603.08880

Shannon, R. M., \& Ravi, V. 2017, ApJL, 837, L22

Shull, J. M., Smith, B. D., \& Danforth, C. W. 2012, ApJ, 759, 23

Sieber, W. 1982, A\&A, 113, 311

Spitler, L. G., Cordes, J. M., Hessels, J. W. T., et al. 2014, ApJ, 790, 101

Spitler, L. G., Scholz, P., Hessels, J. W. T., et al. 2016, Nature, 531, 202

Staelin, D. H., \& Reifenstein, E. C., III 1968, Science, 162, 1481

Stanek, R., Evrard, A. E., Böhringer, H., Schuecker, P., \& Nord, B. 2006, ApJ, 648, 956

Staveley-Smith, L., Wilson, W. E., Bird, T. S., et al. 1996, PASA, 13, 243 
Stephenson, F. R., \& Green, D. A. 2002, Historical supernovae and their remnants, by F. Richard Stephenson and David A. Green. International series in astronomy and astrophysics, vol. 5. Oxford: Clarendon Press, 2002, ISBN 0198507666, 5.

Soglasnov, V. A., Popov, M. V., Bartel, N., et al. 2004, ApJ, 616, 439

Tendulkar, S. P., Kaspi, V. M., \& Patel, C. 2016, ApJ, 827, 59

Tendulkar, S. P., Bassa, C. G., Cordes, J. M., et al. 2017, ApJL, 834, L7

Thornton, D., Stappers, B., Bailes, M., et al. 2013, Science, 341, 53

Thornton D. P. G., 2013, PhD thesis, The University of Manchester

Totani, T. 2013, Publications of the Astronomical Society of Japan, 65, L12

Vedantham, H. K., Ravi, V., Mooley, K., et al. 2016, ApJL, 824, L9

Williams, P. K. G., \& Berger, E. 2016, ApJL, 821, L22

Wall, J. V., Jenkins, C. R., Ellis, R., et al. 2003, Practical statistics for astronomers, by J.V. Wall and C.R. Jenkins. Cambridge observing handbooks for research astronomers, vol. 3. Cambridge, UK: Cambridge University Press, 2003

Press, W. H., Teukolsky, S. A., Vetterling, W. T., \& Flannery, B. P. 1992, 2nd ed., Cambridge: University Press, —c1992

Wolszczan, A., Cordes, J., \& Stinebring, D. 1984, Birth and Evolution of Neutron Stars: Issues Raised by Millisecond Pulsars, 63 
Wang, J.-S., Yang, Y.-P., Wu, X.-F., Dai, Z.-G., \& Wang, F.-Y. 2016, ApJL, 822, L7

Wei, J.-J., Gao, H., Wu, X.-F., \& Mészáros, P. 2015, Physical Review Letters, 115, 261101

Weiler, K. W. 1983, The Observatory, 103, 85

Weltevrede, P., Abdo, A. A., Ackermann, M., et al. 2010, ApJ, 708, 1426

Xu, J., \& Han, J. L. 2015, Research in Astronomy and Astrophysics, 15, 1629

Xu, S., \& Zhang, B. 2016, ApJ, 832, 199

Yao, J. M., Manchester, R. N., \& Wang, N. 2016, arXiv:1610.09448

Yang, Y.-P., \& Zhang, B. 2016, ApJL, 830, L31

Zarka, P. 1998, , 103, 20159

Zarka, P., Treumann, R. A., Ryabov, B. P., \& Ryabov, V. B. 2001, Ap\&SS, 277, 293

Zheng, Z., Ofek, E. O., Kulkarni, S. R., Neill, J. D., \& Juric, M. 2014, ApJ, 797, 71

Zhang, B. 2016, ApJL, 822, L14

Zhou, B., Li, X., Wang, T., Fan, Y.-Z., \& Wei, D.-M. 2014, Phys. Rev. D, 89, 107303 